\title{
VECTOR DISSIPATIVITY THEORY FOR LARGE-SCALE IMPULSIVE DYNAMICAL SYSTEMS
}

\author{
WASSIM M. HADDAD, VIJAYSEKHAR CHELLABOINA, \\ QING HUI, AND SERGEY NERSESOV
}

Received 17 October 2003 and in revised form 30 March 2004

\begin{abstract}
Modern complex large-scale impulsive systems involve multiple modes of operation placing stringent demands on controller analysis of increasing complexity. In analyzing these large-scale systems, it is often desirable to treat the overall impulsive system as a collection of interconnected impulsive subsystems. Solution properties of the large-scale impulsive system are then deduced from the solution properties of the individual impulsive subsystems and the nature of the impulsive system interconnections. In this paper, we develop vector dissipativity theory for large-scale impulsive dynamical systems. Specifically, using vector storage functions and vector hybrid supply rates, dissipativity properties of the composite large-scale impulsive systems are shown to be determined from the dissipativity properties of the impulsive subsystems and their interconnections. Furthermore, extended Kalman-Yakubovich-Popov conditions, in terms of the impulsive subsystem dynamics and interconnection constraints, characterizing vector dissipativeness via vector system storage functions, are derived. Finally, these results are used to develop feedback interconnection stability results for large-scale impulsive dynamical systems using vector Lyapunov functions.
\end{abstract}

\section{Introduction}

Recent technological demands have required the analysis and control design of increasingly complex, large-scale nonlinear dynamical systems. The complexity of modern controlled large-scale dynamical systems is further exacerbated by the use of hierarchial embedded control subsystems within the feedback control system, that is, abstract decisionmaking units performing logical checks that identify system mode operation and specify the continuous-variable subcontroller to be activated. Such systems typically possess a multiechelon hierarchical hybrid decentralized control architecture characterized by continuous-time dynamics at the lower levels of the hierarchy and discrete-time dynamics at the higher levels of the hierarchy (see $[1,25]$ and the numerous references therein). 
The lower-level units directly interact with the dynamical system to be controlled while the higher-level units receive information from the lower-level units as inputs and provide (possibly discrete) output commands which serve to coordinate and reconcile the (sometimes competing) actions of the lower-level units. The hierarchical controller organization reduces processor cost and controller complexity by breaking up the processing task into relatively small pieces and decomposing the fast and the slow control functions. Typically, the higher-level units perform logical checks that determine system mode operation, while the lower-level units execute continuous-variable commands for a given system mode of operation.

In analyzing hybrid large-scale dynamical systems, it is often desirable to treat the overall system as a collection of interconnected subsystems. The behavior of the composite hybrid large-scale system can then be predicted from the behaviors of the individual subsystems and their interconnections. The mathematical description of many of these systems can be characterized by impulsive differential equations $[10,15]$. In particular, general hybrid dynamical systems involve an abstract axiomatic definition of a dynamical system involving left-continuous (or right-continuous) flows defined on a completely ordered time set as a mapping between vector spaces satisfying an appropriate set of axioms, and include hybrid inputs and hybrid outputs that take their values in appropriate vector spaces $[8,22,28]$. In contrast, impulsive dynamical systems are a subclass of hybrid dynamical systems and consist of three elements, namely, a continuous-time differential equation, which governs the motion of the dynamical system between impulsive events; a difference equation, which governs the way that the system states are instantaneously changed when an impulsive event occurs; and a criterion for determining when the states are to be reset $[10,15]$.

An approach to analyzing large-scale dynamical systems was introduced by the pioneering work of Šiljak [26] and involves the notion of connective stability. In particular, the large-scale dynamical system is decomposed into a collection of subsystems with local dynamics and uncertain interactions. Then, each subsystem is considered independently so that the stability of each subsystem is combined with the interconnection constraints to obtain a vector Lyapunov function for the composite large-scale dynamical system guaranteeing connective stability for the overall system. Vector Lyapunov functions were first introduced by Bellman [2] and Matrosov [21] and further developed in $[7,16,17,18,19,20,23,27]$, with [7, 17, 26, 27] exploiting their utility for analyzing large-scale systems. Extensions of vector Lyapunov function theory that include matrixvalued Lyapunov functions for stability analysis of large-scale dynamical systems appear in the monographs by Martynyuk $[19,20]$. The use of vector Lyapunov functions in largescale system analysis offers a very flexible framework since each component of the vector Lyapunov function can satisfy less rigid requirements as compared to a single scalar Lyapunov function. Weakening the hypothesis on the Lyapunov function enlarges the class of Lyapunov functions that can be used for analyzing the stability of large-scale dynamical systems. In particular, each component of a vector Lyapunov function need not be positive-definite with a negative or even negative-semidefinite derivative. The time derivative of the vector Lyapunov function need only satisfy an element-by-element vector inequality involving a vector field of a certain comparison system. 
In light of the facts that energy flow modeling arises naturally in large-scale dynamical systems and that vector Lyapunov functions provide a powerful stability analysis framework for these systems, it seems natural that hybrid dissipativity theory $[8,10,11]$, on the subsystem level, should play a key role in analyzing large-scale impulsive dynamical systems. Specifically, hybrid dissipativity theory provides a fundamental framework for the analysis and design of impulsive dynamical systems using an input-output description based on system energy related considerations (here the notion of energy refers to abstract energy for which a physical system energy interpretation is not necessary) $[8,10]$. The hybrid dissipation hypothesis on impulsive dynamical systems results in a fundamental constraint on their dynamic behavior wherein a dissipative impulsive dynamical system can only deliver a fraction of its energy to its surroundings and can only store a fraction of the work done to it. Such conservation laws are prevalent in large-scale impulsive dynamical systems such as aerospace systems, power systems, network systems, telecommunication systems, and transportation systems. Since these systems have numerous input-output properties related to conservation, dissipation, and transport of energy, extending hybrid dissipativity theory to capture conservation and dissipation notions on the subsystem level would provide a natural energy flow model for large-scale impulsive dynamical systems. Aggregating the dissipativity properties of each of the impulsive subsystems by appropriate storage functions and hybrid supply rates would allow us to study the dissipativity properties of the composite large-scale impulsive system using vector storage functions and vector hybrid supply rates. Furthermore, since vector Lyapunov functions can be viewed as generalizations of composite energy functions for all of the impulsive subsystems, a generalized notion of hybrid dissipativity, namely, vector hybrid dissipativity, with appropriate vector storage functions and vector hybrid supply rates, can be used to construct vector Lyapunov functions for nonlinear feedback large-scale impulsive systems by appropriately combining vector storage functions for the forward and feedback large-scale impulsive systems. Finally, as in classical dynamical system theory, vector dissipativity theory can play a fundamental role in addressing robustness, disturbance rejection, stability of feedback interconnections, and optimality for large-scale impulsive dynamical systems.

In this paper, we develop vector dissipativity notions for large-scale nonlinear impulsive dynamical systems, a notion not previously considered in the literature. In particular, we introduce a generalized definition of dissipativity for large-scale nonlinear impulsive dynamical systems in terms of a hybrid vector inequality involving a vector hybrid supply rate, a vector storage function, and an essentially nonnegative, semistable dissipation matrix. Generalized notions of vector available storage and vector required supply are also defined and shown to be element-by-element ordered, nonnegative, and finite. On the impulsive subsystem level, the proposed approach provides an energy flow balance over the continuous-time dynamics and the resetting events in terms of the stored subsystem energy, the supplied subsystem energy, the subsystem energy gained from all other subsystems independent of the subsystem coupling strengths, and the subsystem energy dissipated. Furthermore, for large-scale impulsive dynamical systems decomposed into interconnected impulsive subsystems, dissipativity of the composite impulsive system is shown to be determined from the dissipativity properties of the individual impulsive 
subsystems and the nature of the interconnections. In addition, we develop extended Kalman-Yakubovich-Popov conditions, in terms of the local impulsive subsystem dynamics and the interconnection constraints, for characterizing vector dissipativeness via vector storage functions for large-scale impulsive dynamical systems. Using the concepts of vector dissipativity and vector storage functions as candidate vector Lyapunov functions, we develop feedback interconnection stability results of large-scale impulsive nonlinear dynamical systems. General stability criteria are given for Lyapunov and asymptotic stability of feedback large-scale impulsive dynamical systems. In the case of vector quadratic supply rates involving net subsystem powers and input-output subsystem energies, these results provide a positivity and small gain theorem for large-scale impulsive systems predicated on vector Lyapunov functions. Finally, it is important to note that vector dissipativity notions were first addressed in [13] in the context of continuoustime, large-scale dynamical systems. However, the results of [13] predominately concentrate on connections between thermodynamic models and large-scale dynamical systems. Kalman-Yakubovich-Popov conditions characterizing vector dissipativeness via vector system storage functions and feedback interconnection stability results for large-scale systems are not addressed in [13].

\section{Mathematical preliminaries}

In this section, we introduce notations, several definitions, and some key results needed for analyzing large-scale impulsive dynamical systems. Let $\mathbb{R}$ denote the set of real numbers, $\overline{\mathbb{Z}}_{+}$the set of nonnegative integers, $\mathbb{R}^{n}$ the set of $n \times 1$ column vectors, $\mathbb{S}^{n}$ the set of $n \times n$ symmetric matrices, $\mathbb{N}^{n}$ (resp., $\mathbb{P}^{n}$ ) the set of $n \times n$ nonnegative- (resp., positive-) definite matrices, $(\cdot)^{\mathrm{T}}$ transpose, and $I_{n}$ or $I$ the $n \times n$ identity matrix. For $v \in \mathbb{R}^{q}$, we write $v \geq \geq 0$ (resp., $v \gg 0$ ) to indicate that every component of $v$ is nonnegative (resp., positive). In this case, we say that $v$ is nonnegative or positive, respectively. Let $\overline{\mathbb{R}}_{+}^{q}$ and $\mathbb{R}_{+}^{q}$ denote the nonnegative and positive orthants of $\mathbb{R}^{q}$; that is, if $v \in \mathbb{R} q$, then $v \in \overline{\mathbb{R}}_{+}^{q}$ and $v \in \mathbb{R}_{+}^{q}$ are equivalent, respectively, to $v \geq \geq 0$ and $v \gg 0$. Finally, we write $\|\cdot\|$ for the Euclidean vector norm, $V^{\prime}(x)$ for the Fréchet derivative of $V$ at $x, \mathscr{B}_{\varepsilon}(\alpha), \alpha \in \mathbb{R}^{n}, \varepsilon>0$, for the open ball centered at $\alpha$ with radius $\varepsilon$, and $M \geq 0$ (resp., $M>0$ ) to denote the fact that the Hermitian matrix $M$ is nonnegative- (resp., positive-) definite. The following definition introduces the notion of essentially nonnegative and nonnegative matrices.

Definition $2.1[3,5,9]$. Let $W \in \mathbb{R}^{q \times q}$. $W$ is essentially nonnegative if $W_{(i, j)} \geq 0, i, j=$ $1, \ldots, q, i \neq j$, where $W_{(i, j)}$ denotes the $(i, j)$ th entry of $W . W$ is nonnegative (resp., positive) if $W_{(i, j)} \geq 0$ (resp., $\left.W_{(i, j)}>0\right), i, j=1, \ldots, q$.

The following definition introduces the notion of class $\mathcal{W}$ functions involving quasimonotone increasing functions.

Definition 2.2 [26]. A function $w=\left[w_{1}, \ldots, w_{q}\right]^{\mathrm{T}}: \mathbb{R}^{q} \rightarrow \mathbb{R}^{q}$ is of class $\mathcal{W}$ if $w_{i}\left(r^{\prime}\right) \leq w_{i}\left(r^{\prime \prime}\right)$, $i=1, \ldots, q$, for all $r^{\prime}, r^{\prime \prime} \in \mathbb{R}^{q}$ such that $r_{j}^{\prime} \leq r_{j}^{\prime \prime}, r_{i}^{\prime}=r_{i}^{\prime \prime}, j=1, \ldots, q, i \neq j$, where $r_{i}$ denotes the ith component of $r$.

If $w(\cdot) \in \mathcal{W}$, we say that $w$ satisfies the Kamke condition. Note that if $w(r)=W r$, where $W \in \mathbb{R}^{q \times q}$, then the function $w(\cdot)$ is of class $\mathcal{W}$ if and only if $W$ is essentially 
nonnegative. Furthermore, note that it follows from Definition 2.2 that any scalar $(q=1)$ function $w(r)$ is of class $\mathcal{W}$. The following definition introduces the notion of essentially nonnegative functions $[4,9]$.

Definition 2.3. Let $w=\left[w_{1}, \ldots, w_{q}\right]^{\mathrm{T}}: \mathscr{V} \rightarrow \mathbb{R}^{q}$, where $\mathscr{V}$ is an open subset of $\mathbb{R}^{q}$ that contains $\overline{\mathbb{R}}_{+}^{q}$. Then $w$ is essentially nonnegative if $w_{i}(r) \geq 0$ for all $i=1, \ldots, q$ and $r \in \overline{\mathbb{R}}_{+}^{q}$ such that $r_{i}=0$.

Note that if $w: \mathbb{R}^{q} \rightarrow \mathbb{R}^{q}$ is such that $w(\cdot) \in \mathcal{W}$ and $w(0) \geq \geq 0$, then $w$ is essentially nonnegative; the converse however is not generally true. However, if $w(r)=W r$, where $W \in \mathbb{R}^{q \times q}$ is essentially nonnegative, then $w(\cdot)$ is essentially nonnegative and $w(\cdot) \in \mathcal{W}$.

Proposition $2.4[4,9]$. Suppose $\overline{\mathbb{R}}_{+}^{q} \subset \mathcal{V}$. Then $\overline{\mathbb{R}}_{+}^{q}$ is an invariant set with respect to

$$
\dot{r}(t)=w(r(t)), \quad r(0)=r_{0}, t \geq t_{0},
$$

where $r_{0} \in \overline{\mathbb{R}}_{+}^{q}$, if and only if $w: \mathscr{V} \rightarrow \mathbb{R}^{q}$ is essentially nonnegative.

The following corollary to Proposition 2.4 is immediate.

Corollary 2.5. Let $W \in \mathbb{R}^{q \times q}$. Then $W$ is essentially nonnegative if and only if $e^{W t}$ is nonnegative for all $t \geq 0$.

It follows from Proposition 2.4 that if $r_{0} \geq \geq 0$, then $r(t) \geq \geq 0, t \geq t_{0}$, if and only if $w(\cdot)$ is essentially nonnegative. In this case, the usual stability definitions for the equilibrium solution $r(t) \equiv r_{\mathrm{e}}$ to (2.1) are not valid. In particular, stability notions need to be defined with respect to relatively open subsets of $\overline{\mathbb{R}}_{+}^{q}$ containing $r_{\mathrm{e}}[12,13]$. The following lemma is needed for developing several of the results in later sections. For the statement of this lemma, recall that a matrix $W \in \mathbb{R}^{q \times q}$ is semistable if and only if $\lim _{t \rightarrow \infty} e^{W t}$ exists $[5,9]$, while $W$ is asymptotically stable if and only if $\lim _{t \rightarrow \infty} e^{W t}=0$.

Lemma 2.6 [12]. Suppose $W \in \mathbb{R}^{q \times q}$ is essentially nonnegative. If $W$ is semistable (resp., asymptotically stable), then there exist a scalar $\alpha \geq 0$ (resp., $\alpha>0$ ) and a nonnegative vector $p \in \overline{\mathbb{R}}_{+}^{q}, p \neq 0$, (resp., positive vector $p \in \mathbb{R}_{+}^{q}$ ) such that

$$
W^{\mathrm{T}} p+\alpha p=0
$$

Next, we present a stability result for large-scale impulsive dynamical systems using vector Lyapunov functions. In particular, we consider state-dependent impulsive dynamical systems of the form

$$
\begin{gathered}
\dot{x}(t)=F_{\mathrm{c}}(x(t)), \quad x\left(t_{0}\right)=x_{0}, x(t) \notin \mathscr{E}_{x}, t \geq t_{0}, \\
\Delta x(t)=F_{\mathrm{d}}(x(t)), \quad x(t) \in \mathscr{E}_{x},
\end{gathered}
$$

where $x(t) \in \mathscr{D}, \mathscr{D} \subseteq \mathbb{R}^{n}$ is an open set with $0 \in \mathscr{D}, \Delta x(t) \triangleq x\left(t^{+}\right)-x(t), F_{\mathrm{c}}: \mathscr{D} \rightarrow \mathbb{R}^{n}$ is Lipschitz continuous and satisfies $F_{\mathrm{c}}(0)=0, F_{\mathrm{d}}: \mathscr{D} \rightarrow \mathbb{R}^{n}$ is continuous, and $\mathscr{L}_{x} \subset$ $\mathscr{D} \subseteq \mathbb{R}^{n}$ is a resetting set. Here, we assume that (2.3) and (2.4) characterize a large-scale impulsive dynamical system composed of $q$ interconnected subsystems such that, for all $i=1, \ldots, q$, each element of $F_{\mathrm{c}}(x)$ and $F_{\mathrm{d}}(x)$ is given by $F_{\mathrm{c} i}(x)=f_{\mathrm{c} i}\left(x_{i}\right)+\Phi_{\mathrm{c} i}(x)$ and 
$F_{\mathrm{d} i}(x)=f_{\mathrm{d} i}\left(x_{i}\right)+\mathscr{I}_{\mathrm{d} i}(x)$, respectively, where $f_{\mathrm{c} i}: \mathscr{D}_{i} \subseteq \mathbb{R}^{n_{i}} \rightarrow \mathbb{R}^{n_{i}}$ and $f_{\mathrm{d} i}: \mathscr{D}_{i} \subseteq \mathbb{R}^{n_{i}} \rightarrow \mathbb{R}^{n_{i}}$ define the vector fields of each isolated impulsive subsystem of (2.3) and (2.4), $\mathscr{I}_{\mathrm{c} i}: \mathscr{D} \rightarrow$ $\mathbb{R}^{n_{i}}$ and $\mathscr{I}_{\mathrm{d} i}: \mathscr{D} \rightarrow \mathbb{R}^{n_{i}}$ define the structure of interconnection dynamics of the $i$ th impulsive subsystem with all other impulsive subsystems, $x_{i} \in \mathscr{D}_{i} \subseteq \mathbb{R}^{n_{i}}, f_{\mathrm{ci}}(0)=0, \mathscr{\Phi}_{\mathrm{ci}}(0)=0$, and $\sum_{i=1}^{q} n_{i}=n$. For the large-scale impulsive dynamical system (2.3), (2.4), we note that the subsystem states that $x_{i}(t), t \geq t_{0}$, for all $i=1, \ldots, q$, belong to $\mathscr{D}_{i} \subseteq \mathbb{R}^{n_{i}}$ as long as $x(t) \triangleq\left[x_{1}^{\mathrm{T}}(t), \ldots, x_{q}^{\mathrm{T}}(t)\right]^{\mathrm{T}} \in \mathscr{D}, t \geq t_{0}$. We make the following additional assumptions:

(A1) if $x(t) \in \overline{\mathscr{E}}_{x} \backslash \mathscr{E}_{x}$, then there exists $\varepsilon>0$ such that, for all $0<\delta<\varepsilon, x(t+\delta) \notin \mathscr{E}_{x}$;

(A2) if $x \in \mathscr{E}_{x}$, then $x+F_{\mathrm{d}}(x) \notin \mathscr{L}_{x}$.

Assumption (A1) ensures that if a trajectory reaches the closure of $\mathscr{L}_{x}$ at a point that does not belong to $\mathscr{E}_{x}$, then the trajectory must be directed away from $\mathscr{E}_{x}$; that is, a trajectory cannot enter $\mathscr{E}_{x}$ through a point that belongs to the closure of $\mathscr{E}_{x}$ but not to $\mathscr{E}_{x}$. Furthermore, (A2) ensures that when a trajectory intersects the resetting set $\mathscr{E}_{x}$, it instantaneously exits $\mathscr{E}_{x}$. Finally, we note that if $x_{0} \in \mathscr{L}_{x}$, then the system initially resets to $x_{0}^{+}=x_{0}+F_{\mathrm{d}}\left(x_{0}\right) \notin \mathscr{E}_{x}$, which serves as the initial condition for the continuous dynamics (2.3). It follows from (A1) and (A2) that $\partial \mathscr{E}_{x} \cap \mathscr{E}_{x}$ is closed and hence the resetting times $\tau_{k}\left(x_{0}\right)$ are well defined and distinct. Furthermore, it follows from (A2) that if $x^{*} \in \mathbb{R}^{n}$ satisfies $F_{\mathrm{d}}\left(x^{*}\right)=0$, then $x^{*} \notin \mathscr{E}_{x}$. To see this, suppose $x^{*} \in \mathscr{E}_{x}$. Then $x^{*}+F_{\mathrm{d}}\left(x^{*}\right)=$ $x^{*} \in \mathscr{L}_{x}$, contradicting (A2). In particular, we note that $0 \notin \mathscr{E}_{x}$. For further insights on assumptions (A1) and (A2), the interested reader is referred to $[8,10]$.

The next theorem presents a stability result for (2.3), (2.4) via vector Lyapunov functions by relating the stability properties of a comparison system to the stability properties of the large-scale impulsive dynamical system.

Theorem $2.7[15,24]$. Consider the large-scale impulsive dynamical system given by (2.3), (2.4). Suppose there exist a continuously differentiable vector function $V: \mathscr{D} \rightarrow \overline{\mathbb{R}}_{+}^{q}$ and a positive vector $p \in \mathbb{R}_{+}^{q}$ such that $V(0)=0$, the scalar function $v: \mathscr{D} \rightarrow \overline{\mathbb{R}}_{+}$defined by $v(x)=$ $p^{\mathrm{T}} V(x), x \in \mathscr{D}$, is such that $v(0)=0, v(x)>0, x \neq 0$, and

$$
\begin{gathered}
V^{\prime}(x) F_{\mathrm{c}}(x) \leq \leq w_{\mathrm{c}}(V(x)), \quad x \notin \mathscr{E}_{x}, \\
V\left(x+F_{\mathrm{d}}(x)\right) \leq \leq V(x), \quad x \in \mathscr{E}_{x},
\end{gathered}
$$

where $w_{\mathrm{c}}: \overline{\mathbb{R}}_{+}^{q} \rightarrow \mathbb{R}^{q}$ is a class W function such that $w_{\mathrm{c}}(0)=0$. Then the stability properties of the zero solution $r(t) \equiv 0$ to

$$
\dot{r}(t)=w_{\mathrm{c}}(r(t)), \quad r\left(t_{0}\right)=r_{0}, \quad t \geq t_{0},
$$

imply the corresponding stability properties of the zero solution $x(t) \equiv 0$ to (2.3), (2.4). That is, if the zero solution $r(t) \equiv 0$ to (2.6) is Lyapunov (resp., asymptotically) stable, then the zero solution $x(t) \equiv 0$ to (2.3), (2.4) is Lyapunov (resp., asymptotically) stable. If, in addition, $\mathscr{D}=\mathbb{R}^{n}$ and $V(x) \rightarrow \infty$ as $\|x\| \rightarrow \infty$, then global asymptotic stability of the zero solution $r(t) \equiv 0$ to (2.6) implies global asymptotic stability of the zero solution $x(t) \equiv 0$ to (2.3), (2.4). 
If $V: \mathscr{D} \rightarrow \overline{\mathbb{R}}_{+}^{q}$ satisfies the conditions of Theorem 2.7 , we say that $V(x), x \in \mathscr{D}$, is a vector Lyapunov function for the large-scale impulsive dynamical system (2.3), (2.4). Finally, we recall the standard notions of dissipativity and exponential dissipativity $[8,10]$ for input/state-dependent impulsive dynamical systems $\mathscr{G}$ of the form

$$
\begin{gathered}
\dot{x}(t)=f_{\mathrm{c}}(x(t))+G_{\mathrm{c}}(x(t)) u_{\mathrm{c}}(t), \quad x\left(t_{0}\right)=x_{0}, \quad\left(x(t), u_{\mathrm{c}}(t)\right) \notin \mathscr{L}, \\
\Delta x(t)=f_{\mathrm{d}}(x(t))+G_{\mathrm{d}}(x(t)) u_{\mathrm{d}}(t), \quad\left(x(t), u_{\mathrm{c}}(t)\right) \in \mathscr{L}, \\
y_{\mathrm{c}}(t)=h_{\mathrm{c}}(x(t))+J_{\mathrm{c}}(x(t)) u_{\mathrm{c}}(t), \quad\left(x(t), u_{\mathrm{c}}(t)\right) \notin \mathscr{L}, \\
y_{\mathrm{d}}(t)=h_{\mathrm{d}}(x(t))+J_{\mathrm{d}}(x(t)) u_{\mathrm{d}}(t), \quad\left(x(t), u_{\mathrm{c}}(t)\right) \in \mathscr{L},
\end{gathered}
$$

where $t \geq t_{0}, x(t) \in \mathscr{D} \subseteq \mathbb{R}^{n}, u_{\mathrm{c}}(t) \in \boldsymbol{U}_{\mathrm{c}} \subseteq \mathbb{R}^{m_{\mathrm{c}}}, u_{\mathrm{d}}\left(t_{k}\right) \in \boldsymbol{U}_{\mathrm{d}} \subseteq \mathbb{R}^{m_{\mathrm{d}}}, t_{k}$ denotes the $k$ th instant of time at which $\left(x(t), u_{\mathrm{c}}(t)\right)$ intersects $\mathscr{E} \subset \mathscr{D} \times \boldsymbol{U}_{\mathrm{c}}$ for a particular trajectory $x(t)$ and input $u_{\mathrm{c}}(t), y_{\mathrm{c}}(t) \in \mathscr{Y}_{\mathrm{c}} \subseteq \mathbb{R}^{l_{\mathrm{c}}}, y_{\mathrm{d}}\left(t_{k}\right) \in \mathscr{Y}_{\mathrm{d}} \subseteq \mathbb{R}^{l_{\mathrm{d}}}, f_{\mathrm{c}}: \mathscr{D} \rightarrow \mathbb{R}^{n}$ is Lipschitz continuous and satisfies $f_{\mathrm{c}}(0)=0, G_{\mathrm{c}}: \mathscr{D} \rightarrow \mathbb{R}^{n \times m_{\mathrm{c}}}, f_{\mathrm{d}}: \mathscr{D} \rightarrow \mathbb{R}^{n}$ is continuous, $G_{\mathrm{d}}: \mathscr{D} \rightarrow \mathbb{R}^{n \times m_{\mathrm{d}}}$, $h_{\mathrm{c}}: \mathscr{D} \rightarrow \mathbb{R}^{l_{\mathrm{c}}}$ satisfies $h_{\mathrm{c}}(0)=0, J_{\mathrm{c}}: \mathscr{D} \rightarrow \mathbb{R}^{l_{\mathrm{c}} \times m_{\mathrm{c}}}, h_{\mathrm{d}}: \mathscr{D} \rightarrow \mathbb{R}^{l_{\mathrm{d}}}$, and $J_{\mathrm{d}}: \mathscr{D} \rightarrow \mathbb{R}^{l_{\mathrm{d}} \times m_{\mathrm{d}}}$. For the impulsive dynamical system $\mathscr{G}$, we assume that the required properties for the existence and uniqueness of solutions are satisfied; that is, $u_{\mathrm{c}}(\cdot)$ satisfies sufficient regularity conditions such that (2.7) has a unique solution forward in time. For the impulsive dynamical system $\mathcal{G}_{\text {given by }}(2.7),(2.8),(2.9)$, and (2.10), a function $\left(s_{\mathrm{c}}\left(u_{\mathrm{c}}, y_{\mathrm{c}}\right), s_{\mathrm{d}}\left(u_{\mathrm{d}}, y_{\mathrm{d}}\right)\right)$, where $s_{\mathrm{c}}: \boldsymbol{U}_{\mathrm{c}} \times \mathscr{y}_{\mathrm{c}} \rightarrow \mathbb{R}$ and $s_{\mathrm{d}}: \boldsymbol{U}_{\mathrm{d}} \times \mathscr{y}_{\mathrm{d}} \rightarrow \mathbb{R}$ are such that $s_{\mathrm{c}}(0,0)=0$ and $s_{\mathrm{d}}(0,0)=0$, is called a hybrid supply rate $[8,10]$ if it is locally integrable for all input-output pairs satisfying (2.7), (2.9); that is, for all input-output pairs $u_{\mathrm{c}} \in \mathcal{U}_{\mathrm{c}}, y_{\mathrm{c}} \in \mathscr{Y}_{\mathrm{c}}$ satisfying (2.7), (2.9), $s_{\mathrm{c}}(\cdot, \cdot)$ satisfies $\int_{t}^{\hat{t}}\left|s_{\mathrm{c}}\left(u_{\mathrm{c}}(\sigma), y_{\mathrm{c}}(\sigma)\right)\right| \mathrm{d} \sigma<\infty, t, \hat{t} \geq 0$. Note that since all input-output pairs $u_{\mathrm{d}}\left(t_{k}\right) \in \boldsymbol{U}_{\mathrm{d}}, y_{\mathrm{d}}\left(t_{k}\right) \in \mathscr{Y}_{\mathrm{d}}$ satisfying (2.8), (2.10) are defined for discrete instants, $s_{\mathrm{d}}(\cdot, \cdot)$

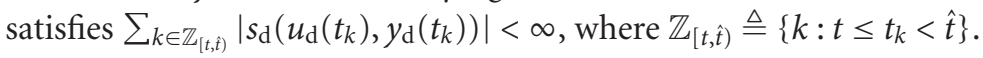

Definition 2.8 [10]. The impulsive dynamical system $\mathscr{G}$ given by (2.7), (2.8), (2.9), and (2.10) is exponentially dissipative (resp., dissipative) with respect to the hybrid supply rate $\left(s_{\mathrm{c}}, s_{\mathrm{d}}\right)$ if there exist a continuous, nonnegative-definite function $v_{\mathrm{s}}: \mathscr{D} \rightarrow \mathbb{R}$ and a scalar $\varepsilon>0$ (resp., $\varepsilon=0$ ) such that $v_{s}(0)=0$, called a storage function, and the hybrid dissipation inequality

$$
\begin{aligned}
e^{\varepsilon T} v_{\mathrm{s}}(x(T)) \leq & e^{\varepsilon t_{0}} v_{\mathrm{s}}\left(x\left(t_{0}\right)\right)+\int_{t_{0}}^{T} e^{\varepsilon t} s_{\mathrm{c}}\left(u_{\mathrm{c}}(t), y_{\mathrm{c}}(t)\right) \mathrm{d} t \\
& +\sum_{k \in \mathbb{Z}_{\left[t_{0}, T\right)}} e^{\varepsilon t_{k}} s_{\mathrm{d}}\left(u_{\mathrm{d}}\left(t_{k}\right), y_{\mathrm{d}}\left(t_{k}\right)\right), \quad T \geq t_{0},
\end{aligned}
$$

is satisfied for all $T \geq t_{0}$. The impulsive dynamical system $\mathscr{G}$ given by (2.7), (2.8), (2.9), and $(2.10)$ is lossless with respect to the hybrid supply rate $\left(s_{\mathrm{c}}, s_{\mathrm{d}}\right)$ if the hybrid dissipation inequality is satisfied as an equality with $\varepsilon=0$ for all $T \geq t_{0}$.

The following result gives necessary and sufficient conditions for dissipativity over an interval $t \in\left(t_{k}, t_{k+1}\right]$ involving the consecutive resetting times $t_{k}$ and $t_{k+1}$. First, however, the following definition is required. 
Definition 2.9 [10]. A large-scale impulsive dynamical system $\mathscr{G}$ given by (2.7), (2.8), (2.9), and (2.10) is completely reachable if for all $\left(t_{0}, x_{\mathrm{i}}\right) \in \mathbb{R} \times \mathscr{D}$, there exist a finite time $t_{\mathrm{i}}<t_{0}$, a square integrable input $u_{\mathrm{c}}(t)$ defined on $\left[t_{\mathrm{i}}, t_{0}\right]$, and inputs $u_{\mathrm{d}}\left(t_{k}\right)$ defined on $k \in \mathbb{Z}_{\left[\mathrm{t}_{\mathrm{i}}, t_{0}\right)}$ such that the state $x(t), t \geq t_{\mathrm{i}}$, can be driven from $x\left(t_{\mathrm{i}}\right)=0$ to $x\left(t_{0}\right)=x_{\mathrm{i}}$.

Theorem 2.10 [10]. Assume $G$ is completely reachable. Then $G$ is exponentially dissipative (resp., dissipative) with respect to the hybrid supply rate $\left(s_{\mathrm{c}}, s_{\mathrm{d}}\right)$ if and only if there exist a continuous nonnegative-definite function $v_{\mathrm{s}}: \mathscr{D} \rightarrow \mathbb{R}$ and a scalar $\varepsilon>0$ (resp., $\varepsilon=0$ ) such that $v_{\mathrm{s}}(0)=0$ and for all $k \in \overline{\mathbb{Z}}_{+}$,

$$
\begin{gathered}
e^{\varepsilon \hat{t}} v_{\mathrm{s}}(x(\hat{t})) \leq e^{\varepsilon t} v_{\mathrm{s}}(x(t))+\int_{t}^{\hat{t}} e^{\varepsilon s} s_{\mathrm{c}}\left(u_{\mathrm{c}}(s), y_{\mathrm{c}}(s)\right) \mathrm{d} s, \quad t_{k}<t \leq \hat{t} \leq t_{k+1}, \\
v_{\mathrm{s}}\left(x\left(t_{k}\right)+f_{\mathrm{d}}\left(x\left(t_{k}\right)\right)+G_{\mathrm{d}}\left(x\left(t_{k}\right)\right) u_{\mathrm{d}}\left(t_{k}\right)\right) \leq v_{\mathrm{s}}\left(x\left(t_{k}\right)\right)+s_{\mathrm{d}}\left(u_{\mathrm{d}}\left(t_{k}\right), y_{\mathrm{d}}\left(t_{k}\right)\right) .
\end{gathered}
$$

Finally, $G$ given by (2.7), (2.8), (2.9), and (2.10) is lossless with respect to the hybrid supply rate $\left(s_{\mathrm{c}}, s_{\mathrm{d}}\right)$ if and only if (2.12) and (2.13) are satisfied as equalities with $\varepsilon=0$ for all $k \in \overline{\mathbb{Z}}_{+}$.

\section{Vector dissipativity theory for large-scale impulsive dynamical systems}

In this section, we extend the notion of dissipative impulsive dynamical systems to develop the generalized notion of vector dissipativity for large-scale impulsive dynamical systems. We begin by considering input/state-dependent impulsive dynamical systems $\mathscr{G}$ of the form

$$
\begin{array}{cl}
\dot{x}(t)=F_{\mathrm{c}}\left(x(t), u_{\mathrm{c}}(t)\right), \quad x\left(t_{0}\right)=x_{0}, \quad\left(x(t), u_{\mathrm{c}}(t)\right) \notin \mathscr{Z}, \quad t \geq t_{0}, \\
\Delta x(t)=F_{\mathrm{d}}\left(x(t), u_{\mathrm{d}}(t)\right), \quad\left(x(t), u_{\mathrm{c}}(t)\right) \in \mathscr{Z}, \\
y_{\mathrm{c}}(t)=H_{\mathrm{c}}\left(x(t), u_{\mathrm{c}}(t)\right), \quad\left(x(t), u_{\mathrm{c}}(t)\right) \notin \mathscr{Z}, \\
y_{\mathrm{d}}(t)=H_{\mathrm{d}}\left(x(t), u_{\mathrm{d}}(t)\right), \quad\left(x(t), u_{\mathrm{c}}(t)\right) \in \mathscr{L},
\end{array}
$$

where $x(t) \in \mathscr{D} \subseteq \mathbb{R}^{n}, t \geq t_{0}, u_{\mathrm{c}} \in \mathcal{U}_{\mathrm{c}} \subseteq \mathbb{R}^{m_{\mathrm{c}}}, u_{\mathrm{d}} \in \boldsymbol{U}_{\mathrm{d}} \subseteq \mathbb{R}^{m_{\mathrm{d}}}, y_{\mathrm{c}} \in \mathscr{Y}_{\mathrm{c}} \subseteq \mathbb{R}^{l_{\mathrm{c}}}, y_{\mathrm{d}} \in \mathscr{Y}_{\mathrm{d}} \subseteq$ $\mathbb{R}^{l_{\mathrm{d}}}, F_{\mathrm{c}}: \mathscr{D} \times \mathcal{U}_{\mathrm{c}} \rightarrow \mathbb{R}^{n}, F_{\mathrm{d}}: \mathscr{D} \times \mathcal{U}_{\mathrm{d}} \rightarrow \mathbb{R}^{n}, H_{\mathrm{c}}: \mathscr{D} \times \mathcal{U}_{\mathrm{c}} \rightarrow \mathscr{Y}_{\mathrm{c}}, H_{\mathrm{d}}: \mathscr{D} \times \mathcal{U}_{\mathrm{d}} \rightarrow \mathscr{Y}_{\mathrm{d}}$, $\mathscr{D}$ is an open set with $0 \in \mathscr{D}, \mathscr{L} \subset \mathscr{D} \times \mathcal{U}_{\mathrm{c}}$, and $F_{\mathrm{c}}(0,0)=0$. Here, we assume that $\mathscr{G}$ represents a large-scale impulsive dynamical system composed of $q$ interconnected controlled impulsive subsystems $\mathscr{G}_{i}$ such that, for all $i=1, \ldots, q$,

$$
\begin{gathered}
F_{\mathrm{c} i}\left(x, u_{\mathrm{c} i}\right)=f_{\mathrm{c} i}\left(x_{i}\right)+\mathscr{I}_{\mathrm{c} i}(x)+G_{\mathrm{c} i}\left(x_{i}\right) u_{\mathrm{c} i}, \\
F_{\mathrm{d} i}\left(x, u_{\mathrm{d} i}\right)=f_{\mathrm{d} i}\left(x_{i}\right)+\mathscr{I}_{\mathrm{d} i}(x)+G_{\mathrm{d} i}\left(x_{i}\right) u_{\mathrm{d} i}, \\
H_{\mathrm{c} i}\left(x_{i}, u_{\mathrm{c} i}\right)=h_{\mathrm{c} i}\left(x_{i}\right)+J_{\mathrm{c} i}\left(x_{i}\right) u_{\mathrm{c} i}, \\
H_{\mathrm{d} i}\left(x_{i}, u_{\mathrm{d} i}\right)=h_{\mathrm{d} i}\left(x_{i}\right)+J_{\mathrm{d} i}\left(x_{i}\right) u_{\mathrm{d} i},
\end{gathered}
$$


where $x_{i} \in \mathscr{D}_{i} \subseteq \mathbb{R}^{n_{i}}, u_{\mathrm{c} i} \in \mathcal{U}_{\mathrm{c} i} \subseteq \mathbb{R}^{m_{\mathrm{c} i}}, u_{\mathrm{d} i} \in \boldsymbol{U}_{\mathrm{d} i} \subseteq \mathbb{R}^{m_{\mathrm{d} i}}, y_{\mathrm{c} i} \triangleq H_{\mathrm{c} i}\left(x_{i}, u_{\mathrm{c} i}\right) \in \mathscr{Y}_{\mathrm{c} i} \subseteq \mathbb{R}^{l_{c i}}$, $y_{\mathrm{d} i} \triangleq H_{\mathrm{d} i}\left(x_{i}, u_{\mathrm{d} i}\right) \in \mathscr{Y}_{\mathrm{d} i} \subseteq \mathbb{R}^{l_{\mathrm{d} i}},\left(\left(u_{\mathrm{c} i}, u_{\mathrm{d} i}\right),\left(y_{\mathrm{c} i}, y_{\mathrm{d} i}\right)\right)$ is the hybrid input-output pair for the $i$ th subsystem, $f_{\mathrm{c} i}: \mathbb{R}^{n_{i}} \rightarrow \mathbb{R}^{n_{i}}$ and $\mathscr{I}_{\mathrm{c} i}: \mathscr{D} \rightarrow \mathbb{R}^{n_{i}}$ are Lipschitz continuous and satisfy $f_{\mathrm{c} i}(0)$ $=0$ and $\Phi_{\mathrm{c} i}(0)=0, f_{\mathrm{d} i}: \mathbb{R}^{n_{i}} \rightarrow \mathbb{R}^{n_{i}}$ and $\mathscr{I}_{\mathrm{d} i}: \mathscr{D} \rightarrow \mathbb{R}^{n_{i}}$ are continuous, $G_{\mathrm{c} i}: \mathbb{R}^{n_{i}} \rightarrow \mathbb{R}^{n_{i} \times m_{\mathrm{c} i}}$ and $G_{\mathrm{d} i}: \mathbb{R}^{n_{i}} \rightarrow \mathbb{R}^{n_{i} \times m_{\mathrm{d} i}}$ are continuous, $h_{\mathrm{c} i}: \mathbb{R}^{n_{i}} \rightarrow \mathbb{R}^{l_{c i}}$ and satisfies $h_{\mathrm{c} i}(0)=0, h_{\mathrm{d} i}: \mathbb{R}^{n_{i}} \rightarrow$ $\mathbb{R}^{l_{\mathrm{d} i}}, J_{\mathrm{c} i}: \mathbb{R}^{n_{i}} \rightarrow \mathbb{R}^{l_{\mathrm{c} i} \times m_{\mathrm{c} i}}, J_{\mathrm{d} i}: \mathbb{R}^{n_{i}} \rightarrow \mathbb{R}^{l_{\mathrm{d} i} \times m_{\mathrm{d} i}}, \sum_{i=1}^{q} n_{i}=n, \sum_{i=1}^{q} m_{\mathrm{c} i}=m_{\mathrm{c}}, \sum_{i=1}^{q} m_{\mathrm{d} i}=m_{\mathrm{d}}$, $\sum_{i=1}^{q} l_{\mathrm{c} i}=l_{\mathrm{c}}$, and $\sum_{i=1}^{q} l_{\mathrm{d} i}=l_{\mathrm{d}}$. Furthermore, for the large-scale impulsive dynamical system $\mathscr{G}$, we assume that the required properties for the existence and uniqueness of solutions are satisfied; that is, for each $i \in\{1, \ldots, q\}, u_{\mathrm{c} i}(\cdot)$ satisfies sufficient regularity conditions such that the system (3.1), (3.2) has a unique solution forward in time. We define the composite input and composite output for the large-scale impulsive dynamical system $\mathscr{G}$ as $u_{\mathrm{c}} \triangleq\left[u_{\mathrm{c} 1}^{\mathrm{T}}, \ldots, u_{\mathrm{c} q}^{\mathrm{T}}\right]^{\mathrm{T}}, u_{\mathrm{d}} \triangleq\left[u_{\mathrm{d} 1}^{\mathrm{T}}, \ldots, u_{\mathrm{d} q}^{\mathrm{T}}\right]^{\mathrm{T}}, y_{\mathrm{c}} \triangleq\left[y_{\mathrm{c} 1}^{\mathrm{T}}, \ldots, y_{\mathrm{c} q}^{\mathrm{T}}\right]^{\mathrm{T}}$, and $y_{\mathrm{d}} \triangleq\left[y_{\mathrm{d} 1}^{\mathrm{T}}, \ldots, y_{\mathrm{d} q}^{\mathrm{T}}\right]^{\mathrm{T}}$, respectively.

Definition 3.1. For the large-scale impulsive dynamical system $\varphi_{\mathcal{G}}$ given by (3.1), (3.2), (3.3), and (3.4), a function $\left(S_{\mathrm{c}}\left(u_{\mathrm{c}}, y_{\mathrm{c}}\right), S_{\mathrm{d}}\left(u_{\mathrm{d}}, y_{\mathrm{d}}\right)\right)$, where $S_{\mathrm{c}}\left(u_{\mathrm{c}}, y_{\mathrm{c}}\right) \triangleq\left[s_{\mathrm{c} 1}\left(u_{\mathrm{c} 1}, y_{\mathrm{c} 1}\right), \ldots\right.$, $\left.s_{\mathrm{c} q}\left(u_{\mathrm{c} q}, y_{\mathrm{c} q}\right)\right]^{\mathrm{T}}, s_{\mathrm{d}}\left(u_{\mathrm{d}}, y_{\mathrm{d}}\right) \triangleq\left[s_{\mathrm{d} 1}\left(u_{\mathrm{d} 1}, y_{\mathrm{d} 1}\right), \ldots, s_{\mathrm{d} q}\left(u_{\mathrm{d} q}, y_{\mathrm{d} q}\right)\right]^{\mathrm{T}}, s_{\mathrm{c} i}: \cup_{\mathrm{c} i} \times \mathscr{Y}_{\mathrm{c} i} \rightarrow \mathbb{R}$, and $s_{\mathrm{d} i}:$ $u_{\mathrm{d} i} \times \mathscr{Y}_{\mathrm{d} i} \rightarrow \mathbb{R}, i=1, \ldots, q$, such that $S_{\mathrm{c}}(0,0)=0$ and $S_{\mathrm{d}}(0,0)=0$, is called a vector $h y$ brid supply rate if it is locally componentwise integrable for all input-output pairs satisfying (3.1), (3.2), (3.3), and (3.4); that is, for every $i \in\{1, \ldots, q\}$ and for all inputoutput pairs $u_{\mathrm{c} i} \in \mathcal{U}_{\mathrm{c} i}, y_{\mathrm{c} i} \in \mathscr{Y}_{\mathrm{c} i}$ satisfying (3.1), (3.2), (3.3), and (3.4), $s_{\mathrm{c} i}(\cdot, \cdot)$ satisfies $\int_{t}^{\hat{t}}\left|s_{c i}\left(u_{c i}(s), y_{c i}(s)\right)\right| \mathrm{d} s<\infty, t, \hat{t} \geq t_{0}$.

Note that since all input-output pairs $u_{\mathrm{d} i}\left(t_{k}\right) \in \mathcal{U}_{\mathrm{d} i}, y_{\mathrm{d} i}\left(t_{k}\right) \in \mathscr{Y}_{\mathrm{d} i}$ are defined for dis-

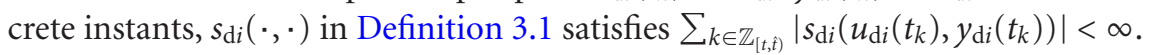

Definition 3.2. The large-scale impulsive dynamical system $\mathscr{G}$ given by (3.1), (3.2), (3.3), and (3.4) is vector dissipative (resp., exponentially vector dissipative) with respect to the vector hybrid supply rate $\left(S_{\mathrm{c}}, S_{\mathrm{d}}\right)$ if there exist a continuous, nonnegative-definite vector function $V_{\mathrm{s}}=\left[v_{\mathrm{s} 1}, \ldots, v_{\mathrm{s} q}\right]^{\mathrm{T}}: \mathscr{D} \rightarrow \overline{\mathbb{R}}_{+}^{q}$, called a vector storage function, and an essentially nonnegative dissipation matrix $W \in \mathbb{R}^{q \times q}$ such that $V_{\mathrm{s}}(0)=0, W$ is semistable (resp., asymptotically stable), and the vector hybrid dissipation inequality

$$
\begin{aligned}
V_{\mathrm{s}}(x(T)) \leq \leq & e^{W\left(T-t_{0}\right)} V_{\mathrm{s}}\left(x\left(t_{0}\right)\right)+\int_{t_{0}}^{T} e^{W(T-t)} S_{\mathrm{c}}\left(u_{\mathrm{c}}(t), y_{\mathrm{c}}(t)\right) \mathrm{d} t \\
& +\sum_{k \in \mathbb{Z}_{\left[t_{0}, T\right)}} e^{W\left(T-t_{k}\right)} S_{\mathrm{d}}\left(u_{\mathrm{d}}\left(t_{k}\right), y_{\mathrm{d}}\left(t_{k}\right)\right), \quad T \geq t_{0},
\end{aligned}
$$

is satisfied, where $x(t), t \geq t_{0}$, is the solution to (3.1), (3.2), (3.3), and (3.4) with $\left(u_{\mathrm{c}}(t)\right.$, $\left.u_{\mathrm{d}}\left(t_{k}\right)\right) \in u_{\mathrm{c}} \times u_{\mathrm{d}}$ and $x\left(t_{0}\right)=x_{0}$. The large-scale impulsive dynamical system $\mathscr{G}$ given by (3.1), (3.2), (3.3), and (3.4) is vector lossless with respect to the vector hybrid supply rate $\left(S_{\mathrm{c}}, S_{\mathrm{d}}\right)$ if the vector hybrid dissipation inequality is satisfied as an equality with $W$ semistable.

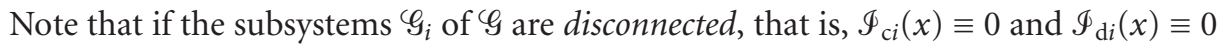
for all $i=1, \ldots, q$, and $-W \in \mathbb{R}^{q \times q}$ is diagonal and nonnegative-definite, then it follows 
234 Vector dissipativity and large-scale impulsive systems

from Definition 3.2 that each of disconnected subsystems $\mathscr{G}_{i}$ is dissipative or exponentially dissipative in the sense of Definition 2.8. A similar remark holds in the case where $q=1$. Next, define the vector available storage of the large-scale impulsive dynamical system $\mathscr{G}$ by

$$
\begin{aligned}
V_{\mathrm{a}}\left(x_{0}\right) \triangleq- & \inf _{T \geq t_{0},\left(u_{\mathrm{c}}(\cdot), u_{\mathrm{d}}(\cdot)\right)}\left[\int_{t_{0}}^{T} e^{-W\left(t-t_{0}\right)} S_{\mathrm{c}}\left(u_{\mathrm{c}}(t), y_{\mathrm{c}}(t)\right) \mathrm{d} t\right. \\
& \left.+\sum_{k \in \mathbb{Z}_{\left[t_{0}, T\right)}} e^{-W\left(t_{k}-t_{0}\right)} S_{\mathrm{d}}\left(u_{\mathrm{d}}\left(t_{k}\right), y_{\mathrm{d}}\left(t_{k}\right)\right)\right],
\end{aligned}
$$

where $x(t), t \geq t_{0}$, is the solution to (3.1), (3.2), (3.3), and (3.4) with $x\left(t_{0}\right)=x_{0}$ and admissible inputs $\left(u_{\mathrm{c}}, u_{\mathrm{d}}\right) \in \boldsymbol{U}_{\mathrm{c}} \times \boldsymbol{U}_{\mathrm{d}}$. The infimum in (3.7) is taken componentwise which implies that for different elements of $V_{\mathrm{a}}(\cdot)$, the infimum is calculated separately. Note that $V_{\mathrm{a}}\left(x_{0}\right) \geq \geq 0, x_{0} \in \mathscr{D}$, since $V_{\mathrm{a}}\left(x_{0}\right)$ is the infimum over a set of vectors containing the zero vector $\left(T=t_{0}\right)$.

Theorem 3.3. Consider the large-scale impulsive dynamical system $G$ given by (3.1), (3.2), (3.3), and (3.4) and assume that $\mathscr{G}$ is completely reachable. Then $\mathscr{G}$ is vector dissipative (resp., exponentially vector dissipative) with respect to the vector hybrid supply rate $\left(S_{\mathrm{c}}, S_{\mathrm{d}}\right)$ if and only if there exist a continuous, nonnegative-definite vector function $V_{s}: \mathscr{D} \rightarrow \overline{\mathbb{R}}_{+}^{q}$ and an essentially nonnegative dissipation matrix $W \in \mathbb{R}^{q \times q}$ such that $V_{\mathrm{s}}(0)=0, W$ is semistable (resp., asymptotically stable), and for all $k \in \overline{\mathbb{Z}}_{+}$,

$$
\begin{gathered}
V_{\mathrm{s}}(x(\hat{t})) \leq \leq e^{W(\hat{t}-t)} V_{\mathrm{s}}(x(t))+\int_{t}^{\hat{t}} e^{W(\hat{t}-s)} S_{\mathrm{c}}\left(u_{\mathrm{c}}(s), y_{\mathrm{c}}(s)\right) \mathrm{d} s, \quad t_{k}<t \leq \hat{t} \leq t_{k+1}, \\
V_{\mathrm{s}}\left(x\left(t_{k}\right)+F_{\mathrm{d}}\left(x\left(t_{k}\right), u_{\mathrm{d}}\left(t_{k}\right)\right)\right) \leq \leq V_{\mathrm{s}}\left(x\left(t_{k}\right)\right)+S_{\mathrm{d}}\left(u_{\mathrm{d}}\left(t_{k}\right), y_{\mathrm{d}}\left(t_{k}\right)\right) .
\end{gathered}
$$

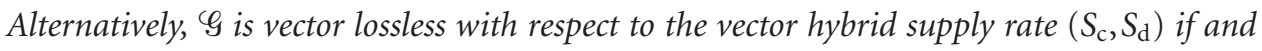
only if (3.8) and (3.9) are satisfied as equalities with $W$ semistable.

Proof. Let $k \in \overline{\mathbb{Z}}_{+}$and suppose $\mathscr{G}$ is vector dissipative (resp., exponentially vector dissipative) with respect to the vector hybrid supply rate $\left(S_{c}, S_{d}\right)$. Then, there exist a continuous nonnegative-definite vector function $V_{\mathrm{s}}: \mathscr{D} \rightarrow \overline{\mathbb{R}}_{+}^{q}$ and an essentially nonnegative matrix $W \in \mathbb{R}^{q \times q}$ such that (3.6) holds. Now, since for $t_{k}<t \leq \hat{t} \leq t_{k+1}, \mathbb{Z}_{[t, \hat{t})}=\varnothing$, (3.8) is immediate. Next, it follows from (3.6) that

$$
\begin{aligned}
V_{\mathrm{s}}\left(x\left(t_{k}^{+}\right)\right) \leq \leq & e^{W\left(t_{k}^{+}-t_{k}\right)} V_{\mathrm{s}}\left(x\left(t_{k}\right)\right)+\int_{t_{k}}^{t_{k}^{+}} e^{W\left(t_{k}^{+}-s\right)} S_{\mathrm{c}}\left(u_{\mathrm{c}}(s), y_{\mathrm{c}}(s)\right) \mathrm{d} s \\
& +\sum_{k \in \mathbb{Z}_{\left[t_{k}, t_{k}^{+}\right)}} e^{W\left(t_{k}^{+}-t_{k}\right)} S_{\mathrm{d}}\left(u_{\mathrm{d}}\left(t_{k}\right), y_{\mathrm{d}}\left(t_{k}\right)\right),
\end{aligned}
$$

which, since $\mathbb{Z}_{\left[t_{k}, t_{k}^{+}\right)}=k$, implies (3.9).

Conversely, suppose (3.8) and (3.9) hold and let $\hat{t} \geq t \geq t_{0}$ and $\mathbb{Z}_{[t, \hat{t})}=\{i, i+1, \ldots, j\}$. (Note that if $\mathbb{Z}_{[t, \hat{t})}=\varnothing$, the converse result is a direct consequence of (3.8).) If $\mathbb{Z}_{[t, \hat{t})} \neq \varnothing$, 
it follows from (3.8) and (3.9) that

$$
\begin{aligned}
& V_{\mathrm{s}}(x(\hat{t}))-e^{W(\hat{t}-t)} V_{\mathrm{s}}(x(t)) \\
& =V_{\mathrm{s}}(x(\hat{t}))-e^{W\left(\hat{t}-t_{j}^{+}\right)} V_{\mathrm{s}}\left(x\left(t_{j}^{+}\right)\right) \\
& +e^{W\left(\hat{t}-t_{j}^{+}\right)} V_{\mathrm{s}}\left(x\left(t_{j}^{+}\right)\right)-e^{W\left(\hat{t}-t_{j-1}^{+}\right)} V_{\mathrm{s}}\left(x\left(t_{j-1}^{+}\right)\right) \\
& +e^{W\left(\hat{t}-t_{j-1}^{+}\right)} V_{\mathrm{s}}\left(x\left(t_{j-1}^{+}\right)\right)-\cdots-e^{W\left(\hat{t}-t_{i}^{+}\right)} V_{\mathrm{s}}\left(x\left(t_{i}^{+}\right)\right) \\
& +e^{W\left(\hat{t}-t_{i}^{+}\right)} V_{s}\left(x\left(t_{i}^{+}\right)\right)-e^{W(\hat{t}-t)} V_{s}(x(t)) \\
& =V_{\mathrm{s}}(x(\hat{t}))-e^{W\left(\hat{t}-t_{j}\right)} V_{\mathrm{s}}\left(x\left(t_{j}^{+}\right)\right) \\
& +e^{W\left(\hat{t}-t_{j}\right)} V_{\mathrm{s}}\left(x\left(t_{j}\right)+F_{\mathrm{d}}\left(x\left(t_{j}\right), u_{\mathrm{d}}\left(t_{j}\right)\right)\right)-e^{W\left(\hat{t}-t_{j}\right)} V_{\mathrm{s}}\left(x\left(t_{j}\right)\right) \\
& +e^{W\left(\hat{t}-t_{j}\right)} V_{s}\left(x\left(t_{j}\right)\right)-e^{W\left(\hat{t}-t_{j-1}^{+}\right)} V_{s}\left(x\left(t_{j-1}^{+}\right)\right) \\
& +\cdots+e^{W\left(\hat{t}-t_{i}\right)} V_{\mathrm{s}}\left(x\left(t_{i}\right)+F_{\mathrm{d}}\left(x\left(t_{i}\right), u_{\mathrm{d}}\left(t_{i}\right)\right)\right)-e^{W\left(\hat{t}-t_{i}\right)} V_{\mathrm{s}}\left(x\left(t_{i}\right)\right) \\
& +e^{W\left(\hat{t}-t_{i}\right)} V_{s}\left(x\left(t_{i}\right)\right)-e^{W(\hat{t}-t)} V_{\mathrm{s}}(x(t)) \\
& =V_{\mathrm{s}}(x(\hat{t}))-e^{W\left(\hat{t}-t_{j}\right)} V_{\mathrm{s}}\left(x\left(t_{j}^{+}\right)\right) \\
& +e^{W\left(\hat{t}-t_{j}\right)}\left[V_{\mathrm{s}}\left(x\left(t_{j}\right)+F_{\mathrm{d}}\left(x\left(t_{j}\right), u_{\mathrm{d}}\left(t_{j}\right)\right)\right)-V_{\mathrm{s}}\left(x\left(t_{j}\right)\right)\right] \\
& +e^{W\left(\hat{t}-t_{j}\right)}\left[V_{s}\left(x\left(t_{j}\right)\right)-e^{W\left(t_{j}-t_{j-1}\right)} V_{s}\left(x\left(t_{j-1}^{+}\right)\right)\right] \\
& +\cdots+e^{W\left(\hat{t}-t_{i}\right)}\left[V_{\mathrm{s}}\left(x\left(t_{i}\right)+F_{\mathrm{d}}\left(x\left(t_{i}\right), u_{\mathrm{d}}\left(t_{i}\right)\right)\right)-V_{\mathrm{s}}\left(x\left(t_{i}\right)\right)\right] \\
& +e^{W\left(\hat{t}-t_{i}\right)}\left[V_{\mathrm{s}}\left(x\left(t_{i}\right)\right)-e^{W\left(t_{i}-t\right)} V_{\mathrm{s}}(x(t))\right] \\
& \leq \leq \int_{t_{j}}^{\hat{t}} e^{W(\hat{t}-s)} S_{\mathrm{c}}\left(u_{\mathrm{c}}(s), y_{\mathrm{c}}(s)\right) \mathrm{d} s+e^{W\left(\hat{t}-t_{j}\right)} S_{\mathrm{d}}\left(u_{\mathrm{d}}\left(t_{j}\right), y_{\mathrm{d}}\left(t_{j}\right)\right) \\
& +e^{W\left(\hat{t}-t_{j}\right)} \int_{t_{j-1}}^{t_{j}} e^{W\left(t_{j}-s\right)} S_{\mathrm{c}}\left(u_{\mathrm{c}}(s), y_{\mathrm{c}}(s)\right) \mathrm{d} s \\
& +\cdots+e^{W\left(\hat{t}-t_{i}\right)} S_{\mathrm{d}}\left(u_{\mathrm{d}}\left(t_{i}\right), y_{\mathrm{d}}\left(t_{i}\right)\right) \\
& +e^{W\left(\hat{t}-t_{i}\right)} \int_{t}^{t_{i}} e^{W\left(t_{i}-s\right)} S_{\mathrm{c}}\left(u_{\mathrm{c}}(s), y_{\mathrm{c}}(s)\right) \mathrm{d} s \\
& =\int_{t}^{\hat{t}} e^{W(\hat{t}-s)} S_{\mathrm{c}}\left(u_{\mathrm{c}}(s), y_{\mathrm{c}}(s)\right) \mathrm{d} s+\sum_{k \in \mathbb{Z}_{[t, \hat{t})}} e^{W\left(\hat{t}-t_{k}\right)} S_{\mathrm{d}}\left(u_{\mathrm{d}}\left(t_{k}\right), y_{\mathrm{d}}\left(t_{k}\right)\right) \text {, }
\end{aligned}
$$

which implies that $\mathscr{G}$ is vector dissipative (resp., exponentially vector dissipative) with respect to the vector hybrid supply rate $\left(S_{\mathrm{c}}, S_{\mathrm{d}}\right)$. Finally, similar constructions show that $G_{\mathrm{S}}$ is vector lossless with respect to the vector hybrid supply rate $\left(S_{\mathrm{c}}, S_{\mathrm{d}}\right)$ if and only if (3.8) and (3.9) are satisfied as equalities with $W$ semistable. 
236 Vector dissipativity and large-scale impulsive systems

Theorem 3.4. Consider the large-scale impulsive dynamical system $G$ given by (3.1), (3.2), (3.3), and (3.4) and assume that $G$ is completely reachable. Let $W \in \mathbb{R}^{q \times q}$ be essentially nonnegative and semistable (resp., asymptotically stable). Then

$$
\begin{aligned}
& \int_{t_{0}}^{T} e^{-W\left(t-t_{0}\right)} S_{\mathrm{c}}\left(u_{\mathrm{c}}(t), y_{\mathrm{c}}(t)\right) \mathrm{d} t \\
& \quad+\sum_{k \in \mathbb{Z}_{\left[t_{0}, T\right)}} e^{-W\left(t_{k}-t_{0}\right)} S_{\mathrm{d}}\left(u_{\mathrm{d}}\left(t_{k}\right), y_{\mathrm{d}}\left(t_{k}\right)\right) \geq \geq 0, \quad T \geq t_{0},
\end{aligned}
$$

for $x\left(t_{0}\right)=0$ and $\left(u_{\mathrm{c}}, u_{\mathrm{d}}\right) \in u_{\mathrm{c}} \times u_{\mathrm{d}}$ if and only if $V_{\mathrm{a}}(0)=0$ and $V_{\mathrm{a}}(x)$ is finite for all $x \in \mathscr{D}$. Moreover, if (3.12) holds, then $V_{\mathrm{a}}(x), x \in \mathscr{D}$, is a vector storage function for $\mathscr{G}$ and hence $G$ is vector dissipative (resp., exponentially vector dissipative) with respect to the vector hybrid supply rate $\left(S_{\mathrm{c}}\left(u_{\mathrm{c}}, y_{\mathrm{c}}\right), S_{\mathrm{d}}\left(u_{\mathrm{d}}, y_{\mathrm{d}}\right)\right)$.

Proof. Suppose $V_{\mathrm{a}}(0)=0$ and $V_{\mathrm{a}}(x), x \in \mathscr{D}$, is finite. Then

$$
\begin{aligned}
0=V_{\mathrm{a}}(0)=-\inf _{T \geq t_{0},\left(u_{\mathrm{c}}(\cdot), u_{\mathrm{d}}(\cdot)\right)}[ & \int_{t_{0}}^{T} e^{-W\left(t-t_{0}\right)} S_{\mathrm{c}}\left(u_{\mathrm{c}}(t), y_{\mathrm{c}}(t)\right) \mathrm{d} t \\
& \left.+\sum_{k \in \mathbb{Z}_{\left[t_{0}, T\right)}} e^{-W\left(t_{k}-t_{0}\right)} S_{\mathrm{d}}\left(u_{\mathrm{d}}\left(t_{k}\right), y_{\mathrm{d}}\left(t_{k}\right)\right)\right],
\end{aligned}
$$

which implies (3.12).

Next, suppose (3.12) holds. Then for $x\left(t_{0}\right)=0$,

$$
\begin{aligned}
-\inf _{T \geq t_{0},\left(u_{\mathrm{c}}(\cdot), u_{\mathrm{d}}(\cdot)\right)} & {\left[\int_{t_{0}}^{T} e^{-W\left(t-t_{0}\right)} S_{\mathrm{c}}\left(u_{\mathrm{c}}(t), y_{\mathrm{c}}(t)\right) \mathrm{d} t\right.} \\
& \left.+\sum_{k \in \mathbb{Z}_{\left[t_{0}, T\right)}} e^{-W\left(t_{k}-t_{0}\right)} S_{\mathrm{d}}\left(u_{\mathrm{d}}\left(t_{k}\right), y_{\mathrm{d}}\left(t_{k}\right)\right)\right] \leq \leq 0,
\end{aligned}
$$

which implies that $V_{\mathrm{a}}(0) \leq \leq 0$. However, since $V_{\mathrm{a}}\left(x_{0}\right) \geq \geq 0, x_{0} \in \mathscr{D}$, it follows that $V_{\mathrm{a}}(0)$ $=0$. Moreover, since $\mathscr{G}$ is completely reachable, it follows that for every $x_{0} \in \mathscr{D}$, there exist $\hat{t}>t_{0}$ and an admissible input $u(\cdot)$ defined on $\left[t_{0}, \hat{t}\right]$ such that $x(\hat{t})=x_{0}$. Now, since (3.12) holds for $x\left(t_{0}\right)=0$, it follows that for all admissible $\left(u_{\mathrm{c}}, y_{\mathrm{c}}\right) \in \boldsymbol{U}_{\mathrm{c}} \times \mathscr{Y}_{\mathrm{c}}$ and $\left(u_{\mathrm{d}}, y_{\mathrm{d}}\right) \in$ $u_{\mathrm{d}} \times y_{\mathrm{d}}$

$$
\begin{aligned}
& \int_{t_{0}}^{T} e^{-W\left(t-t_{0}\right)} S_{\mathrm{c}}\left(u_{\mathrm{c}}(t), y_{\mathrm{c}}(t)\right) \mathrm{d} t \\
& \quad+\sum_{k \in \mathbb{Z}_{\left[t_{0}, T\right)}} e^{-W\left(t_{k}-t_{0}\right)} S_{\mathrm{d}}\left(u_{\mathrm{d}}\left(t_{k}\right), y_{\mathrm{d}}\left(t_{k}\right)\right) \geq \geq 0, \quad T \geq \hat{t}
\end{aligned}
$$


or, equivalently, multiplying (3.15) by the nonnegative matrix $e^{W\left(\hat{t}-t_{0}\right)}, \hat{t} \geq t_{0}$, yields

$$
\begin{aligned}
& -\int_{\hat{t}}^{T} e^{-(t-\hat{t})} S_{\mathrm{c}}\left(u_{\mathrm{c}}(t), y_{\mathrm{c}}(t)\right) \mathrm{d} t-\sum_{k \in \mathbb{Z}_{[\hat{t}, T)}} e^{-W\left(t_{k}-\hat{t}\right)} S_{\mathrm{d}}\left(u_{\mathrm{d}}\left(t_{k}\right), u_{\mathrm{d}}\left(t_{k}\right)\right) \\
& \quad \leq \leq \int_{t_{0}}^{\hat{t}} e^{-W(t-\hat{t})} S_{\mathrm{c}}\left(u_{\mathrm{c}}(t), y_{\mathrm{c}}(t)\right) \mathrm{d} t+\sum_{k \in \mathbb{Z}_{\left[t_{0}, \hat{t}\right)}} e^{-W\left(t_{k}-\hat{t}\right)} S_{\mathrm{d}}\left(u_{\mathrm{d}}\left(t_{k}\right), u_{\mathrm{d}}\left(t_{k}\right)\right) \\
& \quad \leq \leq Q\left(x_{0}\right) \ll \infty, \quad T \geq \hat{t},\left(u_{\mathrm{c}}, u_{\mathrm{d}}\right) \in \mathcal{U}_{\mathrm{c}} \times \boldsymbol{U}_{\mathrm{d}},
\end{aligned}
$$

where $Q: \mathscr{D} \rightarrow \mathbb{R}^{q}$. Hence,

$$
\begin{aligned}
V_{\mathrm{a}}\left(x_{0}\right)=-\inf _{T \geq \hat{t},\left(u_{\mathrm{c}}(\cdot), u_{\mathrm{d}}(\cdot)\right)} & {\left[\int_{\hat{t}}^{T} e^{-W(t-\hat{t})} S_{\mathrm{c}}\left(u_{\mathrm{c}}(t), y_{\mathrm{c}}(t)\right) \mathrm{d} t\right.} \\
& \left.+\sum_{k \in \mathbb{Z}_{[\hat{t}, T)}} e^{-W\left(t_{k}-\hat{t}\right)} S_{\mathrm{d}}\left(u_{\mathrm{d}}\left(t_{k}\right), u_{\mathrm{d}}\left(t_{k}\right)\right)\right] \\
\leq \leq Q\left(x_{0}\right) \ll \infty, \quad & x_{0} \in \mathscr{D},
\end{aligned}
$$

which implies that $V_{\mathrm{a}}\left(x_{0}\right), x_{0} \in \mathscr{D}$, is finite.

Finally, since (3.12) implies that $V_{\mathrm{a}}(0)=0$ and $V_{\mathrm{a}}(x), x \in \mathscr{D}$, is finite, it follows from the definition of the vector available storage that

$$
\begin{aligned}
-V_{\mathrm{a}}\left(x_{0}\right) \leq \leq & \int_{t_{0}}^{T} e^{-W\left(t-t_{0}\right)} S_{\mathrm{c}}\left(u_{\mathrm{c}}(t), y_{\mathrm{c}}(t)\right) \mathrm{d} t \\
& +\sum_{k \in \mathbb{Z}_{\left[t_{0}, T\right)}} e^{-W\left(t_{k}-t_{0}\right)} S_{\mathrm{d}}\left(u_{\mathrm{d}}\left(t_{k}\right), u_{\mathrm{d}}\left(t_{k}\right)\right) \\
= & \int_{t_{0}}^{t_{\mathrm{f}}} e^{-W\left(t-t_{0}\right)} S_{\mathrm{c}}\left(u_{\mathrm{c}}(t), y_{\mathrm{c}}(t)\right) \mathrm{d} t \\
& +\sum_{k \in \mathbb{Z}_{\left[t_{0}, t_{\mathrm{f}}\right)}} e^{-W\left(t_{k}-t_{0}\right)} S_{\mathrm{d}}\left(u_{\mathrm{d}}\left(t_{k}\right), u_{\mathrm{d}}\left(t_{k}\right)\right) \\
& +\int_{t_{\mathrm{f}}}^{T} e^{-W\left(t-t_{0}\right)} S_{\mathrm{c}}\left(u_{\mathrm{c}}(t), y_{\mathrm{c}}(t)\right) \mathrm{d} t \\
& +\sum_{k \in \mathbb{Z}_{\left[t_{\mathrm{f}}, T\right)}} e^{-W\left(t_{k}-t_{0}\right)} S_{\mathrm{d}}\left(u_{\mathrm{d}}\left(t_{k}\right), u_{\mathrm{d}}\left(t_{k}\right)\right), \quad T \geq t_{0} .
\end{aligned}
$$


Now, multiplying (3.18) by the nonnegative matrix $e^{W\left(t_{\mathrm{f}}-t_{0}\right)}, t_{\mathrm{f}} \geq t_{0}$, it follows that

$$
\begin{gathered}
e^{W\left(t_{\mathrm{f}}-t_{0}\right)} V_{\mathrm{a}}\left(x_{0}\right)+\int_{t_{0}}^{t_{\mathrm{f}}} e^{W\left(t_{\mathrm{f}}-t\right)} S_{\mathrm{c}}\left(u_{\mathrm{c}}(t), y_{\mathrm{c}}(t)\right) \mathrm{d} t+\sum_{k \in \mathbb{Z}_{\left[t_{0}, t_{\mathrm{f}}\right)}} e^{W\left(t_{\mathrm{f}}-t_{k}\right)} S_{\mathrm{d}}\left(u_{\mathrm{d}}\left(t_{k}\right), u_{\mathrm{d}}\left(t_{k}\right)\right) \\
\geq \geq-\inf _{T \geq t_{\mathrm{f}},\left(u_{\mathrm{c}}(\cdot), u_{\mathrm{d}}(\cdot)\right)}\left[\int_{t_{\mathrm{f}}}^{T} e^{-W\left(t-t_{\mathrm{f}}\right)} S_{\mathrm{c}}\left(u_{\mathrm{c}}(t), y_{\mathrm{d}}(t)\right) \mathrm{d} t\right. \\
\left.+\sum_{k \in \mathbb{Z}_{\left[t_{\mathrm{f}}, T\right)}} e^{-W\left(t_{k}-t_{\mathrm{f}}\right)} S_{\mathrm{d}}\left(u_{\mathrm{d}}\left(t_{k}\right), u_{\mathrm{d}}\left(t_{k}\right)\right)\right] \\
=V_{\mathrm{a}}\left(x\left(t_{\mathrm{f}}\right)\right),
\end{gathered}
$$

which implies that $V_{\mathrm{a}}(x), x \in \mathscr{D}$, is a vector storage function and hence $\mathscr{G}$ is vector dissipative (resp., exponentially vector dissipative) with respect to the vector hybrid supply rate $\left(S_{\mathrm{c}}\left(u_{\mathrm{c}}, y_{\mathrm{c}}\right), S_{\mathrm{d}}\left(u_{\mathrm{d}}, y_{\mathrm{d}}\right)\right)$.

It follows from Lemma 2.6 that if $W \in \mathbb{R}^{q \times q}$ is essentially nonnegative and semistable (resp., asymptotically stable), then there exist a scalar $\alpha \geq 0$ (resp., $\alpha>0$ ) and a nonnegative vector $p \in \overline{\mathbb{R}}_{+}^{q}, p \neq 0$, (resp., $p \in \mathbb{R}_{+}^{q}$ ) such that (2.2) holds. In this case,

$$
\begin{aligned}
p^{\mathrm{T}} e^{W t} & =p^{\mathrm{T}}\left[I_{q}+W t+\frac{1}{2} W^{2} t^{2}+\cdots\right] \\
& =p^{\mathrm{T}}\left[I_{q}-\alpha t I_{q}+\frac{1}{2} \alpha^{2} t^{2} I_{q}+\cdots\right]=e^{-\alpha t} p^{\mathrm{T}}, \quad t \in \mathbb{R} .
\end{aligned}
$$

Using (3.20), we define the (scalar) available storage for the large-scale impulsive dynamical system $\mathscr{G}$ by

$$
\begin{aligned}
v_{\mathrm{a}}\left(x_{0}\right) \triangleq-\inf _{T \geq t_{0},\left(u_{\mathrm{c}}(\cdot), u_{\mathrm{d}}(\cdot)\right)} & {\left[\int_{t_{0}}^{T} p^{\mathrm{T}} e^{-W\left(t-t_{0}\right)} S_{\mathrm{c}}\left(u_{\mathrm{c}}(t), y_{\mathrm{c}}(t)\right) \mathrm{d} t\right.} \\
& \left.+\sum_{k \in \mathbb{Z}_{\left[t_{0}, T\right)}} p^{\mathrm{T}} e^{-W\left(t_{k}-t_{0}\right)} S_{\mathrm{d}}\left(u_{\mathrm{d}}\left(t_{k}\right), y_{\mathrm{d}}\left(t_{k}\right)\right)\right] \\
=-\inf _{T \geq t_{0},\left(u_{\mathrm{c}}(\cdot), u_{\mathrm{d}}(\cdot)\right)} & {\left[\int_{t_{0}}^{T} e^{\alpha\left(t-t_{0}\right)} s_{\mathrm{c}}\left(u_{\mathrm{c}}(t), y_{\mathrm{c}}(t)\right) \mathrm{d} t\right.} \\
& \left.+\sum_{k \in \mathbb{Z}_{\left[t_{0}, T\right)}} e^{\alpha\left(t_{k}-t_{0}\right)} s_{\mathrm{d}}\left(u_{\mathrm{d}}\left(t_{k}\right), y_{\mathrm{d}}\left(t_{k}\right)\right)\right],
\end{aligned}
$$

where $s_{\mathrm{c}}: \boldsymbol{U}_{\mathrm{c}} \times \mathscr{y}_{\mathrm{c}} \rightarrow \mathbb{R}$ and $s_{\mathrm{d}}: \boldsymbol{U}_{\mathrm{d}} \times \mathscr{Y}_{\mathrm{d}} \rightarrow \mathbb{R}$ defined as $s_{\mathrm{c}}\left(u_{\mathrm{c}}, y_{\mathrm{c}}\right) \triangleq p^{\mathrm{T}} S_{\mathrm{c}}\left(u_{\mathrm{c}}, y_{\mathrm{c}}\right)$ and $s_{\mathrm{d}}\left(u_{\mathrm{d}}, y_{\mathrm{d}}\right) \triangleq p^{\mathrm{T}} S_{\mathrm{d}}\left(u_{\mathrm{d}}, y_{\mathrm{d}}\right)$ form the (scalar) hybrid supply rate $\left(s_{\mathrm{c}}, s_{\mathrm{d}}\right)$ for the large-scale impulsive dynamical system $\mathscr{G}$. Clearly, $v_{\mathrm{a}}(x) \geq 0$ for all $x \in \mathscr{D}$. As in standard hybrid dissipativity theory [10], the available storage $v_{\mathrm{a}}(x), x \in \mathscr{D}$, denotes the maximum amount of (scaled) energy that can be extracted from the large-scale impulsive dynamical system $\mathscr{G}$ at any time $T$. 
The following theorem relates vector storage functions and vector hybrid supply rates to scalar storage functions and scalar hybrid supply rates of large-scale impulsive dynamical systems.

Theorem 3.5. Consider the large-scale impulsive dynamical system Given by (3.1), (3.2), (3.3), and (3.4). Suppose $G$ is vector dissipative (resp., exponentially vector dissipative) with respect to the vector hybrid supply rate $\left(S_{\mathrm{c}}\left(u_{\mathrm{c}}, y_{\mathrm{c}}\right), S_{\mathrm{d}}\left(u_{\mathrm{d}}, y_{\mathrm{d}}\right)\right):\left(\mathcal{u}_{\mathrm{c}} \times \mathscr{Y}_{\mathrm{c}}, \boldsymbol{u}_{\mathrm{d}} \times \mathscr{Y}_{\mathrm{d}}\right) \rightarrow \mathbb{R}^{q} \times$ $\mathbb{R}^{q}$ and with vector storage function $V_{s}: \mathscr{D} \rightarrow \overline{\mathbb{R}}_{+}^{q}$. Then there exists $p \in \overline{\mathbb{R}}_{+}^{q}, p \neq 0$, (resp.,

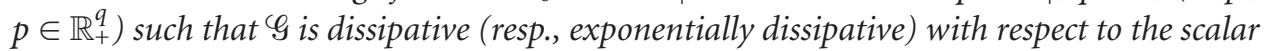
hybrid supply rate $\left(s_{\mathrm{c}}\left(u_{\mathrm{c}}, y_{\mathrm{c}}\right), s_{\mathrm{d}}\left(u_{\mathrm{d}}, y_{\mathrm{d}}\right)\right)=\left(p^{\mathrm{T}} S_{\mathrm{c}}\left(u_{\mathrm{c}}, y_{\mathrm{c}}\right), p^{\mathrm{T}} S_{\mathrm{d}}\left(u_{\mathrm{d}}, y_{\mathrm{d}}\right)\right)$ and with storage function $v_{\mathrm{s}}(x)=p^{\mathrm{T}} V_{\mathrm{s}}(x), x \in \mathscr{D}$. Moreover, in this case, $v_{\mathrm{a}}(x), x \in \mathscr{D}$, is a storage function for $\mathscr{G}$ and

$$
0 \leq v_{\mathrm{a}}(x) \leq v_{\mathrm{s}}(x), \quad x \in \mathscr{D} .
$$

Proof. Suppose $G$ is vector dissipative (resp., exponentially vector dissipative) with respect to the vector hybrid supply rate $\left(S_{\mathrm{c}}\left(u_{\mathrm{c}}, y_{\mathrm{c}}\right), S_{\mathrm{d}}\left(u_{\mathrm{d}}, y_{\mathrm{d}}\right)\right)$. Then there exist an essentially nonnegative, semistable (resp., asymptotically stable) dissipation matrix $W$ and a vector storage function $V_{s}: \mathscr{D} \rightarrow \overline{\mathbb{R}}_{+}^{q}$ such that the dissipation inequality (3.6) holds. Furthermore, it follows from Lemma 2.6 that there exist $\alpha \geq 0$ (resp., $\alpha>0$ ) and a nonzero vector $p \in \overline{\mathbb{R}}_{+}^{q}$ (resp., $p \in \mathbb{R}_{+}^{q}$ ) satisfying (2.2). Hence, premultiplying (3.6) by $p^{\mathrm{T}}$ and using (3.20), it follows that

$$
\begin{aligned}
e^{\alpha T} v_{\mathrm{s}}(x(T)) \leq & e^{\alpha t_{0}} v_{\mathrm{s}}\left(x\left(t_{0}\right)\right)+\int_{t_{0}}^{T} e^{\alpha t} s_{\mathrm{c}}\left(u_{\mathrm{c}}(t), y_{\mathrm{c}}(t)\right) \mathrm{d} t \\
& +\sum_{k \in \mathbb{Z}_{\left[t_{0}, T\right)}} e^{\alpha t_{k}} s_{\mathrm{d}}\left(u_{\mathrm{d}}\left(t_{k}\right), y_{\mathrm{d}}\left(t_{k}\right)\right), \quad T \geq t_{0}, \quad\left(u_{\mathrm{c}}, u_{\mathrm{d}}\right) \in \boldsymbol{u}_{\mathrm{c}} \times \boldsymbol{u}_{\mathrm{d}}
\end{aligned}
$$

where $v_{\mathrm{s}}(x)=p^{\mathrm{T}} V_{\mathrm{s}}(x), x \in \mathscr{D}$, which implies dissipativity (resp., exponential dissipativity) of $\mathscr{G}_{\text {with }}$ respect to the scalar hybrid supply rate $\left(s_{\mathrm{c}}\left(u_{\mathrm{c}}, y_{\mathrm{c}}\right), s_{\mathrm{d}}\left(u_{\mathrm{d}}, y_{\mathrm{d}}\right)\right)$ and with storage function $v_{\mathrm{s}}(x), x \in \mathscr{D}$. Moreover, since $v_{\mathrm{s}}(0)=0$, it follows from (3.23) that for $x\left(t_{0}\right)=0$,

$$
\begin{aligned}
& \int_{t_{0}}^{T} e^{\alpha\left(t-t_{0}\right)} s_{\mathrm{c}}\left(u_{\mathrm{c}}(t), y_{\mathrm{c}}(t)\right) \mathrm{d} t \\
& \quad+\sum_{k \in \mathbb{Z}_{\left[t_{0}, T\right)}} e^{\alpha\left(t_{k}-t_{0}\right)} s_{\mathrm{d}}\left(u_{\mathrm{d}}\left(t_{k}\right), y_{\mathrm{d}}\left(t_{k}\right)\right) \geq 0, \quad T \geq t_{0},\left(u_{\mathrm{c}}, u_{\mathrm{d}}\right) \in u_{\mathrm{c}} \times \boldsymbol{u}_{\mathrm{d}}
\end{aligned}
$$

which, using (3.21), implies that $v_{\mathrm{a}}(0)=0$. Now, it can be easily shown that $v_{\mathrm{a}}(x), x \in \mathscr{D}$, satisfies (3.23) and hence the available storage defined by (3.21) is a storage function 
for $\mathscr{G}$. Finally, it follows from (3.23) that

$$
\begin{aligned}
v_{\mathrm{s}}\left(x\left(t_{0}\right)\right) \geq & e^{\alpha\left(T-t_{0}\right)} v_{\mathrm{s}}(x(T))-\int_{t_{0}}^{T} e^{\alpha\left(t-t_{0}\right)} s_{\mathrm{c}}\left(u_{\mathrm{c}}(t), y_{\mathrm{c}}(t)\right) \mathrm{d} t \\
& -\sum_{k \in \mathbb{Z}_{\left[t_{0}, T\right)}} e^{\alpha\left(t_{k}-t_{0}\right)} s_{\mathrm{d}}\left(u_{\mathrm{d}}\left(t_{k}\right), y_{\mathrm{d}}\left(t_{k}\right)\right) \\
\geq & -\int_{t_{0}}^{T} e^{\alpha\left(t-t_{0}\right)} s_{\mathrm{c}}(u(t), y(t)) \mathrm{d} t \\
& -\sum_{k \in \mathbb{Z}_{\left[t_{0}, T\right)}} e^{\alpha\left(t_{k}-t_{0}\right)} s_{\mathrm{d}}\left(u_{\mathrm{d}}\left(t_{k}\right), y_{\mathrm{d}}\left(t_{k}\right)\right), \quad T \geq t_{0},\left(u_{\mathrm{c}}, u_{\mathrm{d}}\right) \in u_{\mathrm{c}} \times u_{\mathrm{d}},
\end{aligned}
$$

which implies

$$
\begin{aligned}
v_{\mathrm{s}}\left(x\left(t_{0}\right)\right) \geq-\inf _{T \geq t_{0},\left(u_{\mathrm{c}}(\cdot), u_{\mathrm{d}}(\cdot)\right)} & {\left[\int_{t_{0}}^{T} e^{\alpha\left(t-t_{0}\right)} s_{\mathrm{c}}\left(u_{\mathrm{c}}(t), y_{\mathrm{c}}(t)\right) \mathrm{d} t\right.} \\
& \left.+\sum_{k \in \mathbb{Z}_{\left[t_{0}, T\right)}} e^{\alpha\left(t_{k}-t_{0}\right)} s_{\mathrm{d}}\left(u_{\mathrm{d}}\left(t_{k}\right), y_{\mathrm{d}}\left(t_{k}\right)\right)\right] \\
= & v_{\mathrm{a}}\left(x\left(t_{0}\right)\right)
\end{aligned}
$$

and hence (3.22) holds.

Remark 3.6. It follows from Theorem 3.4 that if (3.12) holds for $x\left(t_{0}\right)=0$, then the vector available storage $V_{\mathrm{a}}(x), x \in \mathscr{D}$, is a vector storage function for $\mathscr{G}$. In this case, it follows from Theorem 3.5 that there exists $p \in \overline{\mathbb{R}}_{+}^{q}, p \neq 0$, such that $v_{\mathrm{s}}(x) \triangleq p^{\mathrm{T}} V_{\mathrm{a}}(x)$ is a storage function for $\mathscr{G}$ that satisfies (3.23), and hence by (3.22), $v_{\mathrm{a}}(x) \leq p^{\mathrm{T}} V_{\mathrm{a}}(x), x \in \mathscr{D}$.

Remark 3.7. It is important to note that it follows from Theorem 3.5 that if $\mathscr{G}$ is vector dissipative, then $\mathscr{G}$ can either be (scalar) dissipative or (scalar) exponentially dissipative.

The following theorem provides sufficient conditions guaranteeing that all scalar storage functions defined in terms of vector storage functions, that is, $v_{s}(x)=p^{\mathrm{T}} V_{\mathrm{s}}(x)$, of a given vector dissipative large-scale impulsive nonlinear dynamical system, are positivedefinite. To state this result, the following definition is needed.

Definition 3.8 [10]. A large-scale impulsive dynamical system $\mathscr{G}$ given by (3.1), (3.2), (3.3), and (3.4) is zero-state observable if $\left(u_{\mathrm{c}}(t), u_{\mathrm{d}}\left(t_{k}\right)\right) \equiv(0,0)$ and $\left(y_{\mathrm{c}}(t), y_{\mathrm{d}}\left(t_{k}\right)\right) \equiv(0,0)$ imply $x(t) \equiv 0$.

Theorem 3.9. Consider the large-scale impulsive dynamical system $G$ given by (3.1), (3.2), (3.3), and (3.4) and assume that $\mathscr{G}$ is zero-state observable. Furthermore, assume that $\mathscr{G}$ is vector dissipative (resp., exponentially vector dissipative) with respect to the vector hybrid supply rate $\left(S_{\mathrm{c}}\left(u_{\mathrm{c}}, y_{\mathrm{c}}\right), S_{\mathrm{d}}\left(u_{\mathrm{d}}, y_{\mathrm{d}}\right)\right)$ and there exist $\alpha \geq 0$ and $p \in \mathbb{R}_{+}^{q}$ such that (2.2) holds. In addition, assume that there exist functions $\kappa_{\mathrm{c} i}: \mathscr{y}_{\mathrm{c} i} \rightarrow \boldsymbol{U}_{\mathrm{c} i}$ and $\kappa_{\mathrm{d} i}: \mathscr{y}_{\mathrm{d} i} \rightarrow \boldsymbol{U}_{\mathrm{d} i}$ such that $\kappa_{\mathrm{c} i}(0)=0, \kappa_{\mathrm{d} i}(0)=0, s_{\mathrm{c} i}\left(\kappa_{\mathrm{c} i}\left(y_{\mathrm{c} i}\right), y_{\mathrm{c} i}\right)<0, y_{\mathrm{c} i} \neq 0$, and $s_{\mathrm{d} i}\left(\kappa_{\mathrm{d} i}\left(y_{\mathrm{d} i}\right), y_{\mathrm{d} i}\right)<0, y_{\mathrm{d} i} \neq 0$, for all $i=1, \ldots, q$. Then for all vector storage functions $V_{\mathrm{s}}: \mathscr{D} \rightarrow \overline{\mathbb{R}}_{+}^{q}$, the storage function $v_{\mathrm{s}}(x) \triangleq$ $p^{\mathrm{T}} V_{\mathrm{s}}(x), x \in \mathscr{D}$, is positive-definite; that is, $v_{\mathrm{s}}(0)=0$ and $v_{\mathrm{s}}(x)>0, x \in \mathscr{D}, x \neq 0$. 
Proof. The proof is similar to the proof of [13, Theorem 3.3].

Next, we introduce the concept of vector required supply of a large-scale impulsive dynamical system. Specifically, define the vector required supply of the large-scale impulsive dynamical system $\mathscr{G}$ by

$$
\begin{aligned}
V_{\mathrm{r}}\left(x_{0}\right) \triangleq \inf _{T \leq t_{0},\left(u_{\mathrm{c}}(\cdot), u_{\mathrm{d}}(\cdot)\right)} & {\left[\int_{T}^{t_{0}} e^{-W\left(t-t_{0}\right)} S_{\mathrm{c}}\left(u_{\mathrm{c}}(t), y_{\mathrm{c}}(t)\right) \mathrm{d} t\right.} \\
& \left.+\sum_{k \in \mathbb{Z}_{\left[T, t_{0}\right)}} e^{-W\left(t_{k}-t_{0}\right)} S_{\mathrm{d}}\left(u_{\mathrm{d}}\left(t_{k}\right), y_{\mathrm{d}}\left(t_{k}\right)\right)\right],
\end{aligned}
$$

where $x(t), t \geq T$, is the solution to (3.1), (3.2), (3.3), and (3.4) with $x(T)=0$ and $x\left(t_{0}\right)=x_{0}$. Note that since, with $x\left(t_{0}\right)=0$, the infimum in (3.27) is the zero vector, it follows that $V_{\mathrm{r}}(0)=0$. Moreover, since $\varphi$ is completely reachable, it follows that $V_{\mathrm{r}}(x) \ll \infty$, $x \in \mathscr{D}$. Using the notion of the vector required supply, we present necessary and sufficient conditions for vector dissipativity of a large-scale impulsive dynamical system with respect to a vector hybrid supply rate.

Theorem 3.10. Consider the large-scale impulsive dynamical system $G$ given by (3.1), (3.2), (3.3), and (3.4) and assume that $\mathscr{G}$ is completely reachable. Then $\mathscr{G}$ is vector dissipative (resp., exponentially vector dissipative) with respect to the vector hybrid supply rate $\left(S_{\mathrm{c}}\left(u_{\mathrm{c}}, y_{\mathrm{c}}\right), S_{\mathrm{d}}\left(u_{\mathrm{d}}, y_{\mathrm{d}}\right)\right)$ if and only if

$$
0 \leq \leq V_{\mathrm{r}}(x) \ll \infty, \quad x \in \mathscr{D} .
$$

Moreover, if (3.28) holds, then $V_{\mathrm{r}}(x), x \in \mathscr{D}$, is a vector storage function for $G$. Finally, if the vector available storage $V_{\mathrm{a}}(x), x \in \mathscr{D}$, is a vector storage function for $\mathscr{G}$, then

$$
0 \leq \leq V_{\mathrm{a}}(x) \leq \leq V_{\mathrm{r}}(x) \ll \infty, \quad x \in \mathscr{D} .
$$

Proof. Suppose (3.28) holds and let $x(t), t \in \mathbb{R}$, satisfy (3.1), (3.2), (3.3), and (3.4) with admissible inputs $\left(u_{\mathrm{c}}(t), u_{\mathrm{d}}(t)\right) \in \boldsymbol{U}_{\mathrm{c}} \times \boldsymbol{U}_{\mathrm{d}}, t \in \mathbb{R}$, and $x\left(t_{0}\right)=x_{0}$. Then it follows from the definition of $V_{\mathrm{r}}(\cdot)$ that for $T \leq t_{\mathrm{f}} \leq t_{0}, u_{\mathrm{c}}(\cdot) \in \boldsymbol{U}_{\mathrm{c}}$, and $u_{\mathrm{d}}(\cdot) \in \boldsymbol{u}_{\mathrm{d}}$,

$$
\begin{aligned}
V_{\mathrm{r}}\left(x_{0}\right) \leq & \leq \int_{T}^{t_{0}} e^{-W\left(t-t_{0}\right)} S_{\mathrm{c}}\left(u_{\mathrm{c}}(t), y_{\mathrm{c}}(t)\right) \mathrm{d} t+\sum_{k \in \mathbb{Z}_{\left[T, t_{0}\right)}} e^{-W\left(t_{k}-t_{0}\right)} S_{\mathrm{d}}\left(u_{\mathrm{d}}\left(t_{k}\right), y_{\mathrm{d}}\left(t_{k}\right)\right) \\
= & \int_{T}^{t_{\mathrm{f}}} e^{-W\left(t-t_{0}\right)} S_{\mathrm{c}}\left(u_{\mathrm{c}}(t), y_{\mathrm{c}}(t)\right) \mathrm{d} t+\sum_{k \in \mathbb{Z}_{\left[T, t_{\mathrm{f}}\right)}} e^{-W\left(t_{k}-t_{0}\right)} S_{\mathrm{d}}\left(u_{\mathrm{d}}\left(t_{k}\right), y_{\mathrm{d}}\left(t_{k}\right)\right) \\
& +\int_{t_{\mathrm{f}}}^{t_{0}} e^{-W\left(t-t_{0}\right)} S_{\mathrm{c}}\left(u_{\mathrm{c}}(t), y_{\mathrm{c}}(t)\right) \mathrm{d} t+\sum_{k \in \mathbb{Z}_{\left[\mathrm{t}_{\mathrm{f}}, t_{0}\right)}} e^{-W\left(t_{k}-t_{0}\right)} S_{\mathrm{d}}\left(u_{\mathrm{d}}\left(t_{k}\right), y_{\mathrm{d}}\left(t_{k}\right)\right)
\end{aligned}
$$


and hence,

$$
\begin{aligned}
V_{\mathrm{r}}\left(x_{0}\right) \leq \leq & e^{W\left(t_{0}-t_{\mathrm{f}}\right)} \inf _{T \leq t_{\mathrm{f}},\left(u_{\mathrm{c}}(\cdot), u_{\mathrm{d}}(\cdot)\right)}\left[\int_{T}^{t_{\mathrm{f}}} e^{-W\left(t-t_{\mathrm{f}}\right)} S_{\mathrm{c}}\left(u_{\mathrm{c}}(t), y_{\mathrm{c}}(t)\right) \mathrm{d} t\right. \\
& \left.\quad+\sum_{k \in \mathbb{Z}_{\left[T, t_{\mathrm{f}}\right)}} e^{-W\left(t_{k}-t_{\mathrm{f}}\right)} S_{\mathrm{d}}\left(u_{\mathrm{d}}\left(t_{k}\right), y_{\mathrm{d}}\left(t_{k}\right)\right)\right] \\
& +\int_{t_{\mathrm{f}}}^{t_{0}} e^{-W\left(t-t_{0}\right)} S_{\mathrm{c}}\left(u_{\mathrm{c}}(t), y_{\mathrm{c}}(t)\right) \mathrm{d} t \\
& +\sum_{k \in \mathbb{Z}_{\left[t_{\mathrm{f}}, t_{0}\right)}} e^{-W\left(t_{k}-t_{0}\right)} S_{\mathrm{d}}\left(u_{\mathrm{d}}\left(t_{k}\right), y_{\mathrm{d}}\left(t_{k}\right)\right) \\
= & e^{W\left(t_{0}-t_{\mathrm{f}}\right)} V_{\mathrm{r}}\left(x\left(t_{\mathrm{f}}\right)\right)+\int_{t_{\mathrm{f}}}^{t_{0}} e^{-W\left(t-t_{0}\right)} S_{\mathrm{c}}\left(u_{\mathrm{c}}(t), y_{\mathrm{c}}(t)\right) \mathrm{d} t \\
& +\sum_{k \in \mathbb{Z}_{\left[t_{\mathrm{f}}, t_{0}\right)}} e^{-W\left(t_{k}-t_{0}\right)} S_{\mathrm{d}}\left(u_{\mathrm{d}}\left(t_{k}\right), y_{\mathrm{d}}\left(t_{k}\right)\right)
\end{aligned}
$$

which shows that $V_{\mathrm{r}}(x), x \in \mathscr{D}$, is a vector storage function for $\mathscr{G}_{\text {and hence }} \mathscr{G}_{\text {is vector }}$ dissipative with respect to the vector hybrid supply rate $\left(S_{\mathrm{c}}\left(u_{\mathrm{c}}, y_{\mathrm{c}}\right), S_{\mathrm{d}}\left(u_{\mathrm{d}}, y_{\mathrm{d}}\right)\right)$.

Conversely, suppose that $\mathscr{G}_{\mathcal{S}}$ is vector dissipative with respect to the vector hybrid supply rate $\left(S_{\mathrm{c}}\left(u_{\mathrm{c}}, y_{\mathrm{c}}\right), S_{\mathrm{d}}\left(u_{\mathrm{d}}, y_{\mathrm{d}}\right)\right)$. Then there exists a nonnegative vector storage function $V_{\mathrm{s}}(x)$,

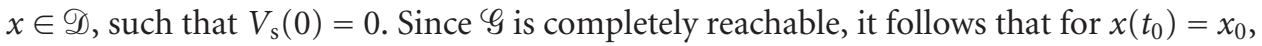
there exist $T<t_{0}$ and $u(t), t \in\left[T, t_{0}\right]$, such that $x(T)=0$. Hence, it follows from the vector hybrid dissipation inequality (3.6) that

$$
\begin{aligned}
0 \leq \leq & V_{\mathrm{s}}\left(x\left(t_{0}\right)\right) \leq \leq e^{W\left(t_{0}-T\right)} V_{\mathrm{s}}(x(T))+\int_{T}^{t_{0}} e^{W\left(t_{0}-t\right)} S_{\mathrm{c}}\left(u_{\mathrm{c}}(t), y_{\mathrm{c}}(t)\right) \mathrm{d} t \\
& +\sum_{k \in \mathbb{Z}_{\left[T, t_{0}\right)}} e^{W\left(t_{0}-t_{k}\right)} S_{\mathrm{d}}\left(u_{\mathrm{d}}\left(t_{k}\right), y_{\mathrm{d}}\left(t_{k}\right)\right)
\end{aligned}
$$

which implies that for all $T \leq t_{0}, u_{\mathrm{c}} \in \mathcal{U}_{\mathrm{c}}$, and $u_{\mathrm{d}} \in \mathcal{U}_{\mathrm{d}}$,

$$
0 \leq \leq \int_{T}^{t_{0}} e^{W\left(t_{0}-t\right)} S_{\mathrm{c}}\left(u_{\mathrm{c}}(t), y_{\mathrm{c}}(t)\right) \mathrm{d} t+\sum_{k \in \mathbb{Z}_{\left[T, t_{0}\right)}} e^{W\left(t_{0}-t_{k}\right)} S_{\mathrm{d}}\left(u_{\mathrm{d}}\left(t_{k}\right), y_{\mathrm{d}}\left(t_{k}\right)\right)
$$

or, equivalently,

$$
\begin{aligned}
0 \leq \leq \inf _{T \leq t_{0},\left(u_{\mathrm{c}}(\cdot), u_{\mathrm{d}}(\cdot)\right)} & {\left[\int_{T}^{t_{0}} e^{W\left(t_{0}-t\right)} S_{\mathrm{c}}\left(u_{\mathrm{c}}(t), y_{\mathrm{c}}(t)\right) \mathrm{d} t\right.} \\
& \left.+\sum_{k \in \mathbb{Z}_{\left[T, t_{0}\right)}} e^{W\left(t_{0}-t_{k}\right)} S_{\mathrm{d}}\left(u_{\mathrm{d}}\left(t_{k}\right), y_{\mathrm{d}}\left(t_{k}\right)\right)\right] \\
=V_{\mathrm{r}}\left(x_{0}\right) . &
\end{aligned}
$$

Since, by complete reachability, $V_{\mathrm{r}}(x) \ll \infty, x \in \mathscr{D}$, it follows that (3.28) holds. 
Finally, suppose that $V_{\mathrm{a}}(x), x \in \mathscr{D}$, is a vector storage function. Then for $x(T)=0$, $x\left(t_{0}\right)=x_{0}, u_{\mathrm{c}} \in \mathcal{U}_{\mathrm{c}}$, and $u_{\mathrm{d}} \in \boldsymbol{U}_{\mathrm{d}}$, it follows that

$$
\begin{aligned}
V_{\mathrm{a}}\left(x\left(t_{0}\right)\right) \leq \leq & e^{W\left(t_{0}-T\right)} V_{\mathrm{a}}(x(T))+\int_{T}^{t_{0}} e^{W\left(t_{0}-t\right)} S_{\mathrm{c}}\left(u_{\mathrm{c}}(t), y_{\mathrm{c}}(t)\right) \mathrm{d} t \\
& +\sum_{k \in \mathbb{Z}_{\left[T, t_{0}\right)}} e^{W\left(t_{0}-t_{k}\right)} S_{\mathrm{d}}\left(u_{\mathrm{d}}\left(t_{k}\right), y_{\mathrm{d}}\left(t_{k}\right)\right),
\end{aligned}
$$

which implies that

$$
\begin{aligned}
0 \leq \leq V_{\mathrm{a}}\left(x\left(t_{0}\right)\right) \leq \leq \inf _{T \leq t_{0},\left(u_{\mathrm{c}}(\cdot), u_{\mathrm{d}}(\cdot)\right)} & {\left[\int_{T}^{t_{0}} e^{W\left(t_{0}-t\right)} S_{\mathrm{c}}\left(u_{\mathrm{c}}(t), y_{\mathrm{c}}(t)\right) \mathrm{d} t\right.} \\
& \left.+\sum_{k \in \mathbb{Z}_{\left[T, t_{0}\right)}} e^{W\left(t_{0}-t_{k}\right)} S_{\mathrm{d}}\left(u_{\mathrm{d}}\left(t_{k}\right), y_{\mathrm{d}}\left(t_{k}\right)\right)\right] \\
=V_{\mathrm{r}}\left(x\left(t_{0}\right)\right), \quad x \in \mathscr{D} . &
\end{aligned}
$$

Since $x\left(t_{0}\right)=x_{0} \in \mathscr{D}$ is arbitrary and, by complete reachability, $V_{\mathrm{r}}(x) \ll \infty, x \in \mathscr{D},(3.36)$ implies (3.29).

The next result is a direct consequence of Theorems 3.4 and 3.10 .

Proposition 3.11. Consider the large-scale impulsive dynamical system $G_{\text {given }}$ by (3.1), (3.2), (3.3), and (3.4). Let $M=\operatorname{diag}\left[\mu_{1}, \ldots, \mu_{q}\right]$ be such that $0 \leq \mu_{i} \leq 1, i=1, \ldots, q$. If $V_{\mathrm{a}}(x)$, $x \in \mathscr{D}$, and $V_{\mathrm{r}}(x), x \in \mathscr{D}$, are vector storage functions for $\mathscr{G}$, then

$$
V_{\mathrm{s}}(x)=M V_{\mathrm{a}}(x)+\left(I_{q}-M\right) V_{\mathrm{r}}(x), \quad x \in \mathscr{D},
$$

is a vector storage function for $\mathscr{G}$.

Next, recall that if $\mathscr{G}$ is vector dissipative (resp., exponentially vector dissipative), then there exist $p \in \overline{\mathbb{R}}_{+}^{q}, p \neq 0$, and $\alpha \geq 0$ (resp., $p \in \mathbb{R}_{+}^{q}$ and $\alpha>0$ ) such that (2.2) and (3.20) hold. Now, define the (scalar) required supply for the large-scale impulsive dynamical system $\mathscr{G}$ by

$$
\begin{aligned}
v_{\mathrm{r}}\left(x_{0}\right) \triangleq \inf _{T \leq t_{0},\left(u_{\mathrm{c}}(\cdot), u_{\mathrm{d}}(\cdot)\right)} & {\left[\int_{T}^{t_{0}} p^{\mathrm{T}} e^{-W\left(t-t_{0}\right)} S_{\mathrm{c}}\left(u_{\mathrm{c}}(t), y_{\mathrm{c}}(t)\right) \mathrm{d} t\right.} \\
& \left.+\sum_{k \in \mathbb{Z}_{\left[T, t_{0}\right)}} e^{-W\left(t_{k}-t_{0}\right)} S_{\mathrm{d}}\left(u_{\mathrm{d}}\left(t_{k}\right), y_{\mathrm{d}}\left(t_{k}\right)\right)\right] \\
=\inf _{T \leq t_{0},\left(u_{\mathrm{c}}(\cdot), u_{\mathrm{d}}(\cdot)\right)}[ & \int_{T}^{t_{0}} e^{\alpha\left(t-t_{0}\right)} s_{\mathrm{c}}\left(u_{\mathrm{c}}(t), y_{\mathrm{c}}(t)\right) \mathrm{d} t \\
& \left.+\sum_{k \in \mathbb{Z}_{\left[T, t_{0}\right)}} e^{\alpha\left(t_{k}-t_{0}\right)} s_{\mathrm{d}}\left(u_{\mathrm{d}}\left(t_{k}\right), y_{\mathrm{d}}\left(t_{k}\right)\right)\right], \quad x_{0} \in \mathscr{D},
\end{aligned}
$$

where $s_{\mathrm{c}}\left(u_{\mathrm{c}}, y_{\mathrm{c}}\right)=p^{\mathrm{T}} S_{\mathrm{c}}\left(u_{\mathrm{c}}, y_{\mathrm{c}}\right), s_{\mathrm{d}}\left(u_{\mathrm{d}}, y_{\mathrm{d}}\right)=p^{\mathrm{T}} S_{\mathrm{d}}\left(u_{\mathrm{d}}, y_{\mathrm{d}}\right)$, and $x(t), t \geq T$, is the solution to (3.1), (3.2), (3.3), and (3.4) with $x(T)=0$ and $x\left(t_{0}\right)=x_{0}$. It follows from (3.38) that 
the required supply of a large-scale impulsive dynamical system is the minimum amount of generalized energy which can be delivered to the large-scale system in order to transfer it from an initial state $x(T)=0$ to a given state $x\left(t_{0}\right)=x_{0}$. Using the same arguments as in the case of the vector required supply, it follows that $v_{\mathrm{r}}(0)=0$ and $v_{\mathrm{r}}(x)<\infty, x \in \mathscr{D}$.

Next, using the notion of required supply, we show that all storage functions of the form $v_{s}(x)=p^{\mathrm{T}} V_{s}(x)$, where $p \in \overline{\mathbb{R}}_{+}^{q}, p \neq 0$, are bounded from above by the required supply and bounded from below by the available storage. Hence, a dissipative large-scale impulsive dynamical system can only deliver to its surroundings a fraction of all of its stored subsystem energies and can only store a fraction of the work done to all of its subsystems.

Corollary 3.12. Consider the large-scale impulsive dynamical system G given by (3.1), (3.2), (3.3), and (3.4). Assume that $G$ is vector dissipative with respect to the vector hybrid supply rate $\left(S_{\mathrm{c}}\left(u_{\mathrm{c}}, y_{\mathrm{c}}\right), S_{\mathrm{d}}\left(u_{\mathrm{d}}, y_{\mathrm{d}}\right)\right)$ and with vector storage function $V_{\mathrm{s}}: \mathscr{D} \rightarrow \overline{\mathbb{R}}_{+}^{q}$. Then $v_{\mathrm{r}}(x)$, $x \in \mathscr{D}$, is a storage function for $\mathscr{G}$. Moreover, if $v_{\mathrm{s}}(x) \triangleq p^{\mathrm{T}} V_{\mathrm{s}}(x), x \in \mathscr{D}$, where $p \in \overline{\mathbb{R}}_{+}^{q}$, $p \neq 0$, then

$$
0 \leq v_{\mathrm{a}}(x) \leq v_{\mathrm{s}}(x) \leq v_{\mathrm{r}}(x)<\infty, \quad x \in \mathscr{D} .
$$

Proof. It follows from Theorem 3.5 that if $\varphi_{G}$ is vector dissipative with respect to the vector hybrid supply rate $\left(S_{\mathrm{c}}\left(u_{\mathrm{c}}, y_{\mathrm{c}}\right), S_{\mathrm{d}}\left(u_{\mathrm{d}}, y_{\mathrm{d}}\right)\right)$ and with a vector storage function $V_{\mathrm{s}}: \mathscr{D} \rightarrow \overline{\mathbb{R}}_{+}^{q}$, then there exists $p \in \overline{\mathbb{R}}_{+}^{q}, p \neq 0$, such that $\varphi_{\mathcal{G}}$ is dissipative with respect to the hybrid supply rate $\left(s_{\mathrm{c}}\left(u_{\mathrm{c}}, y_{\mathrm{c}}\right), s_{\mathrm{d}}\left(u_{\mathrm{d}}, y_{\mathrm{d}}\right)\right)=\left(p^{\mathrm{T}} S_{\mathrm{c}}\left(u_{\mathrm{c}}, y_{\mathrm{c}}\right), p^{\mathrm{T}} S_{\mathrm{d}}\left(u_{\mathrm{d}}, y_{\mathrm{d}}\right)\right)$ and with storage function $v_{\mathrm{s}}(x)=$ $p^{\mathrm{T}} V_{\mathrm{s}}(x), x \in \mathscr{D}$. Hence, it follows from (3.23), with $x(T)=0$ and $x\left(t_{0}\right)=x_{0}$, that

$$
\begin{gathered}
\int_{T}^{t_{0}} e^{\alpha\left(t-t_{0}\right)} s_{\mathrm{c}}\left(u_{\mathrm{c}}(t), y_{\mathrm{c}}(t)\right) \mathrm{d} t+\sum_{k \in \mathbb{Z}_{\left[T, t_{0}\right)}} e^{\alpha\left(t_{k}-t_{0}\right)} s_{\mathrm{d}}\left(u_{\mathrm{d}}\left(t_{k}\right), y_{\mathrm{d}}\left(t_{k}\right)\right) \geq 0, \\
T \leq t_{0},\left(u_{\mathrm{c}}, u_{\mathrm{d}}\right) \in \mathcal{u}_{\mathrm{c}} \times \boldsymbol{U}_{\mathrm{d}},
\end{gathered}
$$

which implies that $v_{\mathrm{r}}\left(x_{0}\right) \geq 0, x_{0} \in \mathscr{D}$. Furthermore, it is easy to see from the definition of required supply that $v_{\mathrm{r}}(x), x \in \mathscr{D}$, satisfies the dissipation inequality (3.23). Hence, $v_{\mathrm{r}}(x)$, $x \in \mathscr{D}$, is a storage function for $\mathscr{G}$. Moreover, it follows from the dissipation inequality (3.23), with $x(T)=0, x\left(t_{0}\right)=x_{0}, u_{\mathrm{c}} \in U_{\mathrm{c}}$, and $u_{\mathrm{d}} \in U_{\mathrm{d}}$, that

$$
\begin{aligned}
e^{\alpha t_{0}} v_{\mathrm{s}}\left(x\left(t_{0}\right)\right) \leq & e^{\alpha T} v_{\mathrm{s}}(x(T))+\int_{T}^{t_{0}} e^{\alpha t_{\mathrm{c}}}\left(u_{\mathrm{c}}(t), y_{\mathrm{c}}(t)\right) \mathrm{d} t \\
& +\sum_{k \in \mathbb{Z}_{\left[T, t_{0}\right)}} e^{\alpha t_{k}} s_{\mathrm{d}}\left(u_{\mathrm{d}}\left(t_{k}\right), y_{\mathrm{d}}\left(t_{k}\right)\right) \\
= & \int_{T}^{t_{0}} e^{\alpha t_{\mathrm{c}}} s_{\mathrm{c}}\left(u_{\mathrm{c}}(t), y_{\mathrm{c}}(t)\right) \mathrm{d} t+\sum_{k \in \mathbb{Z}_{\left[T, t_{0}\right)}} e^{\alpha t_{k}} s_{\mathrm{d}}\left(u_{\mathrm{d}}\left(t_{k}\right), y_{\mathrm{d}}\left(t_{k}\right)\right),
\end{aligned}
$$


which implies that

$$
\begin{aligned}
v_{\mathrm{s}}\left(x\left(t_{0}\right)\right) \leq \inf _{T \leq t_{0},\left(u_{\mathrm{c}}(\cdot), u_{\mathrm{d}}(\cdot)\right)} & {\left[\int_{T}^{t_{0}} e^{\alpha\left(t-t_{0}\right)} s_{\mathrm{c}}\left(u_{\mathrm{c}}(t), y_{\mathrm{c}}(t)\right) \mathrm{d} t\right.} \\
& \left.+\sum_{k \in \mathbb{Z}_{\left[T, t_{0}\right)}} e^{\alpha\left(t_{k}-t_{0}\right)} s_{\mathrm{d}}\left(u_{\mathrm{d}}\left(t_{k}\right), y_{\mathrm{d}}\left(t_{k}\right)\right)\right] \\
= & v_{\mathrm{r}}\left(x\left(t_{0}\right)\right) .
\end{aligned}
$$

Finally, it follows from Theorem 3.5 that $v_{\mathrm{a}}(x), x \in \mathscr{D}$, is a storage function for $\mathscr{G}$ and hence, using (3.22) and (3.42), (3.39) holds.

Remark 3.13. It follows from Theorem 3.10 that if $\varphi$ is vector dissipative with respect to the vector hybrid supply rate $\left(S_{\mathrm{c}}\left(u_{\mathrm{c}}, y_{\mathrm{c}}\right), S_{\mathrm{d}}\left(u_{\mathrm{d}}, y_{\mathrm{d}}\right)\right)$, then $V_{\mathrm{r}}(x), x \in \mathscr{D}$, is a vector storage function for $\varphi$ and, by Theorem 3.5, there exists $p \in \overline{\mathbb{R}}_{+}^{q}, p \neq 0$, such that $v_{\mathrm{s}}(x) \triangleq$ $p^{\mathrm{T}} V_{\mathrm{r}}(x), x \in \mathscr{D}$, is a storage function for $\mathscr{G}$ satisfying (3.23). Hence, it follows from Corollary 3.12 that $p^{\mathrm{T}} V_{\mathrm{r}}(x) \leq v_{\mathrm{r}}(x), x \in \mathscr{D}$.

The next result relates vector (resp., scalar) available storage and vector (resp., scalar) required supply for vector lossless large-scale impulsive dynamical systems.

Theorem 3.14. Consider the large-scale impulsive dynamical system $G$ given by (3.1), (3.2), (3.3), and (3.4). Assume that $G$ is completely reachable to and from the origin. If $\varphi$ is vector lossless with respect to the vector hybrid supply rate $\left(S_{\mathrm{c}}\left(u_{\mathrm{c}}, y_{\mathrm{c}}\right), S_{\mathrm{d}}\left(u_{\mathrm{d}}, y_{\mathrm{d}}\right)\right)$ and $V_{\mathrm{a}}(x), x \in \mathscr{D}$, is a vector storage function, then $V_{\mathrm{a}}(x)=V_{\mathrm{r}}(x), x \in \mathscr{D}$. Moreover, if $V_{\mathrm{s}}(x), x \in \mathscr{D}$, is a vector storage function, then all (scalar) storage functions of the form $v_{\mathrm{s}}(x)=p^{\mathrm{T}} V_{\mathrm{s}}(x), x \in \mathscr{D}$, where $p \in \overline{\mathbb{R}}_{+}^{q}, p \neq 0$, are given by

$$
\begin{aligned}
v_{\mathrm{s}}\left(x_{0}\right) & =v_{\mathrm{a}}\left(x_{0}\right)=v_{\mathrm{r}}\left(x_{0}\right) \\
& =-\int_{t_{0}}^{T} e^{\alpha\left(t-t_{0}\right)} s_{\mathrm{c}}\left(u_{\mathrm{c}}(t), y_{\mathrm{c}}(t)\right) \mathrm{d} t-\sum_{k \in \mathbb{Z}_{\left[t_{0}, T\right)}} e^{\alpha\left(t_{k}-t_{0}\right)} s_{\mathrm{d}}\left(u_{\mathrm{d}}\left(t_{k}\right), y_{\mathrm{d}}\left(t_{k}\right)\right) \\
& =\int_{T^{\prime}}^{t_{0}} e^{\alpha\left(t-t_{0}\right)} s_{\mathrm{c}}\left(u_{\mathrm{c}}(t), y_{\mathrm{c}}(t)\right) \mathrm{d} t+\sum_{k \in \mathbb{Z}_{\left[T^{\prime}, t_{0}\right)}} e^{\alpha\left(t_{k}-t_{0}\right)} s_{\mathrm{d}}\left(u_{\mathrm{d}}\left(t_{k}\right), y_{\mathrm{d}}\left(t_{k}\right)\right),
\end{aligned}
$$

where $x(t), t \geq t_{0}$, is the solution to (3.1), (3.2), (3.3), and (3.4) with $u_{\mathrm{c}} \in u_{\mathrm{c}}, u_{\mathrm{d}} \in u_{\mathrm{d}}$, $x\left(T^{\prime}\right)=0, x(T)=0, x\left(t_{0}\right)=x_{0} \in \mathscr{D}, s_{\mathrm{c}}\left(u_{\mathrm{c}}, y_{\mathrm{c}}\right)=p^{\mathrm{T}} S_{\mathrm{c}}\left(u_{\mathrm{c}}, y_{\mathrm{c}}\right)$, and $s_{\mathrm{d}}\left(u_{\mathrm{d}}, y_{\mathrm{d}}\right)=p^{\mathrm{T}} S_{\mathrm{d}}\left(u_{\mathrm{d}}\right.$, $\left.y_{\mathrm{d}}\right)$.

Proof. The proof is similar to the proof of [13, Theorem 3.5].

The next proposition presents a characterization for vector dissipativity of large-scale impulsive dynamical systems in the case where $V_{\mathrm{s}}(\cdot)$ is continuously differentiable. 
Proposition 3.15. Consider the large-scale impulsive dynamical system $\varphi_{\text {given }}$ by (3.1), (3.2), (3.3), and (3.4) and assume $V_{\mathrm{s}}=\left[v_{\mathrm{s} 1}, \ldots, v_{\mathrm{s} q}\right]^{\mathrm{T}}: \mathscr{D} \rightarrow \overline{\mathbb{R}}_{+}^{q}$ is a continuously differentiable vector storage function for $G_{S}$ and $\varphi_{G}$ is completely reachable. Then $G$ is vector dissipative with respect to the vector hybrid supply rate $\left(S_{\mathrm{c}}\left(u_{\mathrm{c}}, y_{\mathrm{c}}\right), S_{\mathrm{d}}\left(u_{\mathrm{d}}, y_{\mathrm{d}}\right)\right)$ if and only if

$$
\begin{gathered}
\dot{V}_{\mathrm{s}}(x(t)) \leq \leq W V_{\mathrm{s}}(x(t))+S_{\mathrm{c}}\left(u_{\mathrm{c}}(t), y_{\mathrm{c}}(t)\right), \quad t_{k}<t \leq t_{k+1}, \\
V_{\mathrm{s}}\left(x\left(t_{k}\right)+F_{\mathrm{d}}\left(x\left(t_{k}\right), u_{\mathrm{d}}\left(t_{k}\right)\right)\right) \leq \leq V_{\mathrm{s}}\left(x\left(t_{k}\right)\right)+S_{\mathrm{d}}\left(u_{\mathrm{d}}\left(t_{k}\right), y_{\mathrm{d}}\left(t_{k}\right)\right), \quad k \in \overline{\mathbb{Z}}_{+},
\end{gathered}
$$

where $\dot{V}_{\mathrm{s}}(x(t))$ denotes the total time derivative of each component of $V_{\mathrm{s}}(\cdot)$ along the state trajectories $x(t), t_{k}<t \leq t_{k+1}$, of $G_{\text {. }}$

Proof. The proof is similar to the proof of [13, Proposition 3.2].

Recall that if a disconnected subsystem $\mathscr{G}_{i}$ (i.e., $\mathscr{\Phi}_{\mathrm{c} i}(x) \equiv 0$ and $\left.\mathscr{I}_{\mathrm{d} i}(x) \equiv 0, i \in\{1, \ldots, q\}\right)$ of a large-scale impulsive dynamical system $\mathscr{G}$ is exponentially dissipative (resp., dissipative) with respect to a hybrid supply rate $\left(s_{\mathrm{c} i}\left(u_{\mathrm{c} i}, y_{\mathrm{c} i}\right), s_{\mathrm{d} i}\left(u_{\mathrm{d} i}, y_{\mathrm{d} i}\right)\right)$, then there exist a storage function $v_{s i}: \mathbb{R}^{n_{i}} \rightarrow \overline{\mathbb{R}}_{+}$and a constant $\varepsilon_{i}>0$ (resp., $\varepsilon_{i}=0$ ) such that the dissipation inequality (2.11) holds. In the case where $v_{s i}: \mathbb{R}^{n_{i}} \rightarrow \overline{\mathbb{R}}_{+}$is continuously differentiable and $\mathscr{G}$ is completely reachable, $(2.11)$ yields

$$
\begin{gathered}
v_{\mathrm{s} i}^{\prime}\left(x_{i}\right)\left(f_{\mathrm{c} i}\left(x_{i}\right)+G_{\mathrm{c} i}\left(x_{i}\right) u_{\mathrm{c} i}\right) \leq-\varepsilon_{i} v_{\mathrm{s} i}\left(x_{i}\right)+s_{\mathrm{c} i}\left(u_{\mathrm{c} i}, y_{\mathrm{c} i}\right), \quad x \notin \mathscr{L}_{i}, u_{\mathrm{c} i} \in \mathcal{U}_{\mathrm{c} i}, \\
v_{\mathrm{s} i}\left(x_{i}+f_{\mathrm{d} i}\left(x_{i}\right)+G_{\mathrm{d} i}\left(x_{i}\right) u_{\mathrm{d} i}\right) \leq v_{\mathrm{s} i}\left(x_{i}\right)+s_{\mathrm{d} i}\left(u_{\mathrm{d} i}, y_{\mathrm{d} i}\right), \quad x \in \mathscr{L}_{i}, u_{\mathrm{d} i} \in \mathcal{U}_{\mathrm{d} i},
\end{gathered}
$$

where $\mathscr{E}_{i} \triangleq \mathbb{R}^{n_{1}} \times \cdots \times \mathbb{R}^{n_{i-1}} \times \mathscr{E}_{x_{i}} \times \mathbb{R}^{n_{i+1}} \times \cdots \times \mathbb{R}^{q} \subset \mathbb{R}^{n}$ and $\mathscr{E}_{x_{i}} \subset \mathbb{R}^{n_{i}}, i=1, \ldots, q$. The next result relates exponential dissipativity with respect to a scalar hybrid supply rate of each disconnected subsystem $\mathscr{G}_{i}$ of $\mathscr{G}_{\text {with vector dissipativity (or, possibly, exponential }}$ vector dissipativity) of $\mathscr{G}$ with respect to a hybrid vector supply rate.

Proposition 3.16. Consider the large-scale impulsive dynamical system $G_{\text {given }}$ by (3.1), (3.2), (3.3), and (3.4) with $\mathscr{E}_{x}=\cup_{i=1}^{q} \mathscr{L}_{i}$. Assume that $\mathscr{G}$ is completely reachable and each

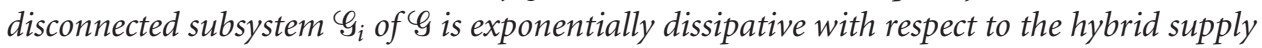
rate $\left(s_{\mathrm{c} i}\left(u_{\mathrm{c} i}, y_{\mathrm{c} i}\right), s_{\mathrm{d} i}\left(u_{\mathrm{d} i}, y_{\mathrm{d} i}\right)\right)$ and with a continuously differentiable storage function $v_{\mathrm{s} i}$ : $\mathbb{R}^{n_{i}} \rightarrow \overline{\mathbb{R}}_{+}, i=1, \ldots, q$. Furthermore, assume that interconnection functions $\mathscr{I}_{\mathrm{c} i}: \mathscr{D} \rightarrow \mathbb{R}^{n_{i}}$ and $\mathscr{I}_{\mathrm{d} i}: \mathscr{D} \rightarrow \mathbb{R}^{n_{i}}, i=1, \ldots, q$, of $\mathscr{G}_{\text {are }}$ such that

$$
\begin{gathered}
v_{\mathrm{s} i}^{\prime}\left(x_{i}\right) \mathscr{I}_{\mathrm{c} i}(x) \leq \sum_{j=1}^{q} \xi_{i j}(x) v_{\mathrm{s} j}\left(x_{j}\right), \quad x \notin \mathscr{E}_{x}, \\
v_{\mathrm{s} i}\left(x_{i}+f_{\mathrm{d} i}\left(x_{i}\right)+\mathscr{I}_{\mathrm{d} i}(x)+G_{\mathrm{d} i}\left(x_{i}\right) u_{\mathrm{d} i}\right) \\
\leq v_{\mathrm{s} i}\left(x_{i}+f_{\mathrm{d} i}\left(x_{i}\right)+G_{\mathrm{d} i}\left(x_{i}\right) u_{\mathrm{d} i}\right), \quad x \in \mathscr{L}_{x}, u_{\mathrm{d} i} \in \mathcal{U}_{\mathrm{d} i}, i=1, \ldots, q,
\end{gathered}
$$


where $\xi_{i j}: \mathscr{D} \rightarrow \mathbb{R}, i, j=1, \ldots, q$, are given bounded functions. If $W \in \mathbb{R}^{q \times q}$ is semistable (resp., asymptotically stable), with

$$
W_{(i, j)}= \begin{cases}-\varepsilon_{i}+\alpha_{i i}, & i=j, \\ \alpha_{i j}, & i \neq j\end{cases}
$$

where $\varepsilon_{i}>0$ and $\alpha_{i j} \triangleq \max \left\{0, \sup _{x \in \mathscr{D}} \xi_{i j}(x)\right\}, i, j=1, \ldots, q$, then $G$ is vector dissipative (respectively, exponentially vector dissipative) with respect to the vector hybrid supply rate $\left(S_{\mathrm{c}}\left(u_{\mathrm{c}}, y_{\mathrm{c}}\right), S_{\mathrm{d}}\left(u_{\mathrm{d}}, y_{\mathrm{d}}\right)\right) \triangleq\left(\left[s_{\mathrm{c} 1}\left(u_{\mathrm{c} 1}, y_{\mathrm{c} 1}\right), \ldots, s_{\mathrm{c} q}\left(u_{\mathrm{c} q}, y_{\mathrm{c} q}\right)\right]^{\mathrm{T}},\left[s_{\mathrm{d} 1}\left(u_{\mathrm{d} 1}, y_{\mathrm{d} 1}\right), \ldots, s_{\mathrm{d} q}\left(u_{\mathrm{d} q}, y_{\mathrm{d} q}\right)\right]^{\mathrm{T}}\right)$ and with vector storage function $V_{\mathrm{s}}(x) \triangleq\left[v_{\mathrm{s} 1}\left(x_{1}\right), \ldots, v_{\mathrm{s} q}\left(x_{q}\right)\right]^{\mathrm{T}}, x \in \mathscr{D}$.

Proof. Since each disconnected impulsive subsystem $\mathscr{G}_{i}$ of $\varphi_{\text {is }}$ exponentially dissipative with respect to the hybrid supply rate $s_{\mathrm{c} i}\left(u_{\mathrm{c} i}, y_{\mathrm{c} i}\right), i=1, \ldots, q$, it follows from (3.45) and (3.46) that, for all $u_{\mathrm{c} i} \in \boldsymbol{U}_{\mathrm{c} i}$ and $i=1, \ldots, q$,

$$
\begin{gathered}
\dot{v}_{\mathrm{s} i}\left(x_{i}(t)\right)=v_{\mathrm{s} i}^{\prime}\left(x_{i}(t)\right)\left[f_{\mathrm{c} i}\left(x_{i}(t)\right)+\Phi_{\mathrm{c} i}(x(t))+G_{\mathrm{c} i}\left(x_{i}(t)\right) u_{\mathrm{c} i}(t)\right] \\
\leq-\varepsilon_{i} v_{\mathrm{s} i}\left(x_{i}(t)\right)+s_{\mathrm{c} i}\left(u_{\mathrm{c} i}(t), y_{\mathrm{c} i}(t)\right)+\sum_{j=1}^{q} \xi_{\mathrm{ij}}(x(t)) v_{\mathrm{s} j}\left(x_{j}(t)\right) \\
\leq-\varepsilon_{i} v_{\mathrm{s} i}\left(x_{i}(t)\right)+s_{\mathrm{c} i}\left(u_{\mathrm{c} i}(t), y_{\mathrm{c} i}(t)\right)+\sum_{j=1}^{q} \alpha_{i j} v_{\mathrm{s} j}\left(x_{j}(t)\right), \quad t_{k}<t \leq t_{k+1}, \\
v_{\mathrm{s} i}\left(x_{i}\left(t_{k}\right)+f_{\mathrm{d} i}\left(x_{i}\left(t_{k}\right)\right)+\Phi_{\mathrm{d} i}\left(x\left(t_{k}\right)\right)+G_{\mathrm{d} i}\left(x_{i}\left(t_{k}\right)\right) u_{\mathrm{d} i}\left(t_{k}\right)\right) \\
\leq v_{\mathrm{s} i}\left(x_{i}\left(t_{k}\right)+f_{\mathrm{d} i}\left(x_{i}\left(t_{k}\right)\right)+G_{\mathrm{d} i}\left(x_{i}\left(t_{k}\right)\right) u_{\mathrm{d} i}\left(t_{k}\right)\right) \\
\leq v_{\mathrm{s} i}\left(x_{i}\left(t_{k}\right)\right)+s_{\mathrm{d} i}\left(u_{\mathrm{d} i}\left(t_{k}\right), y_{\mathrm{d} i}\left(t_{k}\right)\right), \quad k \in \overline{\mathbb{Z}}_{+} .
\end{gathered}
$$

Now, the result follows from Proposition 3.15 by noting that for all subsystems $\mathscr{G}_{i}$ of $\varphi_{\text {, }}$

$$
\begin{gathered}
\dot{V}_{\mathrm{s}}(x(t)) \leq \leq W V_{\mathrm{s}}(x(t))+S_{\mathrm{c}}\left(u_{\mathrm{c}}(t), y_{\mathrm{c}}(t)\right), \quad t_{k}<t \leq t_{k+1}, u_{\mathrm{c}} \in \mathcal{U}_{\mathrm{c}}, \\
V_{\mathrm{s}}\left(x\left(t_{k}\right)+F_{\mathrm{d}}\left(x\left(t_{k}\right), u_{\mathrm{d}}\left(t_{k}\right)\right)\right) \leq \leq V_{\mathrm{s}}\left(x\left(t_{k}\right)\right)+S_{\mathrm{d}}\left(u_{\mathrm{d}}\left(t_{k}\right), y_{\mathrm{d}}\left(t_{k}\right)\right), \quad k \in \overline{\mathbb{Z}}_{+}, u_{\mathrm{d}} \in \boldsymbol{U}_{\mathrm{d}},
\end{gathered}
$$

where $W$ is essentially nonnegative and, by assumption, semistable (resp., asymptotically stable) and $V_{s}(x) \triangleq\left[v_{s 1}\left(x_{1}\right), \ldots, v_{s q}\left(x_{q}\right)\right]^{\mathrm{T}}, x \in \mathscr{D}$, is a vector storage function for $\mathscr{G}$.

\section{Extended Kalman-Yakubovich-Popov conditions for large-scale impulsive dynamical systems}

In this section, we show that vector dissipativeness (resp., exponential vector dissipativeness) of a large-scale impulsive dynamical system $\mathscr{G}$ of the form (3.1), (3.2), (3.3), and (3.4) can be characterized in terms of the local subsystem functions $f_{\mathrm{ci}}(\cdot), G_{\mathrm{ci}}(\cdot), h_{\mathrm{ci}}(\cdot)$, $J_{\mathrm{c} i}(\cdot), f_{\mathrm{d} i}(\cdot), G_{\mathrm{d} i}(\cdot), h_{\mathrm{d} i}(\cdot)$, and $J_{\mathrm{d} i}(\cdot)$, along with the interconnection structures $\Phi_{\mathrm{c} i}(\cdot)$ and $\mathscr{I}_{\mathrm{d} i}(\cdot)$ for $i=1, \ldots, q$. For the results in this section, we consider the special case 
of dissipative systems with quadratic vector hybrid supply rates and set $\mathscr{D}=\mathbb{R}^{n}, \boldsymbol{U}_{\mathrm{c} i}=$ $\mathbb{R}^{m_{\mathrm{c} i}}, \mathscr{U}_{\mathrm{d} i}=\mathbb{R}^{m_{\mathrm{d} i}}, \mathscr{Y}_{\mathrm{c} i}=\mathbb{R}^{l_{\mathrm{c} i}}$, and $\mathscr{Y}_{\mathrm{d} i}=\mathbb{R}^{l_{\mathrm{d} i}}$. Furthermore, we assume that $\mathscr{L}=\mathscr{E}_{x} \times \mathbb{R}^{m_{\mathrm{c}}}$, where $\mathscr{E}_{x} \subset \mathscr{D}$, so that resetting occurs only when $x(t)$ intersects $\mathscr{E}_{x}$. Specifically, let $R_{\mathrm{c} i} \in \mathbb{S}^{m_{\mathrm{c} i}}, S_{\mathrm{c} i} \in \mathbb{R}^{l i \times m_{\mathrm{c} i}}, Q_{\mathrm{c} i} \in \mathbb{S}^{l_{\mathrm{c} i}}, R_{\mathrm{d} i} \in \mathbb{S}^{m_{\mathrm{d} i}}, S_{\mathrm{d} i} \in \mathbb{R}^{l_{\mathrm{d} i} \times m_{\mathrm{d} i}}$, and $Q_{\mathrm{d} i} \in \mathbb{S}^{l_{\mathrm{d} i}}$ be given and assume $S_{\mathrm{c}}\left(u_{\mathrm{c}}, y_{\mathrm{c}}\right)$ is such that $s_{\mathrm{c} i}\left(u_{\mathrm{c} i}, y_{\mathrm{c} i}\right)=y_{\mathrm{c} i}^{\mathrm{T}} Q_{\mathrm{c} i} y_{\mathrm{c} i}+2 y_{\mathrm{c} i}^{\mathrm{T}} S_{\mathrm{c} i} u_{\mathrm{c} i}+u_{\mathrm{c} i}^{\mathrm{T}} R_{\mathrm{c} i} u_{\mathrm{c} i}$ and $S_{\mathrm{d}}\left(u_{\mathrm{d}}, y_{\mathrm{d}}\right)$ is such that $s_{\mathrm{d} i}\left(u_{\mathrm{d} i}, y_{\mathrm{d} i}\right)=y_{\mathrm{d} i}^{\mathrm{T}} Q_{\mathrm{d} i} y_{\mathrm{d} i}+2 y_{\mathrm{d} i}^{\mathrm{T}} S_{\mathrm{d} i} u_{\mathrm{d} i}+u_{\mathrm{d} i}^{\mathrm{T}} R_{\mathrm{d} i} u_{\mathrm{d} i}, i=1, \ldots, q$. Furthermore, for the remainder of this paper, we assume that there exists a continuously differentiable vector storage function $V_{s}(x), x \in \mathbb{R}^{n}$, for the large-scale impulsive dynamical system

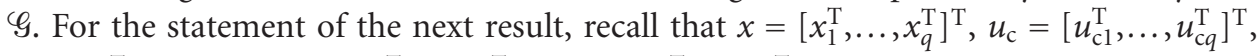
$y_{\mathrm{c}}=\left[y_{\mathrm{c} 1}^{\mathrm{T}}, \ldots, y_{\mathrm{c} q}^{\mathrm{T}}\right]^{\mathrm{T}}, u_{\mathrm{d}}=\left[u_{\mathrm{d} 1}^{\mathrm{T}}, \ldots, u_{\mathrm{d} q}^{\mathrm{T}}\right]^{\mathrm{T}}, y_{\mathrm{d}}=\left[y_{\mathrm{d} 1}^{\mathrm{T}}, \ldots, y_{\mathrm{d} q}^{\mathrm{T}}\right]^{\mathrm{T}}, x_{i} \in \mathbb{R}^{n_{i}}, u_{\mathrm{c} i} \in \mathbb{R}^{m_{\mathrm{c} i}}, y_{\mathrm{c} i} \in \mathbb{R}^{l_{c i}}$, $u_{\mathrm{d} i} \in \mathbb{R}^{m_{\mathrm{d} i}}, y_{\mathrm{d} i} \in \mathbb{R}^{l_{\mathrm{d} i}}, i=1, \ldots, q, \sum_{i=1}^{q} n_{i}=n, \sum_{i=1}^{q} m_{\mathrm{c} i}=m_{\mathrm{c}}, \sum_{i=1}^{q} m_{\mathrm{d} i}=m_{\mathrm{d}}, \sum_{i=1}^{q} l_{\mathrm{c} i}=l_{\mathrm{c}}$, and $\sum_{i=1}^{q} l_{\mathrm{d} i}=l_{\mathrm{d}}$. Furthermore, for (3.1), (3.2), (3.3), and (3.4), define $\mathscr{F}_{\mathrm{c}}: \mathbb{R}^{n} \rightarrow \mathbb{R}^{n}$, $G_{\mathrm{c}}: \mathbb{R}^{n} \rightarrow \mathbb{R}^{n \times m_{\mathrm{c}}}, h_{\mathrm{c}}: \mathbb{R}^{n} \rightarrow \mathbb{R}^{l_{\mathrm{c}}}, J_{\mathrm{c}}: \mathbb{R}^{n} \rightarrow \mathbb{R}^{l_{\mathrm{c}} \times m_{\mathrm{c}}}, \mathscr{F}_{\mathrm{d}}: \mathbb{R}^{n} \rightarrow \mathbb{R}^{n}, G_{\mathrm{d}}: \mathbb{R}^{n} \rightarrow \mathbb{R}^{n \times m_{\mathrm{d}}}, h_{\mathrm{d}}:$ $\mathbb{R}^{n} \rightarrow \mathbb{R}^{l_{\mathrm{d}}}$, and $J_{\mathrm{d}}: \mathbb{R}^{n} \rightarrow \mathbb{R}^{l_{\mathrm{d}} \times m_{\mathrm{d}}}$ by $\mathscr{F}_{\mathrm{c}}(x) \triangleq\left[\mathscr{F}_{\mathrm{c} 1}^{\mathrm{T}}(x), \ldots, \mathscr{F}_{\mathrm{c} q}^{\mathrm{T}}(x)\right]^{\mathrm{T}}, \mathscr{F}_{\mathrm{d}}(x) \triangleq\left[\mathscr{F}_{\mathrm{d} 1}^{\mathrm{T}}(x), \ldots\right.$, $\left.\mathscr{F}_{\mathrm{d} q}^{\mathrm{T}}(x)\right]^{\mathrm{T}}$, where $\mathscr{F}_{\mathrm{c} i}(x) \triangleq f_{\mathrm{c} i}\left(x_{i}\right)+\mathscr{I}_{\mathrm{c} i}(x), \mathscr{F}_{\mathrm{d} i}(x) \triangleq f_{\mathrm{d} i}\left(x_{i}\right)+\mathscr{I}_{\mathrm{d} i}(x), i=1, \ldots, q, G_{\mathrm{c}}(x) \triangleq$ $\operatorname{diag}\left[G_{\mathrm{c} 1}\left(x_{1}\right), \ldots, G_{\mathrm{c} q}\left(x_{q}\right)\right], \quad G_{\mathrm{d}}(x) \triangleq \operatorname{diag}\left[G_{\mathrm{d} 1}\left(x_{1}\right), \ldots, G_{\mathrm{d} q}\left(x_{q}\right)\right], \quad h_{\mathrm{c}}(x) \triangleq\left[h_{\mathrm{c} 1}^{\mathrm{T}}\left(x_{1}\right), \ldots\right.$, $\left.h_{\mathrm{c} q}^{\mathrm{T}}\left(x_{q}\right)\right]^{\mathrm{T}}, h_{\mathrm{d}}(x) \triangleq\left[h_{\mathrm{d} 1}^{\mathrm{T}}\left(x_{1}\right), \ldots, h_{\mathrm{d} q}^{\mathrm{T}}\left(x_{q}\right)\right]^{\mathrm{T}}, J_{\mathrm{c}}(x) \triangleq \operatorname{diag}\left[J_{\mathrm{c} 1}\left(x_{1}\right), \ldots, J_{\mathrm{c} q}\left(x_{q}\right)\right]$, and $J_{\mathrm{d}}(x) \triangleq$ $\operatorname{diag}\left[J_{\mathrm{d} 1}\left(x_{1}\right), \ldots, J_{\mathrm{d} q}\left(x_{q}\right)\right]$. Moreover, for all $i=1, \ldots, q$, define $\hat{R}_{\mathrm{c} i} \in \mathbb{S}^{m_{\mathrm{c}}}, \hat{S}_{\mathrm{c} i} \in \mathbb{R}^{l_{\mathrm{c}} \times m_{\mathrm{c}}}, \hat{Q}_{\mathrm{c} i} \in$ $\mathbb{S}^{l_{\mathrm{c}}}, \hat{R}_{\mathrm{d} i} \in \mathbb{S}^{m_{\mathrm{d}}}, \hat{S}_{\mathrm{d} i} \in \mathbb{R}^{l_{\mathrm{d}} \times m_{\mathrm{d}}}$, and $\hat{Q}_{\mathrm{d} i} \in \mathbb{S}^{l_{\mathrm{d}}}$ such that each of these block matrices consists of zero blocks except for, respectively, the matrix blocks $R_{\mathrm{c} i} \in \mathbb{S}^{m_{\mathrm{c} i}}, S_{\mathrm{c} i} \in \mathbb{R}^{l_{\mathrm{c} i} \times m_{\mathrm{c} i}}, Q_{\mathrm{c} i} \in \mathbb{S}^{l_{\mathrm{c} i}}$, $R_{\mathrm{d} i} \in \mathbb{S}^{m_{\mathrm{d} i}}, S_{\mathrm{d} i} \in \mathbb{R}^{l_{\mathrm{d} i} \times m_{\mathrm{d} i}}$, and $Q_{\mathrm{d} i} \in \mathbb{S}^{l_{\mathrm{d} i}}$ on $(i, i)$ position. Finally, we introduce a more general definition of vector dissipativity involving an underlying nonlinear comparison system.

Definition 4.1. The large-scale impulsive dynamical system $\mathscr{G}$ given by (3.1), (3.2), (3.3), and (3.4) is vector dissipative (resp., exponentially vector dissipative) with respect to the vector hybrid supply rate $\left(S_{\mathrm{c}}\left(u_{\mathrm{c}}, y_{\mathrm{c}}\right), S_{\mathrm{d}}\left(u_{\mathrm{d}}, y_{\mathrm{d}}\right)\right)$ if there exist a continuous, nonnegativedefinite vector function $V_{\mathrm{s}}=\left[v_{\mathrm{s} 1}, \ldots, v_{\mathrm{s} q}\right]^{\mathrm{T}}: \mathscr{D} \rightarrow \overline{\mathbb{R}}_{+}^{q}$, called a vector storage function, and a class $\mathcal{W}$ function $w_{\mathrm{c}}: \overline{\mathbb{R}}_{+}^{q} \rightarrow \mathbb{R}^{q}$ such that $V_{\mathrm{s}}(0)=0, w_{\mathrm{c}}(0)=0$, the zero solution $r(t) \equiv 0$ to the comparison system (2.6) is Lyapunov (resp., asymptotically) stable, and the vector hybrid dissipation inequality

$$
\begin{aligned}
V_{s}(x(T)) \leq \leq & V_{s}\left(x\left(t_{0}\right)\right)+\int_{t_{0}}^{T} w_{\mathrm{c}}\left(V_{\mathrm{s}}(x(t))\right) \mathrm{d} t+\int_{t_{0}}^{T} S_{\mathrm{c}}\left(u_{\mathrm{c}}(t), y_{\mathrm{c}}(t)\right) \mathrm{d} t \\
& +\sum_{k \in \mathbb{Z}_{\left[t_{0}, T\right)}} S_{\mathrm{d}}\left(u_{\mathrm{d}}\left(t_{k}\right), y_{\mathrm{d}}\left(t_{k}\right)\right), \quad T \geq t_{0},
\end{aligned}
$$

is satisfied, where $x(t), t \geq t_{0}$, is the solution to (3.1), (3.2), (3.3), and (3.4) with $u_{\mathrm{c}} \in \boldsymbol{U}_{\mathrm{c}}$

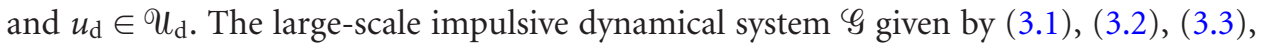
and (3.4) is vector lossless with respect to the vector hybrid supply rate $\left(S_{\mathrm{c}}\left(u_{\mathrm{c}}, y_{\mathrm{c}}\right), S_{\mathrm{d}}\left(u_{\mathrm{d}}, y_{\mathrm{d}}\right)\right)$ if the vector hybrid dissipation inequality is satisfied as an equality with the zero solution $r(t) \equiv 0$ to (2.6) being Lyapunov stable. 
Remark 4.2. If $\mathscr{G}$ is completely reachable and $V_{s}(\cdot)$ is continuously differentiable, then (4.1) can be equivalently written as

$$
\begin{gathered}
\dot{V}_{\mathrm{s}}(x(t)) \leq \leq w_{\mathrm{c}}\left(V_{\mathrm{s}}(x(t))\right)+S_{\mathrm{c}}\left(u_{\mathrm{c}}(t), y_{\mathrm{c}}(t)\right), \quad t_{k}<t \leq t_{k+1}, \\
V_{\mathrm{s}}\left(x\left(t_{k}\right)+F_{\mathrm{d}}\left(x\left(t_{k}\right), u_{\mathrm{d}}\left(t_{k}\right)\right)\right) \leq \leq V_{\mathrm{s}}\left(x\left(t_{k}\right)\right)+S_{\mathrm{d}}\left(u_{\mathrm{d}}\left(t_{k}\right), y_{\mathrm{d}}\left(t_{k}\right)\right), \quad k \in \overline{\mathbb{Z}}_{+},
\end{gathered}
$$

with $u_{\mathrm{c}} \in u_{\mathrm{c}}$ and $u_{\mathrm{d}} \in \boldsymbol{U}_{\mathrm{d}}$.

Remark 4.3. If in Definition 4.1, the function $w_{\mathrm{c}}: \overline{\mathbb{R}}_{+}^{q} \rightarrow \mathbb{R}^{q}$ is such that $w_{\mathrm{c}}(r)=W r$, where $W \in \mathbb{R}^{q \times q}$, then $W$ is essentially nonnegative and Definition 4.1 collapses to Definition 3.2.

Theorem 4.4. Consider the large-scale impulsive dynamical system $G_{\text {given }}$ by (3.1), (3.2), (3.3), and (3.4). Let $R_{\mathrm{c} i} \in \mathbb{S}^{m_{\mathrm{c} i}}, S_{\mathrm{c} i} \in \mathbb{R}^{l_{c i} \times m_{\mathrm{c} i}}, Q_{\mathrm{c} i} \in \mathbb{S}^{l_{c i}}, R_{\mathrm{d} i} \in \mathbb{S}^{m_{\mathrm{d} i}}, S_{\mathrm{d} i} \in \mathbb{R}^{l_{\mathrm{di}} \times m_{\mathrm{d} i}}$, and $Q_{\mathrm{d} i} \in$ $\mathbb{S}^{l_{\mathrm{d} i}}, i=1, \ldots, q$. Then $G$ is vector dissipative (resp., exponentially vector dissipative) with respect to the quadratic hybrid supply rate $\left(S_{\mathrm{c}}\left(u_{\mathrm{c}}, y_{\mathrm{c}}\right), S_{\mathrm{d}}\left(u_{\mathrm{d}}, y_{\mathrm{d}}\right)\right)$, where $s_{\mathrm{c} i}\left(u_{\mathrm{c} i}, y_{\mathrm{c} i}\right)=$ $y_{\mathrm{c} i}^{\mathrm{T}} Q_{\mathrm{c} i} y_{\mathrm{c} i}+2 y_{\mathrm{c} i}^{\mathrm{T}} S_{\mathrm{c} i} u_{\mathrm{c} i}+u_{\mathrm{c} i}^{\mathrm{T}} R_{\mathrm{c} i} u_{\mathrm{c} i}$ and $s_{\mathrm{d} i}\left(u_{\mathrm{d} i}, y_{\mathrm{d} i}\right)=y_{\mathrm{d} i}^{\mathrm{T}} Q_{\mathrm{d} i} y_{\mathrm{d} i}+2 y_{\mathrm{d} i}^{\mathrm{T}} S_{\mathrm{d} i} u_{\mathrm{d} i}+u_{\mathrm{d} i}^{\mathrm{T}} R_{\mathrm{d} i} u_{\mathrm{d} i}, \quad i=$ $1, \ldots, q$, if there exist functions $V_{\mathrm{s}}=\left[v_{\mathrm{s} 1}, \ldots, v_{\mathrm{s} q}\right]^{\mathrm{T}}: \mathbb{R}^{n} \rightarrow \overline{\mathbb{R}}_{+}^{q}, w_{\mathrm{c}}=\left[w_{\mathrm{c} 1}, \ldots, w_{\mathrm{c} q}\right]^{\mathrm{T}}: \overline{\mathbb{R}}_{+}^{q} \rightarrow$ $\mathbb{R}^{q}, \ell_{\mathrm{c} i}: \mathbb{R}^{n} \rightarrow \mathbb{R}^{s_{\mathrm{c} i}}, \mathscr{L}_{\mathrm{c} i}: \mathbb{R}^{n} \rightarrow \mathbb{R}^{s_{\mathrm{c} i} \times m_{\mathrm{c}}}, \ell_{\mathrm{d} i}: \mathbb{R}^{n} \rightarrow \mathbb{R}^{s_{\mathrm{d} i}}, \mathscr{L}_{\mathrm{d} i}: \mathbb{R}^{n} \rightarrow \mathbb{R}^{s_{\mathrm{d} i} \times m_{\mathrm{d}}}, P_{1 i}: \mathbb{R}^{n} \rightarrow \mathbb{R}^{1 \times m_{\mathrm{d}}}$, and $P_{2 i}: \mathbb{R}^{n} \rightarrow \mathbb{N}^{m_{\mathrm{d}}}$ such that $v_{\mathrm{si}}(\cdot)$ is continuously differentiable, $v_{\mathrm{si}}(0)=0, i=1, \ldots, q$, $w_{\mathrm{c}} \in \mathcal{W}, w_{\mathrm{c}}(0)=0$, the zero solution $r(t) \equiv 0$ to (2.6) is Lyapunov (resp., asymptotically) stable,

$$
\begin{aligned}
v_{\mathrm{s} i}(x+ & \left.\mathscr{F}_{\mathrm{d}}(x)+G_{\mathrm{d}}(x) u_{\mathrm{d}}\right) \\
& =v_{\mathrm{s} i}\left(x+\mathscr{F}_{\mathrm{d}}(x)\right)+P_{1 i}(x) u_{\mathrm{d}}+u_{\mathrm{d}}^{\mathrm{T}} P_{2 i}(x) u_{\mathrm{d}}, \quad x \in \mathscr{E}_{x}, u_{\mathrm{d}} \in \mathbb{R}^{m_{\mathrm{d}}},
\end{aligned}
$$

and, for all $i=1, \ldots, q$,

$$
\begin{aligned}
& 0=v_{\mathrm{s} i}^{\prime}(x) \mathscr{F}_{\mathrm{c}}(x)-h_{\mathrm{c}}^{\mathrm{T}}(x) \hat{Q}_{\mathrm{c} i} h_{\mathrm{c}}(x)-w_{\mathrm{c} i}\left(V_{\mathrm{s}}(x)\right)+\ell_{\mathrm{c} i}^{\mathrm{T}}(x) \ell_{\mathrm{c} i}(x), \quad x \notin \mathscr{E}_{x}, \\
& 0=\frac{1}{2} v_{\mathrm{s} i}^{\prime}(x) G_{\mathrm{c}}(x)-h_{\mathrm{c}}^{\mathrm{T}}(x)\left(\hat{S}_{\mathrm{c} i}+\hat{Q}_{\mathrm{c} i} J_{\mathrm{c}}(x)\right)+\ell_{\mathrm{c} i}^{\mathrm{T}}(x) \mathscr{E}_{\mathrm{c} i}(x), \quad x \notin \mathscr{E}_{x}, \\
& 0=\hat{R}_{\mathrm{c} i}+J_{\mathrm{c}}^{\mathrm{T}}(x) \hat{S}_{\mathrm{c} i}+\hat{S}_{\mathrm{c} i}^{\mathrm{T}} J_{\mathrm{c}}(x)+J_{\mathrm{c}}^{\mathrm{T}}(x) \hat{Q}_{\mathrm{c} i} J_{\mathrm{c}}(x)-\mathscr{E}_{\mathrm{c} i}^{\mathrm{T}}(x) \mathscr{E}_{\mathrm{c} i}(x), \quad x \notin \mathscr{E}_{x}, \\
& 0=v_{\mathrm{s} i}\left(x+\mathscr{F}_{\mathrm{d}}(x)\right)-h_{\mathrm{d}}^{\mathrm{T}}(x) \hat{Q}_{\mathrm{d} i} h_{\mathrm{d}}(x)-v_{\mathrm{s} i}(x)+\ell_{\mathrm{d} i}^{\mathrm{T}}(x) \ell_{\mathrm{d} i}(x), \quad x \in \mathscr{E}_{x}, \\
& 0=\frac{1}{2} P_{1 i}(x)-h_{\mathrm{d}}^{\mathrm{T}}(x)\left(\hat{S}_{\mathrm{d} i}+\hat{Q}_{\mathrm{d} i} J_{\mathrm{d}}(x)\right)+\ell_{\mathrm{d} i}^{\mathrm{T}}(x) \mathscr{E}_{\mathrm{d} i}(x), \quad x \in \mathscr{E}_{x}, \\
& 0=\hat{R}_{\mathrm{d} i}+J_{\mathrm{d}}^{\mathrm{T}}(x) \hat{S}_{\mathrm{d} i}+\hat{S}_{\mathrm{d} i}^{\mathrm{T}} J_{\mathrm{d}}(x)+J_{\mathrm{d}}^{\mathrm{T}}(x) \hat{Q}_{\mathrm{d} i} J_{\mathrm{d}}(x)-P_{2 i}(x)-\mathscr{E}_{\mathrm{d} i}^{\mathrm{T}}(x) \mathscr{E}_{\mathrm{d} i}(x), \quad x \in \mathscr{E}_{x} .
\end{aligned}
$$

Proof. Suppose that there exist functions $v_{s i}: \mathbb{R}^{n} \rightarrow \overline{\mathbb{R}}_{+}, \ell_{\mathrm{ci}}: \mathbb{R}^{n} \rightarrow \mathbb{R}^{s_{c i}}, \mathscr{Z}_{\mathrm{ci}}: \mathbb{R}^{n} \rightarrow \mathbb{R}^{s_{c i} \times m_{\mathrm{c}}}$, $\ell_{\mathrm{d} i}: \mathbb{R}^{n} \rightarrow \mathbb{R}^{s_{\mathrm{d} i}}, \mathscr{L}_{\mathrm{d} i}: \mathbb{R}^{n} \rightarrow \mathbb{R}^{s_{\mathrm{d} i} \times m_{\mathrm{d}}}, w_{\mathrm{c}}: \overline{\mathbb{R}}_{+}^{q} \rightarrow \mathbb{R}^{q}, P_{1 i}: \mathbb{R}^{n} \rightarrow \mathbb{R}^{1 \times m_{\mathrm{d}}}$, and $P_{2 i}: \mathbb{R}^{n} \rightarrow \mathbb{N}^{m_{\mathrm{d}}}$ such that $v_{s i}(\cdot)$ is continuously differentiable and nonnegative-definite, $v_{\mathrm{s} i}(0)=0, i=$ $1, \ldots, q, w_{\mathrm{c}}(0)=0, w_{\mathrm{c}} \in \mathcal{W}$, the zero solution $r(t) \equiv 0$ to (2.6) is Lyapunov (resp., asymptotically) stable, and (4.3), (4.4), (4.5), (4.6), (4.7), (4.8), and (4.9) are satisfied. Then for any $u_{\mathrm{c}} \in \mathcal{U}_{\mathrm{c}}, t, \hat{t} \in \mathbb{R}, t_{k}<t \leq \hat{t} \leq t_{k+1}, k \in \overline{\mathbb{Z}}_{+}$, and $i=1, \ldots, q$, it follows from (4.4), (4.5), 
250 Vector dissipativity and large-scale impulsive systems

and (4.6) that

$$
\begin{gathered}
\int_{t}^{\hat{t}} s_{\mathrm{c} i}\left(u_{\mathrm{c} i}(\sigma), y_{\mathrm{c} i}(\sigma)\right) \mathrm{d} \sigma \\
=\int_{t}^{\hat{t}}\left[u_{\mathrm{c}}^{\mathrm{T}}(\sigma) \hat{R}_{\mathrm{c} i} u_{\mathrm{c}}(\sigma)+2 y_{\mathrm{c}}^{\mathrm{T}}(\sigma) \hat{S}_{\mathrm{c} i} u_{\mathrm{c}}(\sigma)+y_{\mathrm{c}}^{\mathrm{T}}(\sigma) \hat{Q}_{\mathrm{c} i} y_{\mathrm{c}}(\sigma)\right] \mathrm{d} \sigma \\
=\int_{t}^{\hat{t}}\left[h_{\mathrm{c}}^{\mathrm{T}}(x(\sigma)) \hat{Q}_{\mathrm{c} i} h_{\mathrm{c}}(x(\sigma))+2 h_{\mathrm{c}}^{\mathrm{T}}(x(\sigma))\left(\hat{S}_{\mathrm{c} i}+\hat{Q}_{\mathrm{c} i} J_{\mathrm{c}}(x(\sigma))\right) u_{\mathrm{c}}(\sigma)\right. \\
\quad+u_{\mathrm{c}}^{\mathrm{T}}(\sigma)\left(J_{\mathrm{c}}^{\mathrm{T}}(x(\sigma)) \hat{Q}_{\mathrm{c} i} J_{\mathrm{c}}(x(\sigma))+J_{\mathrm{c}}^{\mathrm{T}}(x(\sigma)) \hat{S}_{\mathrm{c} i}\right. \\
\left.\left.\quad+\hat{S}_{\mathrm{c} i}^{\mathrm{T}} J_{\mathrm{c}}(x(\sigma))+\hat{R}_{\mathrm{c} i}\right) u_{\mathrm{c}}(\sigma)\right] \mathrm{d} \sigma \\
=\int_{t}^{\hat{t}}\left[v_{\mathrm{s} i}^{\prime}(x(\sigma))\left(\mathscr{F}_{\mathrm{c}}(x(\sigma))+G_{\mathrm{c}}(x(\sigma)) u_{\mathrm{c}}(\sigma)\right)+\ell_{\mathrm{c} i}^{\mathrm{T}}(x(\sigma)) \ell_{\mathrm{c} i}(x(\sigma))\right. \\
\quad+2 \ell_{\mathrm{c} i}^{\mathrm{T}}(x(\sigma)) \mathscr{L}_{\mathrm{c} i}(x(\sigma)) u_{\mathrm{c}}(\sigma) \\
\left.\quad+u_{\mathrm{c}}^{\mathrm{T}}(\sigma) \mathscr{E}_{\mathrm{c} i}^{\mathrm{T}}(x(\sigma)) \mathscr{E}_{\mathrm{c} i}(x(\sigma)) u_{\mathrm{c}}(\sigma)-w_{\mathrm{c} i}\left(V_{\mathrm{s}}(x(\sigma))\right)\right] \mathrm{d} \sigma \\
=\int_{t}^{\hat{t}}\left[\dot{v}_{\mathrm{s} i}(x(\sigma))+\left[\ell_{\mathrm{c} i}(x(\sigma))+\mathscr{L}_{\mathrm{c} i}(x(\sigma)) u_{\mathrm{c}}(\sigma)\right]^{\mathrm{T}}\right. \\
\left.\quad \times\left[\ell_{\mathrm{c} i}(x(\sigma))+\mathscr{E _ { c } i}(x(\sigma)) u_{\mathrm{c}}(\sigma)\right]-w_{\mathrm{c} i}\left(V_{\mathrm{s}}(x(\sigma))\right)\right] \mathrm{d} \sigma \\
\geq v_{\mathrm{s} i}(x(\hat{t}))-v_{\mathrm{s} i}(x(t))-\int_{t}^{\hat{t}} w_{\mathrm{c} i}\left(V_{\mathrm{s}}(x(\sigma))\right) \mathrm{d} \sigma,
\end{gathered}
$$

where $x(\sigma), \sigma \in\left(t_{k}, t_{k+1}\right]$, satisfies (3.1). Next, for any $u_{\mathrm{d}} \in \mathbb{R}^{m_{\mathrm{d}}}, t_{k} \in \mathbb{R}$, and $k \in \overline{\mathbb{Z}}_{+}$, it follows from (4.3), (4.7), (4.8), and (4.9) that

$$
\begin{aligned}
v_{\mathrm{s} i}(x+ & \left.\mathscr{F}_{\mathrm{d}}(x)+G_{\mathrm{d}}(x) u_{\mathrm{d}}\right)-v_{\mathrm{s} i}(x) \\
= & v_{\mathrm{s} i}\left(x+\mathscr{F}_{\mathrm{d}}(x)\right)-v_{\mathrm{s} i}(x)+P_{1 i}(x) u_{\mathrm{d}}+u_{\mathrm{d}}^{\mathrm{T}} P_{2 i}(x) u_{\mathrm{d}} \\
= & h_{\mathrm{d}}^{\mathrm{T}}(x) \hat{Q}_{\mathrm{d} i} h_{\mathrm{d}}(x)-\ell_{\mathrm{d} i}^{\mathrm{T}}(x) \ell_{\mathrm{d} i}(x) \\
& +2\left[h_{\mathrm{d}}^{\mathrm{T}}(x)\left(\hat{Q}_{\mathrm{d} i} J_{\mathrm{d}}(x)+\hat{S}_{\mathrm{d} i}\right)-\ell_{\mathrm{d} i}^{\mathrm{T}}(x) \mathscr{L}_{\mathrm{d} i}(x)\right] u_{\mathrm{d}} \\
& +u_{\mathrm{d}}^{\mathrm{T}}\left[\hat{R}_{\mathrm{d} i}+\hat{S}_{\mathrm{d} i}^{\mathrm{T}} J_{\mathrm{d}}(x)+J_{\mathrm{d}}^{\mathrm{T}}(x) \hat{S}_{\mathrm{d} i}+J_{\mathrm{d}}^{\mathrm{T}}(x) \hat{Q}_{\mathrm{d} i} J_{\mathrm{d}}(x)-\mathscr{L}_{\mathrm{d} i}^{\mathrm{T}}(x) \mathscr{E}_{\mathrm{d} i}(x)\right] u_{\mathrm{d}} \\
= & s_{\mathrm{d} i}\left(u_{\mathrm{d} i}, y_{\mathrm{d} i}\right)-\left[\ell_{\mathrm{d} i}(x)+\mathscr{L}_{\mathrm{d} i}(x) u_{\mathrm{d}}\right]^{\mathrm{T}}\left[\ell_{\mathrm{d} i}(x)+\mathscr{E}_{\mathrm{d} i}(x) u_{\mathrm{d}}\right] \\
\leq & s_{\mathrm{d} i}\left(u_{\mathrm{d} i}, y_{\mathrm{d} i}\right) .
\end{aligned}
$$

Now, using (4.10) and (4.11), the result is immediate from Remark 4.2 with vector storage function $V_{\mathrm{s}}(x)=\left[v_{\mathrm{s} 1}(x), \ldots, v_{\mathrm{s} q}(x)\right]^{\mathrm{T}}, x \in \mathbb{R}^{n}$. 
Using (4.4), (4.5), (4.6), (4.7), (4.8), and (4.9), it follows that for $T \geq t_{0} \geq 0, k \in \mathbb{Z}_{\left[t_{0}, T\right)}$, and $i=1, \ldots, q$,

$$
\begin{aligned}
\int_{t_{0}}^{T} s_{c i}( & \left.u_{c i}(t), y_{c i}(t)\right) \mathrm{d} t+\int_{t_{0}}^{T} w_{\mathrm{c} i}\left(V_{\mathrm{s}}(x(t))\right) \mathrm{d} t+\sum_{k \in \mathbb{Z}_{\left[t_{0}, T\right)}} s_{\mathrm{d} i}\left(u_{\mathrm{d}}\left(t_{k}\right), y_{\mathrm{d}}\left(t_{k}\right)\right) \\
= & v_{\mathrm{si}}(x(T))-v_{\mathrm{si}}\left(x\left(t_{0}\right)\right) \\
& +\int_{t_{0}}^{T}\left[\ell_{\mathrm{c} i}(x(t))+\mathscr{E}_{\mathrm{ci}}(x(t)) u_{\mathrm{c}}(t)\right]^{\mathrm{T}}\left[\ell_{\mathrm{c} i}(x(t))+\mathscr{E}_{\mathrm{c} i}(x(t)) u_{\mathrm{c}}(t)\right] \mathrm{d} t \\
& +\sum_{k \in \mathbb{Z}_{\left[t_{0}, T\right)}}\left[\ell_{\mathrm{d} i}\left(x\left(t_{k}\right)\right)+\mathscr{L}_{\mathrm{d} i}\left(x\left(t_{k}\right)\right) u_{\mathrm{d}}\left(t_{k}\right)\right]^{\mathrm{T}}\left[\ell_{\mathrm{d} i}\left(x\left(t_{k}\right)\right)+\mathscr{L}_{\mathrm{d} i}\left(x\left(t_{k}\right)\right) u_{\mathrm{d}}\left(t_{k}\right)\right],
\end{aligned}
$$

where $V_{\mathrm{s}}(x)=\left[v_{\mathrm{s} 1}(x), \ldots, v_{\mathrm{s} q}(x)\right]^{\mathrm{T}}, x \in \mathbb{R}^{n}$, which can be interpreted as a generalized energy balance equation for the ith impulsive subsystem of $\mathscr{G}$, where $v_{s i}(x(T))-v_{s i}\left(x\left(t_{0}\right)\right)$ is the stored or accumulated generalized energy of the $i$ th impulsive subsystem, the two path dependent terms on the left-hand side are, respectively, the external supplied energy to the $i$ th subsystem over the continuous-time dynamics and the energy gained over the continuous-time dynamics by the ith subsystem from the net energy flow between all subsystems due to subsystem coupling, the last discrete term on the left-hand side corresponds to the external supplied energy to the ith subsystem at the resetting instants, the second path-dependent term on the right-hand side corresponds to the dissipated energy from the $i$ th impulsive subsystem over the continuous-time dynamics, and the last discrete term on the right-hand side corresponds to the dissipated energy from the $i$ th impulsive subsystem at the resetting instants. Equivalently, (4.12) can be rewritten as

$$
\begin{aligned}
& \dot{v}_{\mathrm{si}}(x(t))=s_{\mathrm{ci}}\left(u_{\mathrm{c} i}(t), y_{\mathrm{c} i}(t)\right)+w_{\mathrm{c} i}\left(V_{\mathrm{s}}(x(t))\right) \\
&-\left[\ell_{\mathrm{c} i}(x(t))+\mathscr{L}_{\mathrm{c} i}(x(t)) u_{\mathrm{c}}(t)\right]^{\mathrm{T}}\left[\ell_{\mathrm{c} i}(x(t))+\mathscr{E}_{\mathrm{c} i}(x(t)) u_{\mathrm{c}}(t)\right], \\
& t_{k}<t \leq t_{k+1}, i=1, \ldots, q, \\
& v_{\mathrm{s} i}\left(x\left(t_{k}\right)+\mathscr{F}_{\mathrm{d}}\left(x\left(t_{k}\right)\right)+G_{\mathrm{d}}\left(x\left(t_{k}\right)\right) u_{\mathrm{d}}\left(t_{k}\right)\right)-v_{\mathrm{si}}\left(x\left(t_{k}\right)\right) \\
&=s_{\mathrm{d} i}\left(u_{\mathrm{d}}\left(t_{k}\right), y_{\mathrm{d}}\left(t_{k}\right)\right)-\left[\ell_{\mathrm{d} i}\left(x\left(t_{k}\right)\right)+\mathscr{L}_{\mathrm{d} i}\left(x\left(t_{k}\right)\right) u_{\mathrm{d}}\left(t_{k}\right)\right]^{\mathrm{T}} \\
& \times\left[\ell_{\mathrm{d} i}\left(x\left(t_{k}\right)\right)+\mathscr{L}_{\mathrm{d} i}\left(x\left(t_{k}\right)\right) u_{\mathrm{d}}\left(t_{k}\right)\right], \quad k \in \overline{\mathbb{Z}}_{+},
\end{aligned}
$$

which yields a set of $q$ generalized energy conservation equations for the large-scale impulsive dynamical system $\mathscr{G}$. Specifically, (4.13) shows that the rate of change in generalized energy, or generalized power, over the time interval $t \in\left(t_{k}, t_{k+1}\right]$ for the $i$ th subsystem of $\mathscr{G}$ is equal to the generalized system power input to the $i$ th subsystem plus the instantaneous rate of energy supplied to the ith subsystem from the net energy flow between all subsystems minus the internal generalized system power dissipated from the $i$ th subsystem, while (4.14) shows that the change of energy at the resetting times $t_{k}, k \in \overline{\mathbb{Z}}_{+}$, is 
equal to the external generalized system supplied energy at the resetting times minus the generalized dissipated energy at the resetting times.

Remark 4.5. Note that if $\mathscr{G}$ with $\left(u_{\mathrm{c}}(t), u_{\mathrm{d}}\left(t_{k}\right)\right) \equiv(0,0)$ is vector dissipative (resp., exponentially vector dissipative) with respect to the quadratic hybrid supply rate, where $Q_{\mathrm{c} i} \leq 0, Q_{\mathrm{d} i} \leq 0, i=1, \ldots, q$, then it follows from the vector hybrid dissipation inequality that for all $k \in \overline{\mathbb{Z}}_{+}$,

$$
\begin{gathered}
\dot{V}_{\mathrm{s}}(x(t)) \leq \leq w_{\mathrm{c}}\left(V_{\mathrm{s}}(x(t))\right)+S_{\mathrm{c}}\left(0, y_{\mathrm{c}}(t)\right) \leq \leq w_{\mathrm{c}}\left(V_{\mathrm{s}}(x(t))\right), \quad t_{k}<t \leq t_{k+1}, \\
V_{\mathrm{s}}\left(x\left(t_{k}\right)+\mathscr{F}_{\mathrm{d}}\left(x\left(t_{k}\right)\right)\right)-V_{\mathrm{s}}\left(x\left(t_{k}\right)\right) \leq \leq S_{\mathrm{d}}\left(0, y_{\mathrm{d}}\left(t_{k}\right)\right) \leq \leq 0
\end{gathered}
$$

where $S_{\mathrm{c}}\left(0, y_{\mathrm{c}}\right)=\left[s_{\mathrm{c} 1}\left(0, y_{\mathrm{c} 1}\right), \ldots, s_{\mathrm{c} q}\left(0, y_{\mathrm{c} q}\right)\right]^{\mathrm{T}}, \quad S_{\mathrm{d}}\left(0, y_{\mathrm{d}}\right)=\left[s_{\mathrm{d} 1}\left(0, y_{\mathrm{d} 1}\right), \ldots, s_{\mathrm{d} q}\left(0, y_{\mathrm{d} q}\right)\right]^{\mathrm{T}}$, $s_{\mathrm{c} i}\left(0, y_{\mathrm{c} i}(t)\right)=y_{\mathrm{c} i}^{\mathrm{T}}(t) Q_{\mathrm{c} i} y_{\mathrm{c} i}(t) \leq 0, s_{\mathrm{d} i}\left(0, y_{\mathrm{d} i}\left(t_{k}\right)\right)=y_{\mathrm{d} i}^{\mathrm{T}}\left(t_{k}\right) Q_{\mathrm{d} i} y_{\mathrm{d} i}\left(t_{k}\right) \leq 0, t_{k}<t \leq t_{k+1}, k \in$ $\overline{\mathbb{Z}}_{+}, i=1, \ldots, q$, and $x(t), t \geq t_{0}$, is the solution to (3.1), (3.2), (3.3), and (3.4) with $\left(u_{\mathrm{c}}(t)\right.$, $\left.u_{\mathrm{d}}\left(t_{k}\right)\right) \equiv(0,0)$. If, in addition, there exists $p \in \mathbb{R}_{+}^{q}$ such that $p^{\mathrm{T}} V_{\mathrm{s}}(x), x \in \mathbb{R}^{n}$, is positivedefinite, then it follows from Theorem 2.7 that the undisturbed $\left(\left(u_{\mathrm{c}}(t), u_{\mathrm{d}}\left(t_{k}\right)\right) \equiv(0,0)\right)$ large-scale impulsive dynamical system (3.1), (3.2), (3.3), and (3.4) is Lyapunov (resp., asymptotically) stable.

Next, we consider a specialization of Theorem 4.4 wherein $\mathscr{G}$ is a linear impulsive dynamical system. Specifically, we assume that $w_{\mathrm{c}} \in \mathcal{W}$ is linear so that $w_{\mathrm{c}}(r)=W r$, where $W \in \mathbb{R}^{q \times q}$ is essentially nonnegative, and consider the large-scale linear impulsive dynamical system $\mathscr{G}$ given by

$$
\begin{aligned}
\dot{x}(t) & =A_{\mathrm{c}} x(t)+B_{\mathrm{c}} u_{\mathrm{c}}(t), \quad x(t) \notin \mathscr{E}_{x}, \\
\Delta x(t) & =\left(A_{\mathrm{d}}-I_{n}\right) x(t)+B_{\mathrm{d}} u_{\mathrm{d}}(t), \quad x(t) \in \mathscr{E}_{x}, \\
y_{\mathrm{c}}(t) & =C_{\mathrm{c}} x(t)+D_{\mathrm{c}} u_{\mathrm{c}}(t), \quad x(t) \notin \mathscr{E}_{x}, \\
y_{\mathrm{d}}(t) & =C_{\mathrm{d}} x(t)+D_{\mathrm{d}} u_{\mathrm{d}}(t), \quad x(t) \in \mathscr{E}_{x},
\end{aligned}
$$

where $A_{\mathrm{c}} \in \mathbb{R}^{n \times n}$ and is partitioned as $A_{\mathrm{c}} \triangleq\left[A_{\mathrm{c} i j}\right], i, j=1, \ldots, q, A_{\mathrm{c} i j} \in \mathbb{R}^{n_{i} \times n_{j}}, \sum_{i=1}^{q} n_{i}$ $=n, B_{\mathrm{c}}=$ block-diag $\left[B_{\mathrm{c} 1}, \ldots, B_{\mathrm{c} q}\right], C_{\mathrm{c}}=\operatorname{block}-\operatorname{diag}\left[C_{\mathrm{c} 1}, \ldots, C_{\mathrm{c} q}\right], D_{\mathrm{c}}=\operatorname{block}-\operatorname{diag}\left[D_{\mathrm{c} 1}\right.$, $\left.\ldots, D_{\mathrm{c} q}\right], B_{\mathrm{c} i} \in \mathbb{R}^{n_{i} \times m_{\mathrm{c} i}}, C_{\mathrm{c} i} \in \mathbb{R}^{l_{\mathrm{c} i} \times n_{i}}, D_{\mathrm{c} i} \in \mathbb{R}^{l_{\mathrm{c} i} \times m_{\mathrm{c} i}} ;$ and $A_{\mathrm{d}} \in \mathbb{R}^{n \times n}$ and is partitioned as $A_{\mathrm{d}} \triangleq\left[A_{\mathrm{d} i j}\right], \quad i, j=1, \ldots, q, \quad A_{\mathrm{d} i j} \in \mathbb{R}^{n_{i} \times n_{j}}, \quad B_{\mathrm{d}}=\operatorname{block}-\operatorname{diag}\left[B_{\mathrm{d} 1}, \ldots, B_{\mathrm{d} q}\right], \quad C_{\mathrm{d}}=$ block-diag $\left[C_{\mathrm{d} 1}, \ldots, C_{\mathrm{d} q}\right], D_{\mathrm{d}}=\operatorname{block}-\operatorname{diag}\left[D_{\mathrm{d} 1}, \ldots, D_{\mathrm{d} q}\right], B_{\mathrm{d} i} \in \mathbb{R}^{n_{i} \times m_{\mathrm{d} i}}, C_{\mathrm{d} i} \in \mathbb{R}^{l_{\mathrm{di}} \times n_{i}}, D_{\mathrm{d} i} \in$ $\mathbb{R}^{l_{\mathrm{d} i} \times m_{\mathrm{d} i}}$, and $i=1, \ldots, q$.

Corollary 4.6. Consider the large-scale linear impulsive dynamical system $\mathscr{G}_{\text {given }}$ by (4.16). Let $R_{\mathrm{c} i} \in \mathbb{S}^{m_{\mathrm{c} i}}, S_{\mathrm{c} i} \in \mathbb{R}^{l_{\mathrm{c} i} \times m_{\mathrm{c} i}}, Q_{\mathrm{c} i} \in \mathbb{S}^{l_{\mathrm{c} i}}, R_{\mathrm{d} i} \in \mathbb{S}^{m_{\mathrm{d} i}}, S_{\mathrm{d} i} \in \mathbb{R}^{l_{\mathrm{d} i} \times m_{\mathrm{d} i}}$, and $Q_{\mathrm{d} i} \in \mathbb{S}^{l_{\mathrm{d} i}}$, $i=1, \ldots, q$. Then $\mathscr{G}$ is vector dissipative (resp., exponentially vector dissipative) with respect to the vector hybrid supply rate $\left(S_{\mathrm{c}}\left(u_{\mathrm{c}}, y_{\mathrm{c}}\right), S_{\mathrm{d}}\left(u_{\mathrm{d}}, y_{\mathrm{d}}\right)\right)$, where $s_{\mathrm{c} i}\left(u_{\mathrm{c} i}, y_{\mathrm{c} i}\right)=u_{\mathrm{c} i}^{\mathrm{T}} R_{\mathrm{c} i} u_{\mathrm{c} i}+$ $2 y_{\mathrm{c} i}^{\mathrm{T}} S_{\mathrm{c} i} u_{\mathrm{c} i}+y_{\mathrm{c} i}^{\mathrm{T}} Q_{\mathrm{c} i} y_{\mathrm{c} i}$ and $s_{\mathrm{d} i}\left(u_{\mathrm{d} i}, y_{\mathrm{d} i}\right)=u_{\mathrm{d} i}^{\mathrm{T}} R_{\mathrm{d} i} u_{\mathrm{d} i}+2 y_{\mathrm{d} i}^{\mathrm{T}} S_{\mathrm{d} i} u_{\mathrm{d} i}+y_{\mathrm{d} i}^{\mathrm{T}} Q_{\mathrm{d} i} y_{\mathrm{d} i}, i=1, \ldots, q$, if there exist $W \in \mathbb{R}^{q \times q}, P_{i} \in \mathbb{N}^{n}, L_{\mathrm{c} i} \in \mathbb{R}^{s_{\mathrm{c} i} \times n}, Z_{\mathrm{c} i} \in \mathbb{R}^{s_{\mathrm{c} i} \times m_{\mathrm{c}}}, L_{\mathrm{d} i} \in \mathbb{R}^{s_{\mathrm{d} i} \times n}$, and $Z_{\mathrm{d} i} \in \mathbb{R}^{s_{\mathrm{d} i} \times m_{\mathrm{d}}}$, $i=1, \ldots, q$, such that $W$ is essentially nonnegative and semistable (resp., asymptotically 
stable), and, for all $i=1, \ldots, q$,

$$
\begin{aligned}
& 0=x^{\mathrm{T}}\left(A_{\mathrm{c}}^{\mathrm{T}} P_{i}+P_{i} A_{\mathrm{c}}-C_{\mathrm{c}}^{\mathrm{T}} \hat{Q}_{\mathrm{c} i} C_{\mathrm{c}}-\sum_{j=1}^{q} W_{(i, j)} P_{j}+L_{\mathrm{c} i}^{\mathrm{T}} L_{\mathrm{c} i}\right) x, \quad x \notin \mathscr{E}_{x}, \\
& 0=x^{\mathrm{T}}\left(P_{i} B_{\mathrm{c}}-C_{\mathrm{c}}^{\mathrm{T}}\left(\hat{S}_{\mathrm{c} i}+\hat{Q}_{\mathrm{c} i} D_{\mathrm{c}}\right)+L_{\mathrm{c} i}^{\mathrm{T}} Z_{\mathrm{c} i}\right), \quad x \notin \mathscr{L}_{x}, \\
& 0=\hat{R}_{\mathrm{c} i}+D_{\mathrm{c}}^{\mathrm{T}} \hat{S}_{\mathrm{c} i}+\hat{S}_{\mathrm{c} i}^{\mathrm{T}} D_{\mathrm{c}}+D_{\mathrm{c}}^{\mathrm{T}} \hat{Q}_{\mathrm{c} i} D_{\mathrm{c}}-Z_{\mathrm{c} i}^{\mathrm{T}} Z_{\mathrm{c} i}, \\
& 0=x^{\mathrm{T}}\left(A_{\mathrm{d}}^{\mathrm{T}} P_{i} A_{\mathrm{d}}-C_{\mathrm{d}}^{\mathrm{T}} \hat{Q}_{\mathrm{d} i} C_{\mathrm{d}}-P_{i}+L_{\mathrm{d} i}^{\mathrm{T}} L_{\mathrm{d} i}\right) x, \quad x \in \mathscr{Z}_{x}, \\
& 0=x^{\mathrm{T}}\left(A_{\mathrm{d}}^{\mathrm{T}} P_{i} B_{\mathrm{d}}-C_{\mathrm{d}}^{\mathrm{T}}\left(\hat{S}_{\mathrm{d} i}+\hat{Q}_{\mathrm{d} i} D_{\mathrm{d}}\right)+L_{\mathrm{d} i}^{\mathrm{T}} Z_{\mathrm{d} i}\right), \quad x \in \mathscr{Z}_{x}, \\
& 0=\hat{R}_{\mathrm{d} i}+D_{\mathrm{d}}^{\mathrm{T}} \hat{S}_{\mathrm{d} i}+\hat{S}_{\mathrm{d} i}^{\mathrm{T}} D_{\mathrm{d}}+D_{\mathrm{d}}^{\mathrm{T}} \hat{Q}_{\mathrm{d} i} D_{\mathrm{d}}-B_{\mathrm{d}}^{\mathrm{T}} P_{i} B_{\mathrm{d}}-Z_{\mathrm{d} i}^{\mathrm{T}} Z_{\mathrm{d} i} .
\end{aligned}
$$

Proof. The proof follows from Theorem 4.4 with $\mathscr{F}_{\mathrm{c}}(x)=A_{\mathrm{c}} x, G_{\mathrm{c}}(x)=B_{\mathrm{c}}, h_{\mathrm{c}}(x)=C_{\mathrm{c}} x$, $J_{\mathrm{c}}(x)=D_{\mathrm{c}}, w_{\mathrm{c}}(r)=W r, \ell_{\mathrm{c} i}(x)=L_{\mathrm{c} i} x, \mathscr{L}_{\mathrm{c} i}(x)=Z_{\mathrm{c} i}, \mathscr{F}_{\mathrm{d}}(x)=\left(A_{\mathrm{d}}-I\right) x, G_{\mathrm{d}}(x)=B_{\mathrm{d}}$, $h_{\mathrm{d}}(x)=C_{\mathrm{d}} x, J_{\mathrm{d}}(x)=D_{\mathrm{d}}, \ell_{\mathrm{d} i}(x)=L_{\mathrm{d} i} x, \mathscr{L}_{\mathrm{d} i}(x)=Z_{\mathrm{d} i}, P_{1 i}(x)=2 x^{\mathrm{T}} A_{\mathrm{d}}^{\mathrm{T}} P_{i} B_{\mathrm{d}}, P_{2 i}(x)=$ $B_{\mathrm{d}}^{\mathrm{T}} P_{i} B_{\mathrm{d}}$, and $v_{\mathrm{si}}(x)=x^{\mathrm{T}} P_{i} x, i=1, \ldots, q$.

Remark 4.7. Note that (4.17), (4.18), (4.19), (4.20), (4.21), and (4.22) are implied by

$$
\begin{aligned}
& {\left[\begin{array}{ll}
\mathscr{A}_{\mathrm{c} i} & \mathscr{B}_{\mathrm{c} i} \\
\mathscr{B}_{\mathrm{c} i}^{\mathrm{T}} & \mathscr{C}_{\mathrm{c} i}
\end{array}\right]=-\left[\begin{array}{l}
L_{\mathrm{c} i}^{\mathrm{T}} \\
Z_{\mathrm{c} i}^{\mathrm{T}}
\end{array}\right]\left[\begin{array}{ll}
L_{\mathrm{c} i} & Z_{\mathrm{c} i}
\end{array}\right] \leq 0,} \\
& {\left[\begin{array}{ll}
\mathscr{A}_{\mathrm{d} i} & \mathscr{O}_{\mathrm{d} i} \\
\mathscr{B}_{\mathrm{d} i}^{\mathrm{T}} & \mathscr{C}_{\mathrm{d} i}
\end{array}\right]=-\left[\begin{array}{l}
L_{\mathrm{d} i}^{\mathrm{T}} \\
Z_{\mathrm{d} i}^{\mathrm{T}}
\end{array}\right]\left[\begin{array}{ll}
L_{\mathrm{d} i} & Z_{\mathrm{d} i}
\end{array}\right] \leq 0, \quad i=1, \ldots, q,}
\end{aligned}
$$

where, for all $i=1, \ldots, q$,

$$
\begin{aligned}
& \mathscr{A}_{\mathrm{c} i}=A_{\mathrm{c}}^{\mathrm{T}} P_{i}+P_{i} A_{\mathrm{c}}-C_{\mathrm{c}}^{\mathrm{T}} \hat{Q}_{\mathrm{c} i} C_{\mathrm{c}}-\sum_{j=1}^{q} W_{(i, j)} P_{j}, \\
& \mathscr{B}_{\mathrm{c} i}=P_{i} B_{\mathrm{c}}-C_{\mathrm{c}}^{\mathrm{T}}\left(\hat{S}_{\mathrm{c} i}+\hat{Q}_{\mathrm{c} i} D_{\mathrm{c}}\right), \\
& \mathscr{C}_{\mathrm{c} i}=-\left(\hat{R}_{\mathrm{c} i}+D_{\mathrm{c}}^{\mathrm{T}} \hat{S}_{\mathrm{c} i}+\hat{S}_{\mathrm{c} i}^{\mathrm{T}} D_{\mathrm{c}}+D_{\mathrm{c}}^{\mathrm{T}} \hat{Q}_{\mathrm{c} i} D_{\mathrm{c}}\right), \\
& \mathscr{A}_{\mathrm{d} i}=A_{\mathrm{d}}^{\mathrm{T}} P_{i} A_{\mathrm{d}}-C_{\mathrm{d}}^{\mathrm{T}} \hat{Q}_{\mathrm{d} i} C_{\mathrm{d}}-P_{i}, \\
& \mathscr{B}_{\mathrm{d} i}=A_{\mathrm{d}}^{\mathrm{T}} P_{i} B_{\mathrm{d}}-C_{\mathrm{d}}^{\mathrm{T}}\left(\hat{S}_{\mathrm{d} i}+\hat{Q}_{\mathrm{d} i} D_{\mathrm{d}}\right), \\
& \mathscr{C}_{\mathrm{d} i}=-\left(\hat{R}_{\mathrm{d} i}+D_{\mathrm{d}}^{\mathrm{T}} \hat{S}_{\mathrm{d} i}+\hat{S}_{\mathrm{d} i}^{\mathrm{T}} D_{\mathrm{d}}+D_{\mathrm{d}}^{\mathrm{T}} \hat{Q}_{\mathrm{d} i} D_{\mathrm{d}}-B_{\mathrm{d}}^{\mathrm{T}} P_{i} B_{\mathrm{d}}\right) .
\end{aligned}
$$

Hence, vector dissipativity of large-scale linear impulsive dynamical systems with respect to quadratic hybrid supply rates can be characterized via (cascade) linear matrix inequalities (LMIs) [6].

Next, we extend the notions of passivity and nonexpansivity to vector passivity and vector nonexpansivity. 
Definition 4.8. The large-scale impulsive dynamical system $G$ given by (3.1), (3.2), (3.3), and (3.4) with $m_{\mathrm{c} i}=l_{\mathrm{c} i}, m_{\mathrm{d} i}=l_{\mathrm{d} i}, i=1, \ldots, q$, is vector passive (resp., vector exponentially passive) if it is vector dissipative (resp., exponentially vector dissipative) with respect to the vector hybrid supply rate $\left(S_{\mathrm{c}}\left(u_{\mathrm{c}}, y_{\mathrm{c}}\right), S_{\mathrm{d}}\left(u_{\mathrm{d}}, y_{\mathrm{d}}\right)\right)$, where $s_{\mathrm{c} i}\left(u_{\mathrm{c} i}, y_{\mathrm{c} i}\right)=2 y_{\mathrm{c} i}^{\mathrm{T}} u_{\mathrm{c} i}$ and $s_{\mathrm{d} i}\left(u_{\mathrm{d} i}, y_{\mathrm{d} i}\right)=2 y_{\mathrm{d} i}^{\mathrm{T}} u_{\mathrm{d} i}, i=1, \ldots, q$.

Definition 4.9. The large-scale impulsive dynamical system $\mathscr{G}$ given by (3.1), (3.2), (3.3), and (3.4) is vector nonexpansive (resp., vector exponentially nonexpansive) if it is vector dissipative (resp., exponentially vector dissipative) with respect to the vector hybrid supply rate $\left(S_{\mathrm{c}}\left(u_{\mathrm{c}}, y_{\mathrm{c}}\right), S_{\mathrm{d}}\left(u_{\mathrm{d}}, y_{\mathrm{d}}\right)\right)$, where $s_{\mathrm{c} i}\left(u_{\mathrm{c} i}, y_{\mathrm{c} i}\right)=\gamma_{\mathrm{c} i}^{2} u_{\mathrm{c} i}^{\mathrm{T}} u_{\mathrm{c} i}-y_{\mathrm{c} i}^{\mathrm{T}} y_{\mathrm{c} i}$ and $s_{\mathrm{d} i}\left(u_{\mathrm{d} i}, y_{\mathrm{d} i}\right)=$ $\gamma_{\mathrm{d} i}^{2} u_{\mathrm{d} i}^{\mathrm{T}} u_{\mathrm{d} i}-y_{\mathrm{d} i}^{\mathrm{T}} y_{\mathrm{d} i}, i=1, \ldots, q$, and $\gamma_{\mathrm{c} i}>0, \gamma_{\mathrm{d} i}>0, i=1, \ldots, q$, are given.

Remark 4.10. Note that a mixed vector passive-nonexpansive formulation of $\mathscr{G}$ can also be considered. Specifically, one can consider large-scale impulsive dynamical systems $\mathscr{G}$ which are vector dissipative with respect to hybrid vector supply rates $\left(S_{\mathrm{c}}\left(u_{\mathrm{c}}, y_{\mathrm{c}}\right), S_{\mathrm{d}}\left(u_{\mathrm{d}}\right.\right.$, $\left.\left.y_{\mathrm{d}}\right)\right)$, where $s_{\mathrm{c} i}\left(u_{\mathrm{c} i}, y_{\mathrm{c} i}\right)=2 y_{\mathrm{c} i}^{\mathrm{T}} u_{\mathrm{c} i}, s_{\mathrm{d} i}\left(u_{\mathrm{d} i}, y_{\mathrm{d} i}\right)=2 y_{\mathrm{d} i}^{\mathrm{T}} u_{\mathrm{d} i}, i \in \mathbb{Z}_{\mathrm{p}}, s_{\mathrm{c} j}\left(u_{\mathrm{c} j}, y_{\mathrm{c} j}\right)=\gamma_{\mathrm{c} j}^{2} u_{\mathrm{c} j}^{\mathrm{T}} u_{\mathrm{c} j}-$ $y_{\mathrm{c} j}^{\mathrm{T}} y_{\mathrm{c} j}, \gamma_{\mathrm{c} j}>0, s_{\mathrm{d} j}\left(u_{\mathrm{d} j}, y_{\mathrm{d} j}\right)=\gamma_{\mathrm{d} j}^{2} u_{\mathrm{d} j}^{\mathrm{T}} u_{\mathrm{d} j}-y_{\mathrm{d} j}^{\mathrm{T}} y_{\mathrm{d} j}, \gamma_{\mathrm{d} j}>0, j \in \mathbb{Z}_{\mathrm{ne}}, \mathbb{Z}_{\mathrm{p}} \cap \mathbb{Z}_{\mathrm{ne}}=\varnothing$, and $\mathbb{Z}_{\mathrm{p}} \cup$ $\mathbb{Z}_{\mathrm{ne}}=\{1, \ldots, q\}$. Furthermore, hybrid supply rates for vector input strict passivity, vector output strict passivity, and vector input-output strict passivity generalizing the passivity notions given in [14] can also be considered. However, for simplicity of exposition, we do not do so here.

The next result presents constructive sufficient conditions guaranteeing vector dissipativity of $\mathscr{G}$ with respect to a quadratic hybrid supply rate for the case where the vector storage function $V_{s}(x), x \in \mathbb{R}^{n}$, is component decoupled; that is, $V_{s}(x)=\left[v_{s 1}\left(x_{1}\right), \ldots\right.$, $\left.v_{\mathrm{s} q}\left(x_{q}\right)\right]^{\mathrm{T}}, x \in \mathbb{R}^{n}$.

Theorem 4.11. Consider the large-scale impulsive dynamical system $G_{\text {given }}$ by (3.1), (3.2), (3.3), and (3.4). Assume that there exist functions $V_{\mathrm{s}}=\left[v_{\mathrm{s} 1}, \ldots, v_{\mathrm{sq}}\right]^{\mathrm{T}}: \mathbb{R}^{n} \rightarrow \overline{\mathbb{R}}_{+}^{q}$, $w_{\mathrm{c}}=\left[w_{\mathrm{cl}}, \ldots, w_{\mathrm{c} q}\right]^{\mathrm{T}}: \overline{\mathbb{R}}_{+}^{q} \rightarrow \mathbb{R}^{q}, \ell_{\mathrm{c} i}: \mathbb{R}^{n} \rightarrow \mathbb{R}^{s_{\mathrm{c} i}}, \mathscr{E}_{\mathrm{c} i}: \mathbb{R}^{n} \rightarrow \mathbb{R}^{s_{\mathrm{c} i} \times m_{\mathrm{c} i}}, \ell_{\mathrm{d} i}: \mathbb{R}^{n} \rightarrow \mathbb{R}^{s_{\mathrm{d} i}}, \mathscr{E}_{\mathrm{d} i}:$ $\mathbb{R}^{n} \rightarrow \mathbb{R}^{s_{\mathrm{d} i} \times m_{\mathrm{d} i}}, P_{1 i}: \mathbb{R}^{n} \rightarrow \mathbb{R}^{1 \times m_{\mathrm{d} i}}, P_{2 i}: \mathbb{R}^{n} \rightarrow \mathbb{N}^{m_{\mathrm{d} i}}$ such that $v_{\mathrm{s} i}(\cdot)$ is continuously differentiable, $v_{\mathrm{si}}(0)=0, i=1, \ldots, q, w_{\mathrm{c}} \in \mathcal{W}, w_{\mathrm{c}}(0)=0$, the zero solution $r(t) \equiv 0$ to (2.6) is Lyapunov (resp., asymptotically) stable, and, for all $x \in \mathbb{R}^{n}$ and $i=1, \ldots, q$,

$$
\begin{aligned}
0 \leq & v_{\mathrm{s} i}\left(x_{i}+\mathscr{F}_{\mathrm{d} i}(x)\right)-v_{\mathrm{s} i}\left(x_{i}+\mathscr{F}_{\mathrm{d} i}(x)+G_{\mathrm{d} i}\left(x_{i}\right) u_{\mathrm{d} i}\right) \\
& +P_{1 i}(x) u_{\mathrm{d} i}+u_{\mathrm{d} i}^{\mathrm{T}} P_{2 i}(x) u_{\mathrm{d} i}, \quad x \in \mathscr{L}_{x}, u_{\mathrm{d} i} \in \mathbb{R}^{m_{\mathrm{d} i}}, \\
0 \geq & v_{\mathrm{s} i}^{\prime}\left(x_{i}\right) \mathscr{F}_{\mathrm{c} i}(x)-h_{\mathrm{c} i}^{\mathrm{T}}\left(x_{i}\right) Q_{\mathrm{c} i} h_{\mathrm{c} i}\left(x_{i}\right)-w_{\mathrm{c} i}\left(V_{\mathrm{s}}(x)\right)+\ell_{\mathrm{c} i}^{\mathrm{T}}\left(x_{i}\right) \ell_{\mathrm{c} i}\left(x_{i}\right), \quad x \notin \mathscr{E}_{x}, \\
0= & \frac{1}{2} v_{\mathrm{s} i}^{\prime}\left(x_{i}\right) G_{\mathrm{c} i}\left(x_{i}\right)-h_{\mathrm{c} i}^{\mathrm{T}}\left(x_{i}\right)\left(S_{\mathrm{c} i}+Q_{\mathrm{c} i} J_{\mathrm{c} i}\left(x_{i}\right)\right)+\ell_{\mathrm{c} i}^{\mathrm{T}}\left(x_{i}\right) \mathscr{L}_{\mathrm{c} i}\left(x_{i}\right), \quad x \notin \mathscr{E}_{x}, \\
0 \leq & R_{\mathrm{c} i}+J_{\mathrm{c} i}^{\mathrm{T}}\left(x_{i}\right) S_{\mathrm{c} i}+S_{\mathrm{c} i}^{\mathrm{T}} J_{\mathrm{c} i}\left(x_{i}\right)+J_{\mathrm{c} i}^{\mathrm{T}}\left(x_{i}\right) Q_{\mathrm{c} i} J_{\mathrm{c} i}\left(x_{i}\right)-\mathscr{L}_{\mathrm{c} i}^{\mathrm{T}}\left(x_{i}\right) \mathscr{L}_{\mathrm{c} i}\left(x_{i}\right), \quad x \notin \mathscr{L}_{x}, \\
0 \geq & v_{\mathrm{s} i}\left(x_{i}+\mathscr{F}_{\mathrm{d} i}(x)\right)-h_{\mathrm{d} i}^{\mathrm{T}}\left(x_{i}\right) Q_{\mathrm{d} i} h_{\mathrm{d} i}\left(x_{i}\right)-v_{\mathrm{s} i}\left(x_{i}\right)+\ell_{\mathrm{d} i}^{\mathrm{T}}\left(x_{i}\right) \ell_{\mathrm{d} i}\left(x_{i}\right), \quad x \in \mathscr{L}_{x}, \\
0= & \frac{1}{2} P_{1 i}(x)-h_{\mathrm{d} i}^{\mathrm{T}}\left(x_{i}\right)\left(S_{\mathrm{d} i}+Q_{\mathrm{d} i} J_{\mathrm{d} i}\left(x_{i}\right)\right)+\ell_{\mathrm{d} i}^{\mathrm{T}}\left(x_{i}\right) \mathscr{L}_{\mathrm{d} i}\left(x_{i}\right), \quad x \in \mathscr{E}_{x},
\end{aligned}
$$




$$
\begin{aligned}
0 \leq & R_{\mathrm{d} i}+J_{\mathrm{d} i}^{\mathrm{T}}\left(x_{i}\right) S_{\mathrm{d} i}+S_{\mathrm{d} i}^{\mathrm{T}} J_{\mathrm{d} i}\left(x_{i}\right)+J_{\mathrm{d} i}^{\mathrm{T}}\left(x_{i}\right) Q_{\mathrm{d} i} J_{\mathrm{d} i}\left(x_{i}\right) \\
& -P_{2 i}(x)-\mathscr{L}_{\mathrm{d} i}^{\mathrm{T}}\left(x_{i}\right) \mathscr{L}_{\mathrm{d} i}\left(x_{i}\right), \quad x \in \mathscr{L}_{x} .
\end{aligned}
$$

Then $G$ is vector dissipative (resp., exponentially vector dissipative) with respect to the vector hybrid supply rate $\left(S_{\mathrm{c}}\left(u_{\mathrm{c}}, y_{\mathrm{c}}\right), S_{\mathrm{d}}\left(u_{\mathrm{d}}, y_{\mathrm{d}}\right)\right)$, where $s_{\mathrm{c} i}\left(u_{\mathrm{c} i}, y_{\mathrm{c} i}\right)=u_{\mathrm{c} i}^{\mathrm{T}} R_{\mathrm{c} i} u_{\mathrm{c} i}+2 y_{\mathrm{c} i}^{\mathrm{T}} S_{\mathrm{c} i} u_{\mathrm{c} i}+$ $y_{\mathrm{c} i}^{\mathrm{T}} Q_{\mathrm{c} i} y_{\mathrm{c} i}$ and $s_{\mathrm{d} i}\left(u_{\mathrm{d} i}, y_{\mathrm{d} i}\right)=u_{\mathrm{d} i}^{\mathrm{T}} R_{\mathrm{d} i} u_{\mathrm{d} i}+2 y_{\mathrm{d} i}^{\mathrm{T}} S_{\mathrm{d} i} u_{\mathrm{d} i}+y_{\mathrm{d} i}^{\mathrm{T}} Q_{\mathrm{d} i} y_{\mathrm{d} i}, i=1, \ldots, q$.

Proof. The proof is similar to the proof of Theorem 4.4 and hence is omitted.

Finally, we provide necessary and sufficient conditions for the case where the largescale impulsive dynamical system $\mathscr{G}$ is vector lossless with respect to a quadratic hybrid supply rate.

Theorem 4.12. Consider the large-scale impulsive dynamical system $\mathcal{G}$ given by (3.1), (3.2), (3.3), and (3.4). Let $R_{\mathrm{c} i} \in \mathbb{S}^{m_{\mathrm{c} i}}, S_{\mathrm{c} i} \in \mathbb{R}^{l_{\mathrm{c} i} \times m_{\mathrm{c} i}}, Q_{\mathrm{c} i} \in \mathbb{S}^{l_{c i}}, R_{\mathrm{d} i} \in \mathbb{S}^{m_{\mathrm{d} i}}, S_{\mathrm{d} i} \in \mathbb{R}^{l_{\mathrm{d} i} \times m_{\mathrm{d} i}}$, and $Q_{\mathrm{d} i} \in \mathbb{S}^{l_{\mathrm{d} i}}, i=1, \ldots, q$. Then $\varphi_{\mathrm{G}}$ is vector lossless with respect to the quadratic hybrid supply rate $\left(S_{\mathrm{c}}\left(u_{\mathrm{c}}, y_{\mathrm{c}}\right), S_{\mathrm{d}}\left(u_{\mathrm{d}}, y_{\mathrm{d}}\right)\right)$, where $s_{\mathrm{c} i}\left(u_{\mathrm{c} i}, y_{\mathrm{c} i}\right)=u_{\mathrm{c} i}^{\mathrm{T}} R_{\mathrm{c} i} u_{\mathrm{c} i}+2 y_{\mathrm{c} i}^{\mathrm{T}} S_{\mathrm{c} i} u_{\mathrm{c} i}+y_{\mathrm{c} i}^{\mathrm{T}} Q_{\mathrm{c} i} y_{\mathrm{c} i}$ and $s_{\mathrm{d} i}\left(u_{\mathrm{d} i}, y_{\mathrm{d} i}\right)=u_{\mathrm{d} i}^{\mathrm{T}} R_{\mathrm{d} i} u_{\mathrm{d} i}+2 y_{\mathrm{d} i}^{\mathrm{T}} S_{\mathrm{d} i} u_{\mathrm{d} i}+y_{\mathrm{d} i}^{\mathrm{T}} Q_{\mathrm{d} i} y_{\mathrm{d} i}, i=1, \ldots, q$, if and only if there exist functions $V_{\mathrm{s}}=\left[v_{\mathrm{s} 1}, \ldots, v_{\mathrm{s} q}\right]^{\mathrm{T}}: \mathbb{R}^{n} \rightarrow \overline{\mathbb{R}}_{+}^{q}, P_{1 i}: \mathbb{R}^{n} \rightarrow \mathbb{R}^{1 \times m_{\mathrm{d}}}, P_{2 i}: \mathbb{R}^{n} \rightarrow \mathbb{N}^{m_{\mathrm{d}}}$, and $w_{\mathrm{c}}=\left[w_{\mathrm{c} 1}, \ldots\right.$, $\left.w_{\mathrm{c} q}\right]^{\mathrm{T}}: \overline{\mathbb{R}}_{+}^{q} \rightarrow \mathbb{R}^{q}$ such that $v_{\mathrm{si}}(\cdot)$ is continuously differentiable, $v_{\mathrm{s} i}(0)=0, i=1, \ldots, q, w_{\mathrm{c}} \in$ $\mathcal{W}, w_{\mathrm{c}}(0)=0$, the zero solution $r(t) \equiv 0$ to (2.6) is Lyapunov stable, and, for all $x \in \mathbb{R}^{n}$, $i=1, \ldots, q,(4.3)$ holds and

$$
\begin{aligned}
0 & =v_{\mathrm{s} i}^{\prime}(x) \mathscr{F}_{\mathrm{c}}(x)-h_{\mathrm{c}}^{\mathrm{T}}(x) \hat{Q}_{\mathrm{c} i} h_{\mathrm{c}}(x)-w_{\mathrm{c} i}\left(V_{\mathrm{s}}(x)\right), \quad x \notin \mathscr{E}_{x}, \\
0 & =\frac{1}{2} v_{\mathrm{s} i}^{\prime}(x) G_{\mathrm{c}}(x)-h_{\mathrm{c}}^{\mathrm{T}}(x)\left(\hat{S}_{\mathrm{c} i}+\hat{Q}_{\mathrm{c} i} J_{\mathrm{c}}(x)\right), \quad x \notin \mathscr{L}_{x}, \\
0 & =\hat{R}_{\mathrm{c} i}+J_{\mathrm{c}}^{\mathrm{T}}(x) \hat{S}_{\mathrm{c} i}+\hat{S}_{\mathrm{c} i}^{\mathrm{T}} J_{\mathrm{c}}(x)+J_{\mathrm{c}}^{\mathrm{T}}(x) \hat{Q}_{\mathrm{c} i} J_{\mathrm{c}}(x), \quad x \notin \mathscr{E}_{x}, \\
0 & =v_{\mathrm{s} i}\left(x+\mathscr{F}_{\mathrm{d}}(x)\right)-h_{\mathrm{d}}^{\mathrm{T}}(x) \hat{Q}_{\mathrm{d} i} h_{\mathrm{d}}(x)-v_{\mathrm{s} i}(x), \quad x \in \mathscr{E}_{x}, \\
0 & =\frac{1}{2} P_{1 i}(x)-h_{\mathrm{d}}^{\mathrm{T}}(x)\left(\hat{S}_{\mathrm{d} i}+\hat{Q}_{\mathrm{d} i} J_{\mathrm{d}}(x)\right), \quad x \in \mathscr{E}_{x}, \\
0 & =\hat{R}_{\mathrm{d} i}+J_{\mathrm{d}}^{\mathrm{T}}(x) \hat{S}_{\mathrm{d} i}+\hat{S}_{\mathrm{d} i}^{\mathrm{T}} J_{\mathrm{d}}(x)+J_{\mathrm{d}}^{\mathrm{T}}(x) \hat{Q}_{\mathrm{d} i} J_{\mathrm{d}}(x)-P_{2 i}(x), \quad x \in \mathscr{E}_{x} .
\end{aligned}
$$

Proof. Sufficiency follows as in the proof of Theorem 4.4. To show necessity, suppose that

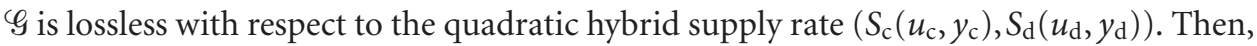
there exist continuous functions $V_{\mathrm{s}}=\left[v_{\mathrm{s} 1}, \ldots, v_{\mathrm{s} q}\right]^{\mathrm{T}}: \mathbb{R}^{n} \rightarrow \overline{\mathbb{R}}_{+}^{q}$ and $w_{\mathrm{c}}=\left[w_{\mathrm{cl}}, \ldots, w_{\mathrm{c} q}\right]^{\mathrm{T}}$ : $\overline{\mathbb{R}}_{+}^{q} \rightarrow \mathbb{R}^{q}$ such that $V_{\mathrm{s}}(0)=0$, the zero solution $r(t) \equiv 0$ to (2.6) is Lyapunov stable, and, for all $k \in \overline{\mathbb{Z}}_{+}, i=1, \ldots, q$,

$$
\begin{gathered}
v_{\mathrm{s} i}(x(\hat{t}))-v_{\mathrm{s} i}(x(t))=\int_{t}^{\hat{t}} s_{\mathrm{c} i}\left(u_{\mathrm{c} i}(\sigma), y_{\mathrm{c} i}(\sigma)\right) \mathrm{d} \sigma \\
+\int_{t}^{\hat{t}} w_{\mathrm{c} i}\left(V_{\mathrm{s}}(x(\sigma))\right) \mathrm{d} \sigma, \quad t_{k}<t \leq \hat{t} \leq t_{k+1}, \\
v_{\mathrm{s} i}\left(x\left(t_{k}\right)+\mathscr{F}_{\mathrm{d}}\left(x\left(t_{k}\right)\right)+G_{\mathrm{d}}\left(x\left(t_{k}\right)\right) u_{\mathrm{d}}\left(t_{k}\right)\right)=v_{\mathrm{s} i}\left(x\left(t_{k}\right)\right)+s_{\mathrm{d} i}\left(u_{\mathrm{d} i}\left(t_{k}\right), y_{\mathrm{d} i}\left(t_{k}\right)\right) .
\end{gathered}
$$


Now, dividing (4.32) by $\hat{t}-t^{+}$and letting $\hat{t} \rightarrow t^{+},(4.32)$ is equivalent to

$$
\begin{aligned}
\dot{v}_{\mathrm{s} i}(x(t)) & =v_{\mathrm{si}}^{\prime}(x(t))\left[\mathscr{F}_{\mathrm{c}}(x(t))+G_{\mathrm{c}}(x(t)) u_{\mathrm{c}}(t)\right] \\
& =s_{\mathrm{c} i}\left(u_{\mathrm{c} i}(t), y_{\mathrm{c} i}(t)\right)+w_{\mathrm{c} i}\left(V_{\mathrm{s}}(x(t))\right), \quad t_{k}<t \leq t_{k+1} .
\end{aligned}
$$

Next, with $t=t_{0}$, it follows from (4.34) that

$$
\begin{aligned}
v_{\mathrm{s} i}^{\prime}\left(x_{0}\right) & {\left[\mathscr{F}_{\mathrm{c}}\left(x_{0}\right)+G_{\mathrm{c}}\left(x_{0}\right) u_{\mathrm{c}}\left(t_{0}\right)\right] } \\
= & s_{\mathrm{c} i}\left(u_{\mathrm{c} i}\left(t_{0}\right), y_{\mathrm{c} i}\left(t_{0}\right)\right)+w_{\mathrm{c} i}\left(V_{\mathrm{s}}\left(x_{0}\right)\right), \quad x_{0} \notin \mathscr{Z}_{x}, u_{\mathrm{c}}\left(t_{0}\right) \in \mathbb{R}^{m_{\mathrm{c}}} .
\end{aligned}
$$

Since $x_{0} \notin \mathscr{L}_{x}$ is arbitrary, it follows that

$$
\begin{aligned}
v_{\mathrm{s} i}^{\prime}(x) & {\left[\mathscr{F}_{\mathrm{c}}(x)+G_{\mathrm{c}}(x) u_{\mathrm{c}}\right] } \\
= & w_{\mathrm{c} i}\left(V_{\mathrm{s}}(x)\right)+u_{\mathrm{c}}^{\mathrm{T}} \hat{R}_{\mathrm{c} i} u_{\mathrm{c}}+2 y_{\mathrm{c}}^{\mathrm{T}} \hat{S}_{\mathrm{c} i} u_{\mathrm{c}}+y_{\mathrm{c}}^{\mathrm{T}} \hat{Q}_{\mathrm{c} i} y_{\mathrm{c}} \\
= & w_{\mathrm{c} i}\left(V_{\mathrm{s}}(x)\right)+h_{\mathrm{c}}^{\mathrm{T}}(x) \hat{Q}_{\mathrm{c} i} h_{\mathrm{c}}(x)+2 h_{\mathrm{c}}^{\mathrm{T}}(x)\left(\hat{Q}_{\mathrm{c} i} J_{\mathrm{c}}(x)+\hat{S}_{\mathrm{c} i}\right) u_{\mathrm{c}} \\
& +u_{\mathrm{c}}^{\mathrm{T}}\left(\hat{R}_{\mathrm{c} i}+\hat{S}_{\mathrm{c} i}^{\mathrm{T}} J_{\mathrm{c}}(x)+J_{\mathrm{c}}^{\mathrm{T}}(x) \hat{S}_{\mathrm{c} i}+J_{\mathrm{c}}^{\mathrm{T}}(x) \hat{Q}_{\mathrm{c} i} J_{\mathrm{c}}(x)\right) u_{\mathrm{c}}, \quad x \in \mathbb{R}^{n}, u_{\mathrm{c}} \in \mathbb{R}^{m_{\mathrm{c}}} .
\end{aligned}
$$

Now, equating coefficients of equal powers yields (4.26), (4.27), and (4.28). Next, it follows from (4.33) with $k=1$ that

$$
v_{\mathrm{s} i}\left(x\left(t_{1}\right)+\mathscr{F}_{\mathrm{d}}\left(x\left(t_{1}\right)\right)+G_{\mathrm{d}}\left(x\left(t_{1}\right)\right) u_{\mathrm{d}}\left(t_{1}\right)\right)=v_{\mathrm{s} i}\left(x\left(t_{1}\right)\right)+s_{\mathrm{d} i}\left(u_{\mathrm{d} i}\left(t_{1}\right), y_{\mathrm{d} i}\left(t_{1}\right)\right) .
$$

Now, since the continuous-time dynamics (3.1) are Lipschitz, it follows that for arbitrary $x \in \mathscr{E}_{x}$, there exists $x_{0} \notin \mathscr{E}_{x}$ such that $x\left(t_{1}\right)=x$. Hence, it follows from (4.37) that

$$
\begin{aligned}
v_{\mathrm{s} i}(x+ & \left.\mathscr{F}_{\mathrm{d}}(x)+G_{\mathrm{d}}(x) u_{\mathrm{d}}\right) \\
= & v_{\mathrm{s} i}(x)+u_{\mathrm{d}}^{\mathrm{T}} \hat{R}_{\mathrm{d} i} u_{\mathrm{d}}+2 y_{\mathrm{d}}^{\mathrm{T}} \hat{S}_{\mathrm{d} i} u_{\mathrm{d}}+y_{\mathrm{d}}^{\mathrm{T}} \hat{Q}_{\mathrm{d} i} y_{\mathrm{d}} \\
= & v_{\mathrm{s} i}(x)+h_{\mathrm{d}}^{\mathrm{T}}(x) \hat{Q}_{\mathrm{d} i} h_{\mathrm{d}}(x)+2 h_{\mathrm{d}}^{\mathrm{T}}(x)\left(\hat{Q}_{\mathrm{d} i} J_{\mathrm{d}}(x)+\hat{S}_{\mathrm{d} i}\right) u_{\mathrm{d}} \\
& +u_{\mathrm{d}}^{\mathrm{T}}\left(\hat{R}_{\mathrm{d} i}+\hat{S}_{\mathrm{d} i}^{\mathrm{T}} J_{\mathrm{d}}(x)+J_{\mathrm{d}}^{\mathrm{T}}(x) \hat{S}_{\mathrm{d} i}+J_{\mathrm{d}}^{\mathrm{T}}(x) \hat{Q}_{\mathrm{d} i} J_{\mathrm{d}}(x)\right) u_{\mathrm{d}}, \quad x \in \mathbb{R}^{n}, u_{\mathrm{d}} \in \mathbb{R}^{m_{\mathrm{d}}} .
\end{aligned}
$$

Since the right-hand side of (4.38) is quadratic in $u_{\mathrm{d}}$, it follows that $v_{s i}\left(x+\mathscr{F}_{\mathrm{d}}(x)+\right.$ $\left.G_{\mathrm{d}}(x) u_{\mathrm{d}}\right)$ is quadratic in $u_{\mathrm{d}}$ and hence there exist $P_{1 i}: \mathbb{R}^{n} \rightarrow \mathbb{R}^{1 \times m_{\mathrm{d}}}$ and $P_{2 i}: \mathbb{R}^{n} \rightarrow \mathbb{N}^{m_{\mathrm{d}}}$ such that

$$
\begin{aligned}
v_{\mathrm{s} i}(x+ & \left.+\mathscr{F}_{\mathrm{d}}(x)+G_{\mathrm{d}}(x) u_{\mathrm{d}}\right) \\
& =v_{\mathrm{s} i}\left(x+\mathscr{F}_{\mathrm{d}}(x)\right)+P_{1 i}(x) u_{\mathrm{d}}+u_{\mathrm{d}}^{\mathrm{T}} P_{2 i}(x) u_{\mathrm{d}}, \quad x \in \mathbb{R}^{n}, u_{\mathrm{d}} \in \mathbb{R}^{m_{\mathrm{d}}} .
\end{aligned}
$$

Now, using (4.39) and equating coefficients of equal powers in (4.38) yields (4.29), (4.30), and (4.31).

\section{Stability of feedback interconnections of large-scale impulsive dynamical systems}

In this section, we consider stability of feedback interconnections of large-scale impulsive dynamical systems. Specifically, for the large-scale impulsive dynamical system $\mathscr{G}$ given by 
(3.1), (3.2), (3.3), and (3.4), we consider either a dynamic or a static large-scale feedback system $\mathscr{G}_{\mathrm{c}}$. Then by appropriately combining vector storage functions for each system, we show stability of the feedback interconnection. We begin by considering the large-scale impulsive dynamical system (3.1), (3.2), (3.3), and (3.4) with the large-scale feedback system $\mathscr{G}_{\mathrm{c}}$ given by

$$
\begin{aligned}
\dot{x}_{\mathrm{c}}(t) & =F_{\mathrm{cc}}\left(x_{\mathrm{c}}(t), u_{\mathrm{cc}}(t)\right), & x_{\mathrm{c}}\left(t_{0}\right)=x_{\mathrm{c} 0}, & \left(x_{\mathrm{c}}(t), u_{\mathrm{cc}}(t)\right) \notin \mathscr{E}_{\mathrm{c}}, \\
\Delta x_{\mathrm{c}}(t) & =F_{\mathrm{dc}}\left(x_{\mathrm{c}}(t), u_{\mathrm{dc}}(t)\right), & & \left(x_{\mathrm{c}}(t), u_{\mathrm{cc}}(t)\right) \in \mathscr{E}_{\mathrm{c}}, \\
y_{\mathrm{cc}}(t) & =H_{\mathrm{cc}}\left(x_{\mathrm{c}}(t), u_{\mathrm{cc}}(t)\right), & & \left(x_{\mathrm{c}}(t), u_{\mathrm{cc}}(t)\right) \notin \mathscr{E}_{\mathrm{c}}, \\
y_{\mathrm{dc}}(t) & =H_{\mathrm{dc}}\left(x_{\mathrm{c}}(t), u_{\mathrm{dc}}(t)\right), & & \left(x_{\mathrm{c}}(t), u_{\mathrm{cc}}(t)\right) \in \mathscr{E}_{\mathrm{c}},
\end{aligned}
$$

where $F_{\mathrm{cc}}: \mathbb{R}^{n_{\mathrm{c}}} \times \boldsymbol{U}_{\mathrm{cc}} \rightarrow \mathbb{R}^{n_{\mathrm{c}}}, F_{\mathrm{dc}}: \mathbb{R}^{n_{\mathrm{c}}} \times \boldsymbol{U}_{\mathrm{dc}} \rightarrow \mathbb{R}^{n_{\mathrm{c}}}, H_{\mathrm{cc}}: \mathbb{R}^{n_{\mathrm{c}}} \times \boldsymbol{U}_{\mathrm{cc}} \rightarrow \mathscr{Y}_{\mathrm{cc}}, H_{\mathrm{dc}}: \mathbb{R}^{n_{\mathrm{c}}} \times$ $u_{\mathrm{dc}} \rightarrow \mathscr{Y}_{\mathrm{dc}}, F_{\mathrm{cc}} \triangleq\left[F_{\mathrm{cc} 1}^{\mathrm{T}}, \ldots, F_{\mathrm{cc} q}^{\mathrm{T}}\right]^{\mathrm{T}}, F_{\mathrm{dc}} \triangleq\left[F_{\mathrm{dcl}}^{\mathrm{T}}, \ldots, F_{\mathrm{dc} q}^{\mathrm{T}}\right]^{\mathrm{T}}, H_{\mathrm{cc}} \triangleq\left[H_{\mathrm{cc} 1}^{\mathrm{T}}, \ldots, H_{\mathrm{cc} q}^{\mathrm{T}}\right]^{\mathrm{T}}, H_{\mathrm{dc}} \triangleq$ $\left[H_{\mathrm{dcl}}^{\mathrm{T}}, \ldots, H_{\mathrm{dc} q}^{\mathrm{T}}\right]^{\mathrm{T}}, \boldsymbol{U}_{\mathrm{cc}} \subseteq \mathbb{R}^{l_{\mathrm{c}}}, \boldsymbol{U}_{\mathrm{dc}} \subseteq \mathbb{R}^{l_{\mathrm{d}}}, \mathscr{Y}_{\mathrm{cc}} \subseteq \mathbb{R}^{m_{\mathrm{c}}}$, and $\mathscr{Y}_{\mathrm{dc}} \subseteq \mathbb{R}^{m_{\mathrm{d}}}$. Moreover, for all $i=$ $1, \ldots, q$, we assume that

$$
\begin{aligned}
F_{\mathrm{cc} i}\left(x_{\mathrm{c}}, u_{\mathrm{cc} i}\right) & =f_{\mathrm{cc} i}\left(x_{\mathrm{c} i}\right)+\Phi_{\mathrm{cc} i}\left(x_{\mathrm{c}}\right)+G_{\mathrm{cc} i}\left(x_{\mathrm{c} i}\right) u_{\mathrm{cc} i}, \\
F_{\mathrm{dc} i}\left(x_{\mathrm{c}}, u_{\mathrm{d} c i}\right) & =f_{\mathrm{dc} i}\left(x_{\mathrm{c} i}\right)+\Phi_{\mathrm{d} c i}\left(x_{\mathrm{c}}\right)+G_{\mathrm{d} c i}\left(x_{\mathrm{c} i}\right) u_{\mathrm{d} c i}, \\
H_{\mathrm{cc} i}\left(x_{\mathrm{c} i}, u_{\mathrm{cc} i}\right) & =h_{\mathrm{cc} i}\left(x_{\mathrm{c} i}\right)+J_{\mathrm{cc} i}\left(x_{\mathrm{c} i}\right) u_{\mathrm{cc} i}, \\
H_{\mathrm{d} c i}\left(x_{\mathrm{c} i}, u_{\mathrm{d} c i}\right) & =h_{\mathrm{dc} i}\left(x_{\mathrm{c} i}\right)+J_{\mathrm{dc} i}\left(x_{\mathrm{c} i}\right) u_{\mathrm{dc} i},
\end{aligned}
$$

where $u_{\mathrm{cc} i} \in \mathcal{U}_{\mathrm{cc} i} \subseteq \mathbb{R}^{l_{c i}}, u_{\mathrm{d} c i} \in \mathcal{U}_{\mathrm{dci} i} \subseteq \mathbb{R}^{l_{\mathrm{d} i}}, y_{\mathrm{cc} i} \triangleq H_{\mathrm{cc} i}\left(x_{\mathrm{ci}}, u_{\mathrm{cc} i}\right) \in \mathscr{Y}_{\mathrm{cc} i} \subseteq \mathbb{R}^{m_{\mathrm{c} i}}, y_{\mathrm{d} c i} \triangleq$ $H_{\mathrm{dci} i}\left(x_{\mathrm{c} i}, u_{\mathrm{dc} i}\right) \in \mathscr{Y}_{\mathrm{dc} i} \subseteq \mathbb{R}^{m_{\mathrm{d} i}}, f_{\mathrm{cci}}: \mathbb{R}^{n_{\mathrm{c} i}} \rightarrow \mathbb{R}^{n_{\mathrm{c} i}}$ and $\mathscr{I}_{\mathrm{cc} i}: \mathbb{R}^{n_{\mathrm{c}}} \rightarrow \mathbb{R}^{n_{\mathrm{c} i}}$ satisfy $f_{\mathrm{cci}}(0)=0$ and $\mathscr{I}_{\mathrm{cci}}(0)=0, f_{\mathrm{dc} i}: \mathbb{R}^{n_{\mathrm{c} i}} \rightarrow \mathbb{R}^{n_{\mathrm{c} i}}, \mathscr{I}_{\mathrm{dci}}: \mathbb{R}^{n_{\mathrm{c}}} \rightarrow \mathbb{R}^{n_{\mathrm{c} i}}, G_{\mathrm{cci} i}: \mathbb{R}^{n_{\mathrm{c} i}} \rightarrow \mathbb{R}^{n_{\mathrm{c} i} \times l_{\mathrm{c} i}}, G_{\mathrm{dc} i}: \mathbb{R}^{n_{\mathrm{c} i}} \rightarrow \mathbb{R}^{n_{\mathrm{c} i} \times l_{\mathrm{d} i}}$, $h_{\mathrm{cci}}: \mathbb{R}^{n_{\mathrm{c} i}} \rightarrow \mathbb{R}^{m_{\mathrm{ci}}}$ and satisfies $h_{\mathrm{cci}}(0)=0, h_{\mathrm{dci}}: \mathbb{R}^{n_{\mathrm{c} i}} \rightarrow \mathbb{R}^{m_{\mathrm{d} i}}, J_{\mathrm{cci}}: \mathbb{R}^{n_{\mathrm{c} i}} \rightarrow \mathbb{R}^{m_{\mathrm{c} i} \times l_{c i}}, J_{\mathrm{dci}}: \mathbb{R}^{n_{\mathrm{c} i}} \rightarrow$ $\mathbb{R}^{m_{\mathrm{di}} \times l_{\mathrm{d} i}}$, and $\sum_{i=1}^{q} n_{\mathrm{c} i}=n_{\mathrm{c}}$. Furthermore, we define the composite input and composite output for the system $\mathscr{G}_{\mathrm{c}}$ as $u_{\mathrm{cc}} \triangleq\left[u_{\mathrm{cc} 1}^{\mathrm{T}}, \ldots, u_{\mathrm{cc} q}^{\mathrm{T}}\right]^{\mathrm{T}}, u_{\mathrm{dc}} \triangleq\left[u_{\mathrm{dcl}}^{\mathrm{T}}, \ldots, u_{\mathrm{dc} q}^{\mathrm{T}}\right]^{\mathrm{T}}, y_{\mathrm{cc}} \triangleq\left[y_{\mathrm{cc} 1}^{\mathrm{T}}, \ldots\right.$, $\left.y_{c c q}^{\mathrm{T}}\right]^{\mathrm{T}}$, and $y_{\mathrm{dc}} \triangleq\left[y_{\mathrm{dcl}}^{\mathrm{T}}, \ldots, y_{\mathrm{dcq}}^{\mathrm{T}}\right]^{\mathrm{T}}$, respectively. In this case, $\boldsymbol{\vartheta}_{\mathrm{cc}}=u_{\mathrm{ccl}} \times \cdots \times \boldsymbol{u}_{\mathrm{cc} q}, \boldsymbol{u}_{\mathrm{dc}}$ $=\boldsymbol{U}_{\mathrm{dc1}} \times \cdots \times \boldsymbol{U}_{\mathrm{dcq}}, \mathscr{y}_{\mathrm{cc}}=\mathscr{y}_{\mathrm{ccl}} \times \cdots \times \mathscr{Y}_{\mathrm{cc} q}$, and $\mathscr{y}_{\mathrm{dc}}=\mathscr{y}_{\mathrm{dc1}} \times \cdots \times \mathscr{Y}_{\mathrm{dcq}}$. Note that with the feedback interconnection given by Figure $5.1,\left(u_{\mathrm{cc}}, u_{\mathrm{dc}}\right)=\left(y_{\mathrm{c}}, y_{\mathrm{d}}\right)$ and $\left(y_{\mathrm{cc}}, y_{\mathrm{dc}}\right)=$ $\left(-u_{\mathrm{c}},-u_{\mathrm{d}}\right)$. We assume that the negative feedback interconnection of $\varphi_{\text {and }} \varphi_{\mathrm{c}}$ is well posed; that is, $\operatorname{det}\left(I_{m_{\mathrm{c} i}}+J_{\mathrm{cci} i}\left(x_{\mathrm{c} i}\right) J_{\mathrm{c} i}\left(x_{i}\right)\right) \neq 0$, $\operatorname{det}\left(I_{m_{\mathrm{d} i}}+J_{\mathrm{dc} i}\left(x_{\mathrm{c} i}\right) J_{\mathrm{d} i}\left(x_{i}\right)\right) \neq 0$, for all $x_{i} \in \mathbb{R}^{n_{i}}$, $x_{\mathrm{c} i} \in \mathbb{R}^{n_{\mathrm{c} i}}$, and $i=1, \ldots, q$. Next, we assume that the set $\mathscr{L}_{\mathrm{c}} \triangleq \mathscr{E}_{\mathrm{c} x_{\mathrm{c}}} \times \mathscr{E}_{\mathrm{c} u_{\mathrm{cc}}}=\left\{\left(x_{\mathrm{c}}, u_{\mathrm{cc}}\right)\right.$ : $\left.\mathscr{X}_{\mathrm{c}}\left(x_{\mathrm{c}}, u_{\mathrm{cc}}\right)=0\right\}$, where $\mathscr{X}_{\mathrm{c}}: \mathbb{R}^{n_{\mathrm{c}}} \times \mathcal{U}_{\mathrm{cc}} \rightarrow \mathbb{R}$, and define the closed-loop resetting set

$$
\tilde{\mathscr{E}}_{\tilde{x}} \triangleq \mathscr{E}_{x} \times \mathscr{L}_{\mathrm{cx}} \cup\left\{\left(x, x_{\mathrm{c}}\right):\left(\mathscr{L}_{\mathrm{cc}}\left(x, x_{\mathrm{c}}\right), \mathscr{L}_{\mathrm{c}}\left(x, x_{\mathrm{c}}\right)\right) \in \mathscr{E}_{\mathrm{c} u_{\mathrm{cc}}} \times \mathscr{L}_{u_{\mathrm{c}}}\right\}
$$

where $\mathscr{L}_{\mathrm{cc}}(\cdot, \cdot)$ and $\mathscr{L}_{\mathrm{c}}(\cdot, \cdot)$ are functions of $x$ and $x_{\mathrm{c}}$ arising from the algebraic loops due to $u_{\mathrm{cc}}$ and $u_{\mathrm{c}}$, respectively. Note that since the feedback interconnection of $\varphi_{\mathcal{G}}$ and $\mathscr{G}_{\mathrm{c}}$ is well posed, it follows that $\tilde{\mathscr{E}}_{\tilde{x}}$ is well defined and depends on the closed-loop states $\tilde{x} \triangleq\left[\begin{array}{ll}x^{\mathrm{T}} & x_{\mathrm{c}}^{\mathrm{T}}\end{array}\right]^{\mathrm{T}}$. Furthermore, we assume that for the large-scale systems $\mathscr{G}_{\mathrm{G}}$ and $\mathscr{G}_{\mathrm{c}}$, the conditions of Theorem 3.9 are satisfied; that is, if $V_{\mathrm{s}}(x), x \in \mathbb{R}^{n}$, and $V_{\mathrm{cs}}\left(x_{\mathrm{c}}\right), x_{\mathrm{c}} \in \mathbb{R}^{n_{\mathrm{c}}}$, are vector storage functions for $\mathscr{G}_{\text {and }} \mathscr{G}_{\mathrm{c}}$, respectively, then there exist $p \in \mathbb{R}_{+}^{q}$ and $p_{\mathrm{c}} \in \mathbb{R}_{+}^{q}$ 


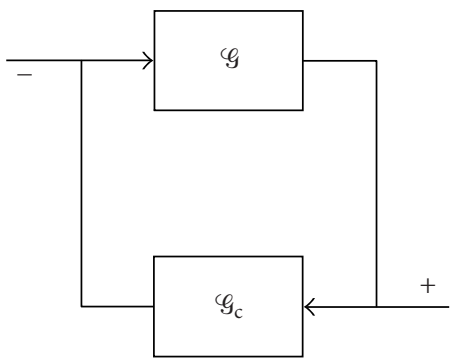

Figure 5.1. Feedback interconnection of large-scale systems $\mathscr{G}_{\text {and }} \mathscr{G}_{\mathrm{c}}$.

such that the functions $v_{\mathrm{s}}(x)=p^{\mathrm{T}} V_{\mathrm{s}}(x), x \in \mathbb{R}^{n}$, and $v_{\mathrm{cs}}\left(x_{\mathrm{c}}\right)=p_{\mathrm{c}}^{\mathrm{T}} V_{\mathrm{cs}}\left(x_{\mathrm{c}}\right), x_{\mathrm{c}} \in \mathbb{R}^{n_{\mathrm{c}}}$, are positive-definite. The following result gives sufficient conditions for Lyapunov and asymptotic stability of the feedback interconnection given by Figure 5.1. For the statement of this result, let $\mathscr{T}_{x_{0}, u_{c}}^{c}$ denote the set of resetting times of $\mathscr{G}$, let $\mathscr{T}_{x_{0}, u_{c}}$ denote the complement of $\mathscr{T}_{x_{0}, u_{\mathrm{c}}}^{\mathrm{c}}$, that is, $[0, \infty) \backslash \mathscr{T}_{x_{0}, u_{\mathrm{c}}}^{\mathrm{c}}$, let $\mathscr{T}_{x_{\mathrm{c} 0}, u_{\mathrm{cc}}}^{\mathrm{c}}$ denote the set of resetting times of $\mathscr{G}_{\mathrm{c}}$, and let $\mathscr{T}_{x_{\mathrm{c} 0}, u_{\mathrm{cc}}}$ denote the complement of $\mathscr{T}_{x_{\mathrm{c} 0}, u_{\mathrm{cc}}}^{\mathrm{c}}$, that is, $[0, \infty) \backslash \mathscr{T}_{x_{\mathrm{c} 0}, u_{\mathrm{cc}}}^{\mathrm{c}}$.

Theorem 5.1. Consider the large-scale impulsive dynamical systems $\mathscr{G}_{\text {and }} \mathscr{G}_{\mathrm{c}}$ given by (3.1), (3.2), (3.3), and (3.4) and (5.1), respectively. Assume that $\mathscr{G}_{\mathrm{G}}$ and $\mathscr{G}_{\mathrm{c}}$ are vector dissipative with respect to the vector hybrid supply rates $\left(S_{\mathrm{c}}\left(u_{\mathrm{c}}, y_{\mathrm{c}}\right), S_{\mathrm{d}}\left(u_{\mathrm{d}}, y_{\mathrm{d}}\right)\right)$ and $\left(S_{\mathrm{cc}}\left(u_{\mathrm{cc}}, y_{\mathrm{cc}}\right), S_{\mathrm{dc}}\left(u_{\mathrm{dc}}, y_{\mathrm{dc}}\right)\right)$, and with continuously differentiable vector storage functions $V_{\mathrm{s}}(\cdot)$ and $V_{\mathrm{cs}}(\cdot)$ and dissipation matrices $W \in \mathbb{R}^{q \times q}$ and $W_{\mathrm{c}} \in \mathbb{R}^{q \times q}$, respectively.

(i) If there exists $\Sigma \triangleq \operatorname{diag}\left[\sigma_{1}, \ldots, \sigma_{q}\right]>0$ such that $S_{\mathrm{c}}\left(u_{\mathrm{c}}, y_{\mathrm{c}}\right)+\Sigma S_{\mathrm{cc}}\left(u_{\mathrm{cc}}, y_{\mathrm{cc}}\right) \leq \leq 0$, $S_{\mathrm{d}}\left(u_{\mathrm{d}}, y_{\mathrm{d}}\right)+\Sigma S_{\mathrm{dc}}\left(u_{\mathrm{dc}}, y_{\mathrm{dc}}\right) \leq \leq 0$, and $\tilde{W} \in \mathbb{R}^{q \times q}$ is semistable (resp., asymptotically stable), where $\tilde{W}_{(i, j)} \triangleq \max \left\{W_{(i, j)},\left(\sum W_{\mathrm{c}} \Sigma^{-1}\right)_{(i, j)}\right\}=\max \left\{W_{(i, j)},\left(\sigma_{i} / \sigma_{j}\right) W_{c(i, j)}\right\}$, $i, j=1, \ldots, q$, then the negative feedback interconnection of $\mathscr{G}_{\text {and }} \mathscr{G}_{\mathrm{c}}$ is Lyapunov (resp., asymptotically) stable.

(ii) Let $Q_{\mathrm{c} i} \in \mathbb{S}^{l_{\mathrm{c} i}}, S_{\mathrm{c} i} \in \mathbb{R}^{l_{\mathrm{c} i} \times m_{\mathrm{c} i}}, R_{\mathrm{c} i} \in \mathbb{S}^{m_{\mathrm{c} i}}, Q_{\mathrm{d} i} \in \mathbb{S}^{l_{\mathrm{d} i}}, S_{\mathrm{d} i} \in \mathbb{R}^{l_{\mathrm{d} i} \times m_{\mathrm{d} i}}, R_{\mathrm{d} i} \in \mathbb{S}^{m_{\mathrm{d} i}}, Q_{\mathrm{cc} i} \in$ $\mathbb{S}^{m_{\mathrm{c} i}}, S_{\mathrm{cc} i} \in \mathbb{R}^{m_{\mathrm{c} i} \times l_{c i}}, R_{\mathrm{cci}} \in \mathbb{S}^{l_{c i}}, Q_{\mathrm{dc} i} \in \mathbb{S}^{m_{\mathrm{d} i}}, S_{\mathrm{d} c i} \in \mathbb{R}^{m_{\mathrm{d} i} \times l_{\mathrm{d} i}}$, and $R_{\mathrm{d} c i} \in \mathbb{S}^{l_{\mathrm{d} i}}$, and suppose $S_{\mathrm{c}}\left(u_{\mathrm{c}}, y_{\mathrm{c}}\right)=\left[s_{\mathrm{c} 1}\left(u_{\mathrm{c} 1}, y_{\mathrm{c} 1}\right), \ldots, s_{\mathrm{c} q}\left(u_{\mathrm{c} q}, y_{\mathrm{c} q}\right)\right]^{\mathrm{T}}, S_{\mathrm{d}}\left(u_{\mathrm{d}}, y_{\mathrm{d}}\right)=\left[s_{\mathrm{d} 1}\left(u_{\mathrm{d} 1}, y_{\mathrm{d} 1}\right), \ldots, s_{\mathrm{d} q}\left(u_{\mathrm{d} q}\right.\right.$, $\left.\left.y_{\mathrm{d} q}\right)\right]^{\mathrm{T}}, \quad S_{\mathrm{cc}}\left(u_{\mathrm{cc}}, y_{\mathrm{cc}}\right)=\left[s_{\mathrm{ccl}}\left(u_{\mathrm{ccl}}, y_{\mathrm{ccl}}\right), \ldots, s_{\mathrm{cc} q}\left(u_{\mathrm{cc} q}, y_{\mathrm{cc} q}\right)\right]^{\mathrm{T}}$, and $S_{\mathrm{dc}}\left(u_{\mathrm{dc}}, y_{\mathrm{dc}}\right)=$ $\left[s_{\mathrm{dcl}}\left(u_{\mathrm{dcl}}, y_{\mathrm{dcl}}\right), \ldots, s_{\mathrm{dc} q}\left(u_{\mathrm{dc} q}, y_{\mathrm{dc} q}\right)\right]^{\mathrm{T}}$, where $s_{\mathrm{c} i}\left(u_{\mathrm{c} i}, y_{\mathrm{c} i}\right)=u_{\mathrm{c} i}^{\mathrm{T}} R_{\mathrm{c} i} u_{\mathrm{c} i}+2 y_{\mathrm{ci}}^{\mathrm{T}} S_{\mathrm{c} i} u_{\mathrm{c} i}+$ $y_{\mathrm{c} i}^{\mathrm{T}} Q_{\mathrm{c} i} y_{\mathrm{c} i}, s_{\mathrm{d} i}\left(u_{\mathrm{d} i}, y_{\mathrm{d} i}\right)=u_{\mathrm{d} i}^{\mathrm{T}} R_{\mathrm{d} i} u_{\mathrm{d} i}+2 y_{\mathrm{d} i}^{\mathrm{T}} S_{\mathrm{d} i} u_{\mathrm{d} i}+y_{\mathrm{d} i}^{\mathrm{T}} Q_{\mathrm{d} i} y_{\mathrm{d} i}, s_{\mathrm{cc} i}\left(u_{\mathrm{cc} i}, y_{\mathrm{cc} i}\right)=u_{\mathrm{cc} i}^{\mathrm{T}} R_{\mathrm{cc} i} u_{\mathrm{cc} i}$ $+2 y_{\mathrm{cci}}^{\mathrm{T}} S_{\mathrm{cc} i} u_{\mathrm{cc} i}+y_{\mathrm{cci}}^{\mathrm{T}} Q_{\mathrm{cc} i} y_{\mathrm{cci}}$, and $s_{\mathrm{dci}}\left(u_{\mathrm{dc} i}, y_{\mathrm{dci}}\right)=u_{\mathrm{dci}}^{\mathrm{T}} R_{\mathrm{dc} i} u_{\mathrm{dc} i}+2 y_{\mathrm{dc} i}^{\mathrm{T}} S_{\mathrm{d} c i} u_{\mathrm{dc} i}+$ $y_{\mathrm{dc} i}^{\mathrm{T}} Q_{\mathrm{dc} i} y_{\mathrm{dc} i}, i=1, \ldots, q$. If there exists $\Sigma \triangleq \operatorname{diag}\left[\sigma_{1}, \ldots, \sigma_{q}\right]>0$ such that for all $i=$ $1, \ldots, q$,

$$
\begin{gathered}
\tilde{Q}_{\mathrm{c} i} \triangleq\left[\begin{array}{cc}
Q_{\mathrm{c} i}+\sigma_{i} R_{\mathrm{cci}} & -S_{\mathrm{c} i}+\sigma_{i} S_{\mathrm{cc} i}^{\mathrm{T}} \\
-S_{\mathrm{c} i}^{\mathrm{T}}+\sigma_{i} S_{\mathrm{cc} i} & R_{\mathrm{c} i}+\sigma_{i} Q_{\mathrm{cc} i}
\end{array}\right] \leq 0, \\
\tilde{Q}_{\mathrm{d} i} \triangleq\left[\begin{array}{cc}
Q_{\mathrm{d} i}+\sigma_{i} R_{\mathrm{dci} i} & -S_{\mathrm{d} i}+\sigma_{i} S_{\mathrm{d} c i}^{\mathrm{T}} \\
-S_{\mathrm{d} i}^{\mathrm{T}}+\sigma_{i} S_{\mathrm{d} c i} & R_{\mathrm{d} i}+\sigma_{i} Q_{\mathrm{d} c i}
\end{array}\right] \leq 0,
\end{gathered}
$$


and $\tilde{W} \in \mathbb{R}^{q \times q}$ is semistable (resp., asymptotically stable), where $\tilde{W}_{(i, j)} \triangleq \max \left\{W_{(i, j)}\right.$, $\left.\left(\Sigma W_{\mathrm{c}} \Sigma^{-1}\right)_{(i, j)}\right\}=\max \left\{W_{(i, j)},\left(\sigma_{i} / \sigma_{j}\right) W_{\mathrm{c}(i, j)}\right\}, i, j=1, \ldots, q$, then the negative feedback interconnection of $\mathscr{G}_{\mathrm{S}}$ and $\mathscr{G}_{\mathrm{c}}$ is Lyapunov (resp., asymptotically) stable.

Proof. Let $\tilde{\mathscr{T}}^{\mathrm{c}} \triangleq \mathscr{T}_{x_{0}, u_{\mathrm{c}}}^{\mathrm{c}} \cup \mathscr{T}_{x_{\mathrm{c} 0}, u_{\mathrm{cc}}}^{\mathrm{c}}$ and $t_{k} \in \tilde{\mathscr{T}}^{\mathrm{c}}, k \in \overline{\mathbb{Z}}_{+}$. First, note that it follows from assumptions (A1) and (A2) that the resetting times $t_{k}\left(=\tau_{k}\left(\tilde{x}_{0}\right)\right)$ for the feedback system are well defined and distinct for every closed-loop trajectory.

(i) Consider the vector Lyapunov function candidate $V\left(x, x_{\mathrm{c}}\right)=V_{\mathrm{s}}(x)+\Sigma V_{\mathrm{cs}}\left(x_{\mathrm{c}}\right)$, $\left(x, x_{\mathrm{c}}\right) \in \mathbb{R}^{n} \times \mathbb{R}^{n_{\mathrm{c}}}$, and note that the corresponding vector Lyapunov derivative of $V\left(x, x_{\mathrm{c}}\right)$ along the state trajectories $\left(x(t), x_{\mathrm{c}}(t)\right), t \in\left(t_{k}, t_{k+1}\right)$, is given by

$$
\begin{aligned}
\dot{V}\left(x(t), x_{\mathrm{c}}(t)\right)= & \dot{V}_{\mathrm{s}}(x(t))+\sum \dot{V}_{\mathrm{cs}}\left(x_{\mathrm{c}}(t)\right) \\
\leq \leq & S_{\mathrm{c}}\left(u_{\mathrm{c}}(t), y_{\mathrm{c}}(t)\right)+\Sigma S_{\mathrm{cc}}\left(u_{\mathrm{cc}}(t), y_{\mathrm{cc}}(t)\right) \\
& +W V_{\mathrm{s}}(x(t))+\sum W_{\mathrm{c}} V_{\mathrm{cs}}\left(x_{\mathrm{c}}(t)\right) \\
\leq \leq & W V_{\mathrm{s}}(x(t))+\sum W_{\mathrm{c}} \Sigma^{-1} \Sigma V_{\mathrm{cs}}\left(x_{\mathrm{c}}(t)\right) \\
\leq \leq & \tilde{W}\left(V_{\mathrm{s}}(x(t))+\sum V_{\mathrm{cs}}\left(x_{\mathrm{c}}(t)\right)\right) \\
= & \tilde{W} V\left(x(t), x_{\mathrm{c}}(t)\right), \quad\left(x(t), x_{\mathrm{c}}(t)\right) \notin \tilde{\mathscr{E}}_{\tilde{x}},
\end{aligned}
$$

and the Lyapunov difference of $V\left(x, x_{\mathrm{c}}\right)$ at the resetting times $t_{k}, k \in \overline{\mathbb{Z}}_{+}$, is given by

$$
\begin{aligned}
\Delta V\left(x\left(t_{k}\right), x_{\mathrm{c}}\left(t_{k}\right)\right) & =\Delta V_{\mathrm{s}}\left(x\left(t_{k}\right)\right)+\Sigma \Delta V_{\mathrm{cs}}\left(x_{\mathrm{c}}\left(t_{k}\right)\right) \\
& \leq \leq S_{\mathrm{d}}\left(u_{\mathrm{d}}\left(t_{k}\right), y_{\mathrm{d}}\left(t_{k}\right)\right)+\Sigma S_{\mathrm{dc}}\left(u_{\mathrm{dc}}\left(t_{k}\right), y_{\mathrm{dc}}\left(t_{k}\right)\right) \\
& \leq \leq 0, \quad\left(x(t), x_{\mathrm{c}}(t)\right) \in \tilde{\mathscr{E}}_{\tilde{x}} .
\end{aligned}
$$

Next, since for $V_{\mathrm{s}}(x), x \in \mathbb{R}^{n}$, and $V_{\mathrm{cs}}\left(x_{\mathrm{c}}\right), x_{\mathrm{c}} \in \mathbb{R}^{n_{\mathrm{c}}}$, there exist, by assumption, $p \in \mathbb{R}_{+}^{q}$ and $p_{\mathrm{c}} \in \mathbb{R}_{+}^{q}$ such that the functions $v_{\mathrm{s}}(x)=p^{\mathrm{T}} V_{\mathrm{s}}(x), x \in \mathbb{R}^{n}$, and $v_{\mathrm{cs}}\left(x_{\mathrm{c}}\right)=p_{\mathrm{c}}^{\mathrm{T}} V_{\mathrm{cs}}\left(x_{\mathrm{c}}\right)$, $x_{\mathrm{c}} \in \mathbb{R}^{n_{\mathrm{c}}}$, are positive-definite, and noting that $v_{\mathrm{cs}}\left(x_{\mathrm{c}}\right) \leq \max _{i=1, \ldots, q}\left\{p_{\mathrm{c} i}\right\} \mathbf{e}^{\mathrm{T}} V_{\mathrm{cs}}\left(x_{\mathrm{c}}\right)$, where $p_{c i}$ is the $i$ th element of $p_{\mathrm{c}}$ and $\mathbf{e} \triangleq[1, \ldots, 1]^{\mathrm{T}}$, it follows that $\mathbf{e}^{\mathrm{T}} V_{\mathrm{cs}}\left(x_{\mathrm{c}}\right), x_{\mathrm{c}} \in \mathbb{R}^{n_{\mathrm{c}}}$, is positive-definite. Now, since $\min _{i=1, \ldots, q}\left\{p_{i} \sigma_{i}\right\} \mathbf{e}^{\mathrm{T}} V_{\mathrm{cs}}\left(x_{\mathrm{c}}\right) \leq p^{\mathrm{T}} \sum V_{\mathrm{cs}}\left(x_{\mathrm{c}}\right)$, it follows that $p^{\mathrm{T}} \sum V_{c s}\left(x_{\mathrm{c}}\right), x_{\mathrm{c}} \in \mathbb{R}^{n_{\mathrm{c}}}$, is positive-definite. Hence, the function $v\left(x, x_{\mathrm{c}}\right)=p^{\mathrm{T}} V\left(x, x_{\mathrm{c}}\right),\left(x, x_{\mathrm{c}}\right)$ $\in \mathbb{R}^{n} \times \mathbb{R}^{n_{\mathrm{c}}}$, is positive-definite. Now, the result is a direct consequence of Theorem 2.7.

(ii) The proof follows from (i) by noting that, for all $i=1, \ldots, q$,

$$
\begin{gathered}
s_{\mathrm{c} i}\left(u_{\mathrm{c} i}, y_{\mathrm{c} i}\right)+\sigma_{i} s_{\mathrm{cc} i}\left(u_{\mathrm{cc} i}, y_{\mathrm{cc} i}\right)=\left[\begin{array}{l}
y_{\mathrm{c}} \\
y_{\mathrm{cc}}
\end{array}\right]^{\mathrm{T}} \tilde{Q}_{\mathrm{c} i}\left[\begin{array}{l}
y_{\mathrm{c}} \\
y_{\mathrm{cc}}
\end{array}\right], \\
s_{\mathrm{d} i}\left(u_{\mathrm{d} i}, y_{\mathrm{d} i}\right)+\sigma_{i} s_{\mathrm{dc} i}\left(u_{\mathrm{dc} i}, y_{\mathrm{dc} i}\right)=\left[\begin{array}{l}
y_{\mathrm{d}} \\
y_{\mathrm{dc}}
\end{array}\right]^{\mathrm{T}} \tilde{Q}_{\mathrm{d} i}\left[\begin{array}{l}
y_{\mathrm{d}} \\
y_{\mathrm{dc}}
\end{array}\right],
\end{gathered}
$$

and hence $S_{\mathrm{c}}\left(u_{\mathrm{c}}, y_{\mathrm{c}}\right)+\Sigma S_{\mathrm{cc}}\left(u_{\mathrm{cc}}, y_{\mathrm{cc}}\right) \leq \leq 0$ and $S_{\mathrm{d}}\left(u_{\mathrm{d}}, y_{\mathrm{d}}\right)+\Sigma S_{\mathrm{dc}}\left(u_{\mathrm{dc}}, y_{\mathrm{dc}}\right) \leq \leq 0$. 
For the next result, note that if the large-scale impulsive dynamical system $\mathscr{G}$ is vector dissipative with respect to the vector hybrid supply rate $\left(S_{\mathrm{c}}\left(u_{\mathrm{c}}, y_{\mathrm{c}}\right), S_{\mathrm{d}}\left(u_{\mathrm{d}}, y_{\mathrm{d}}\right)\right)$, where $s_{\mathrm{c} i}\left(u_{\mathrm{c} i}, y_{\mathrm{c} i}\right)=2 y_{\mathrm{c} i}^{\mathrm{T}} u_{\mathrm{c} i}$ and $s_{\mathrm{d} i}\left(u_{\mathrm{d} i}, y_{\mathrm{d} i}\right)=2 y_{\mathrm{d} i}^{\mathrm{T}} u_{\mathrm{d} i}, i=1, \ldots, q$, then with $\kappa_{\mathrm{c} i}\left(y_{\mathrm{c} i}\right)=-\kappa_{\mathrm{c} i} y_{\mathrm{c} i}$ and $\kappa_{\mathrm{d} i}\left(y_{\mathrm{d} i}\right)=-\kappa_{\mathrm{d} i} y_{\mathrm{d} i}$, where $\kappa_{\mathrm{c} i}>0, \kappa_{\mathrm{d} i}>0, i=1, \ldots, q$, it follows that $s_{\mathrm{c} i}\left(\kappa_{\mathrm{c} i}\left(y_{\mathrm{c} i}\right), y_{\mathrm{c} i}\right)=$ $-\kappa_{\mathrm{c} i} y_{\mathrm{c} i}^{\mathrm{T}} y_{\mathrm{c} i}<0$ and $s_{\mathrm{d} i}\left(\kappa_{\mathrm{d} i}\left(y_{\mathrm{d} i}\right), y_{\mathrm{d} i}\right)=-\kappa_{\mathrm{d} i} y_{\mathrm{d} i}^{\mathrm{T}} y_{\mathrm{d} i}<0, y_{\mathrm{c} i} \neq 0, y_{\mathrm{d} i} \neq 0, i=1, \ldots, q$. Alternatively, if $\mathscr{G}_{\mathrm{i}}$ is vector dissipative with respect to the vector hybrid supply rate $\left(S_{\mathrm{c}}\left(u_{\mathrm{c}}, y_{\mathrm{c}}\right)\right.$, $\left.S_{\mathrm{d}}\left(u_{\mathrm{d}}, y_{\mathrm{d}}\right)\right)$, where $s_{\mathrm{c} i}\left(u_{\mathrm{c} i}, y_{\mathrm{c} i}\right)=\gamma_{\mathrm{c} i}^{2} u_{\mathrm{c} i}^{\mathrm{T}} u_{\mathrm{c} i}-y_{\mathrm{c} i}^{\mathrm{T}} y_{\mathrm{c} i}$ and $s_{\mathrm{d} i}\left(u_{\mathrm{d} i}, y_{\mathrm{d} i}\right)=\gamma_{\mathrm{d} i}^{2} u_{\mathrm{d} i}^{\mathrm{T}} u_{\mathrm{d} i}-y_{\mathrm{d} i}^{\mathrm{T}} y_{\mathrm{d} i}$, where $\gamma_{\mathrm{c} i}>0, \gamma_{\mathrm{d} i}>0, i=1, \ldots, q$, then with $\kappa_{\mathrm{c} i}\left(y_{\mathrm{c} i}\right)=0$ and $\kappa_{\mathrm{d} i}\left(y_{\mathrm{d} i}\right)=0$, it follows that $s_{\mathrm{c} i}\left(\kappa_{\mathrm{c} i}\left(y_{\mathrm{c} i}\right), y_{\mathrm{c} i}\right)=-y_{\mathrm{c} i}^{\mathrm{T}} y_{\mathrm{c} i}<0$ and $s_{\mathrm{d} i}\left(\kappa_{\mathrm{d} i}\left(y_{\mathrm{d} i}\right), y_{\mathrm{d} i}\right)=-y_{\mathrm{d} i}^{\mathrm{T}} y_{\mathrm{d} i}<0, y_{\mathrm{c} i} \neq 0, y_{\mathrm{d} i} \neq 0, i=1, \ldots, q$. Hence, if $\varphi_{\text {is }}$ zero-state observable and the dissipation matrix $W$ is such that there exist $\alpha \geq 0$ and $p \in \mathbb{R}_{+}^{q}$ such that (2.2) holds, then it follows from Theorem 3.9 that (scalar) storage functions of the form $v_{s}(x)=p^{\mathrm{T}} V_{s}(x), x \in \mathbb{R}^{n}$, where $V_{s}(\cdot)$ is a vector storage function for $\mathscr{G}$, are positive-definite. If $\mathscr{G}$ is exponentially vector dissipative, then $p$ is positive.

Corollary 5.2. Consider the large-scale impulsive dynamical systems $\mathscr{G}_{\text {and }} \mathscr{G}_{\mathrm{c}}$ given by (3.1), (3.2), (3.3), and (3.4) and (5.1), respectively. Assume that $\varphi_{G}$ and $\varphi_{\mathrm{c}}$ are zero-state observable and the dissipation matrices $W \in \mathbb{R}^{q \times q}$ and $W_{c} \in \mathbb{R}^{q \times q}$ are such that there exist, respectively, $\alpha \geq 0, p \in \mathbb{R}_{+}^{q}, \alpha_{\mathrm{c}} \geq 0$, and $p_{\mathrm{c}} \in \mathbb{R}_{+}^{q}$ such that (2.2) is satisfied. Then the following statements hold:

(i) if $\mathscr{G}_{\text {and }} \mathscr{G}_{\mathrm{c}}$ are vector passive and $\tilde{W} \in \mathbb{R}^{q \times q}$ is asymptotically stable, where $\tilde{W}_{(i, j)} \triangleq$ $\max \left\{W_{(i, j)}, W_{\mathrm{c}(i, j)}\right\}, i, j=1, \ldots, q$, then the negative feedback interconnection of $\mathscr{G}$ and $\mathscr{G}_{\mathrm{c}}$ is asymptotically stable;

(ii) if $\mathscr{G}_{\mathrm{S}}$ and $\mathscr{G}_{\mathrm{c}}$ are vector nonexpansive and $\tilde{W} \in \mathbb{R}^{q \times q}$ is asymptotically stable, where $\tilde{W}_{(i, j)} \triangleq \max \left\{W_{(i, j)}, W_{c(i, j)}\right\}, i, j=1, \ldots, q$, then the negative feedback interconnection of $G_{\text {and }} \mathscr{G}_{\mathrm{c}}$ is asymptotically stable.

Proof. The proof is a direct consequence of Theorem 5.1. Specifically, (i) follows from Theorem 5.1 with $R_{\mathrm{c} i}=0, S_{\mathrm{c} i}=I_{m_{\mathrm{c} i}}, Q_{\mathrm{c} i}=0, R_{\mathrm{d} i}=0, S_{\mathrm{d} i}=I_{m_{\mathrm{di}}}, Q_{\mathrm{d} i}=0, R_{\mathrm{cc} i}=0, S_{\mathrm{cc} i}=$ $I_{m_{\mathrm{c} i}}, Q_{\mathrm{cc} i}=0, R_{\mathrm{dc} i}=0, S_{\mathrm{dc} i}=I_{m_{\mathrm{di}}}, Q_{\mathrm{dc} i}=0, i=1, \ldots, q$, and $\Sigma=I_{q}$, while (ii) follows from Theorem 5.1 with $R_{\mathrm{c} i}=\gamma_{\mathrm{ci} i}^{2} I_{m_{\mathrm{c} i}}, S_{\mathrm{c} i}=0, Q_{\mathrm{c} i}=-I_{l_{\mathrm{c} i}}, R_{\mathrm{d} i}=\gamma_{\mathrm{d} i}^{2} I_{m_{\mathrm{d} i}}, S_{\mathrm{d} i}=0, Q_{\mathrm{d} i}=-I_{l_{\mathrm{d} i}}, R_{\mathrm{cc} i}=$ $\gamma_{\mathrm{cc} i}^{2} I_{l_{c i}}, S_{\mathrm{cc} i}=0, Q_{\mathrm{cc} i}=-I_{m_{\mathrm{c} i}}, R_{\mathrm{dc} i}=\gamma_{\mathrm{dc} i}^{2} I_{l_{\mathrm{d} i}}, S_{\mathrm{dc} i}=0, Q_{\mathrm{dc} i}=-I_{m_{\mathrm{d} i}}, i=1, \ldots, q$, and $\Sigma=I_{q}$.

\section{Conclusion}

In this paper, we extended the notion of hybrid dissipativity theory to vector hybrid dissipativity theory. Specifically, using vector storage functions and hybrid supply rates, dissipativity properties of composite large-scale impulsive dynamical systems are shown to be determined from the dissipativity properties of the individual impulsive subsystems and the nature of their interconnections. Furthermore, extended Kalman-Yakubovich-Popov conditions, in terms of the local impulsive subsystem dynamics and the impulsive subsystem interconnection constraints, characterizing vector dissipativeness via vector storage functions, are derived. In addition, general stability criteria were given for feedback interconnections of large-scale impulsive dynamical systems in terms of vector storage functions serving as vector Lyapunov functions. 


\section{Acknowledgment}

This research was supported in part by the Air Force Office of Scientific Research under Grant F49620-03-1-0178 and the National Science Foundation under Grant ECS0133038 .

\section{References}

[1] P. J. Antsaklis and A. Nerode (eds.), Special issue on hybrid control systems, IEEE Trans. Automat. Control 43 (1998), no. 4.

[2] R. Bellman, Vector Lyapunov functions, J. SIAM Control 1 (1962), 32-34.

[3] A. Berman and R. J. Plemmons, Nonnegative Matrices in the Mathematical Sciences, Academic Press (Harcourt Brace Jovanovich Publishers), New York, 1979.

[4] D. S. Bernstein and S. P. Bhat, Nonnegativity, reducibility and semistability of mass action kinetics, Proc. IEEE Conference on Decision and Control (Ariz, 1999), December 1999, pp. $2206-$ 2211.

[5] D. S. Bernstein and D. C. Hyland, Compartmental modeling and second-moment analysis of state space systems, SIAM J. Matrix Anal. Appl. 14 (1993), no. 3, 880-901.

[6] S. Boyd, L. El. Ghaoui, E. Feron, and V. Balakrishnan, Linear Matrix Inequalities in System and Control Theory, SIAM Studies in Applied Mathematics, vol. 15, Society for Industrial and Applied Mathematics (SIAM), Pennsylvania, 1994.

[7] Lj. T. Grujić, A. A. Martynyuk, and M. Ribbens-Pavella, Large Scale Systems Stability under Structural and Singular Perturbations, Lecture Notes in Control and Information Sciences, vol. 92, Springer-Verlag, Berlin, 1987.

[8] W. M. Haddad and V. Chellaboina, Dissipativity theory and stability of feedback interconnections for hybrid dynamical systems, Math. Probl. Eng. 7 (2001), no. 4, 299-335.

[9] W. M. Haddad, V. Chellaboina, and E. August, Stability and dissipativity theory for nonnegative dynamical systems: a thermodynamic framework for biological and physiological systems, Proc. IEEE Conference on Decision and Control (Fla, 2001), December 2001, pp. 442-458.

[10] W. M. Haddad, V. Chellaboina, and N. A. Kablar, Non-linear impulsive dynamical systems. I. Stability and dissipativity, Internat. J. Control 74 (2001), no. 17, 1631-1658.

[11] _ Non-linear impulsive dynamical systems. II. Stability of feedback interconnections and optimality, Internat. J. Control 74 (2001), no. 17, 1659-1677.

[12] W. M. Haddad, V. Chellaboina, and S. G. Nersesov, Large-scale nonlinear dynamical systems: a vector dissipative systems approach, Proc. IEEE Conference on Decision and Control (Hawaii, 2003), December 2003, pp. 5603-5608.

[13] _ Thermodynamics and large-scale nonlinear dynamical systems: a vector dissipative systems approach, Dynam. Contin. Discrete Impuls. Systems 11 (2004), 609-649.

[14] D. J. Hill and P. J. Moylan, Stability results for nonlinear feedback systems, Automatica J. IFAC 13 (1977), no. 4, 377-382.

[15] V. Lakshmikantham, D. D. Baĭnov, and P. S. Simeonov, Theory of Impulsive Differential Equations, Series in Modern Applied Mathematics, vol. 6, World Scientific Publishing, New Jersey, 1989.

[16] V. Lakshmikantham, V. M. Matrosov, and S. Sivasundaram, Vector Lyapunov Functions and Stability Analysis of Nonlinear Systems, Mathematics and Its Applications, vol. 63, Kluwer Academic Publishers, Dordrecht, 1991.

[17] J. Lunze, Stability analysis of large-scale systems composed of strongly coupled similar subsystems, Automatica J. IFAC 25 (1989), no. 4, 561-570.

[18] A. A. Martynyuk, Stability of Motion of Complex Systems, Izdat. Naukova Dumka, Kiev, 1975. 
262 Vector dissipativity and large-scale impulsive systems

[19] Stability by Liapunov's Matrix Function Method with Applications, Monographs and Textbooks in Pure and Applied Mathematics, vol. 214, Marcel Dekker, New York, 1998.

[20] Q Qualitative Methods in Nonlinear Dynamics. Novel Approaches to Liapunov's Matrix Functions, Monographs and Textbooks in Pure and Applied Mathematics, vol. 246, Marcel Dekker, New York, 2002.

[21] V. M. Matrosov, Method of Lyapunov-vector functions in feedback systems, Avtomat. i Telemeh. (1972), no. 9, 63-75 (Russian).

[22] A. N. Michel and B. Hu, Towards a stability theory of general hybrid dynamical systems, Automatica J. IFAC 35 (1999), no. 3, 371-384.

[23] A. N. Michel and R. K. Miller, Qualitative Analysis of Large Scale Dynamical Systems, Academic Press (Harcourt Brace Jovanovich Publishers), New York, 1977.

[24] A. N. Michel, K. Wang, and B. Hu, Qualitative Theory of Dynamical Systems. The Role of Stability Preserving Mappings, Monographs and Textbooks in Pure and Applied Mathematics, vol. 239, Marcel Dekker, New York, 2001.

[25] A. S. Morse, C. C. Pantelides, S. Sastry, and J. M. Schumacher (eds.), Special issue on hybrid control systems, Automatica J. IFAC 35 (1999), no. 3.

[26] D. D. Šiljak, Large-Scale Dynamic Systems. Stability and Structure, North-Holland Series in System Science and Engineering, vol. 3, North-Holland, New York, 1979.

[27] Complex dynamic systems: dimensionality, structure, and uncertainty, Large Scale Systems 4 (1983), 279-294.

[28] H. Ye, A. N. Michel, and L. Hou, Stability theory for hybrid dynamical systems, IEEE Trans. Automat. Control 43 (1998), no. 4, 461-474.

Wassim M. Haddad: School of Aerospace Engineering, Georgia Institute of Technology, Atlanta, GA 30332-0150, USA

E-mail address:wm.haddad@aerospace.gatech.edu

VijaySekhar Chellaboina: Mechanical and Aerospace Engineering, University of MissouriColumbia, Columbia, MO 65211, USA

E-mail address: chellaboinav@missouri.edu

Qing Hui: School of Aerospace Engineering, Georgia Institute of Technology, Atlanta, GA 303320150, USA

E-mail address: qing_hui@ae.gatech.edu

Sergey Nersesov: School of Aerospace Engineering, Georgia Institute of Technology, Atlanta, GA 30332-0150, USA

E-mail address: sergei_nersesov@ae.gatech.edu 


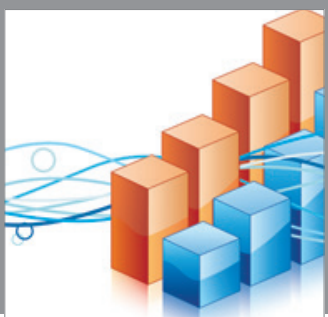

Advances in

Operations Research

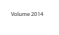

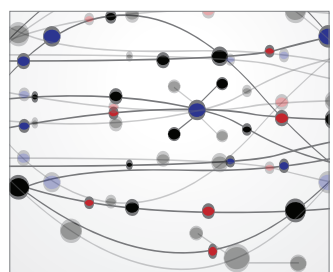

\section{The Scientific} World Journal
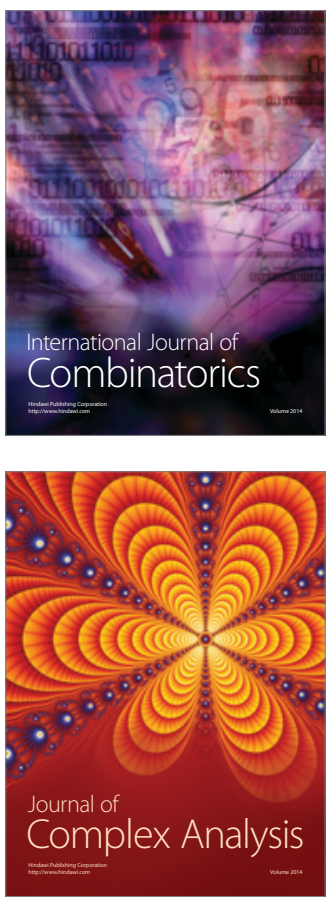

International Journal of

Mathematics and

Mathematical

Sciences
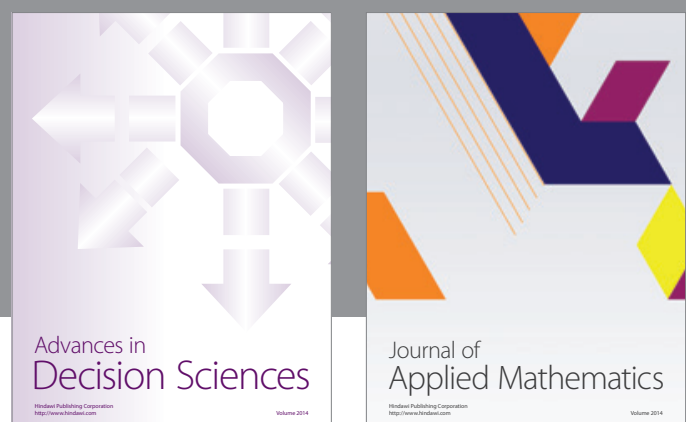

Journal of

Applied Mathematics
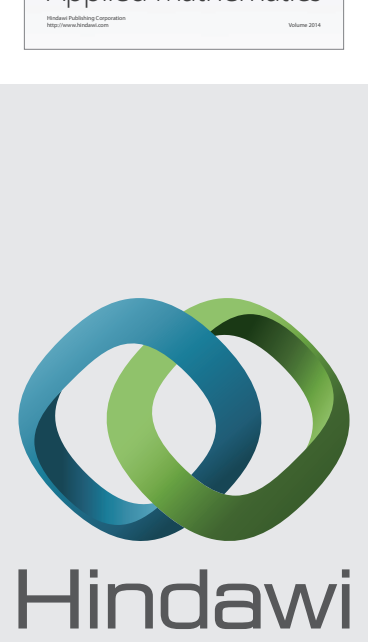

Submit your manuscripts at http://www.hindawi.com
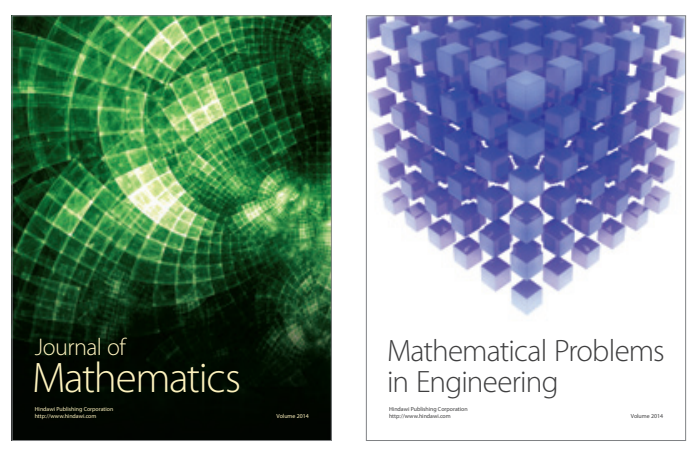

Mathematical Problems in Engineering
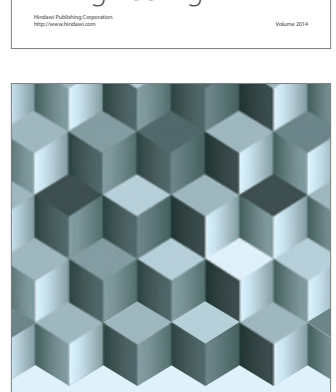

Journal of

Function Spaces
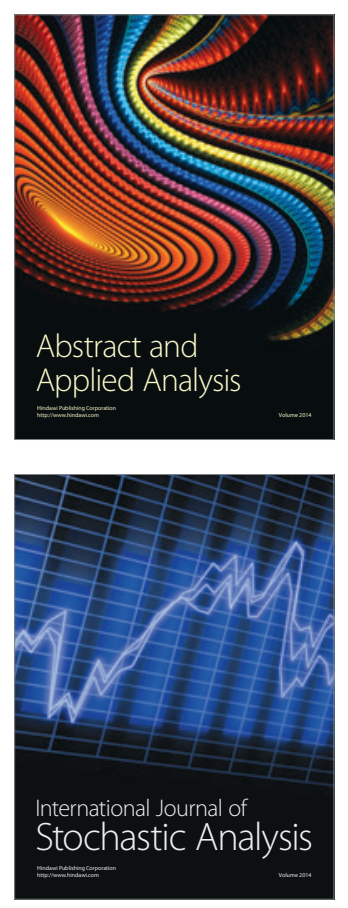

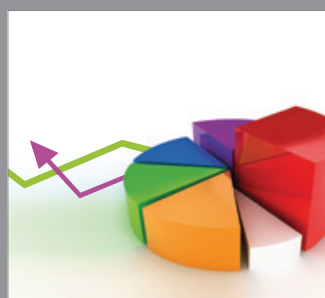

ournal of

Probability and Statistics

Promensencen
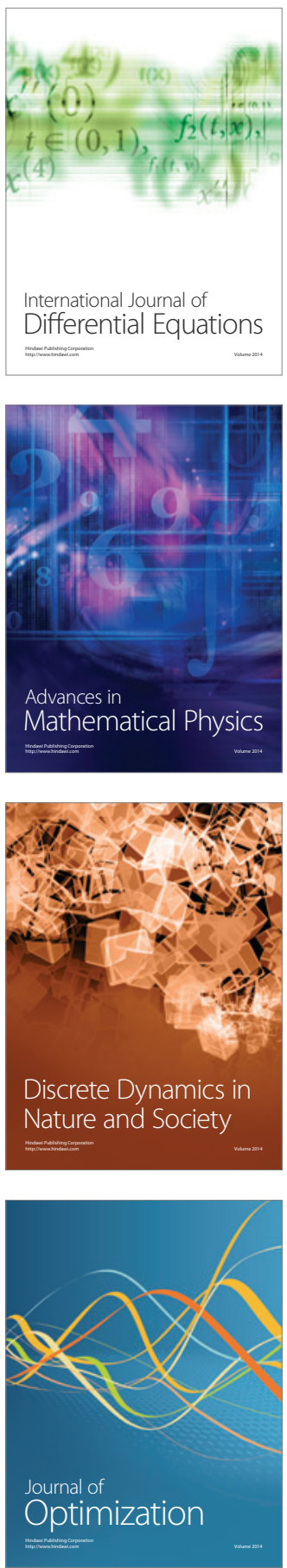